ESAIM: M2AN 55 (2021) 833-885

https://doi.org/10.1051/m2an/2021014
ESAIM: Mathematical Modelling and Numerical Analysis

www.esaim-m2an.org

\title{
STABLE APPROXIMATIONS FOR AXISYMMETRIC WILLMORE FLOW FOR CLOSED AND OPEN SURFACES *
}

\author{
John W. Barrett ${ }^{1}$, Harald Garcke $^{2}$ and Robert NÜRnBerg ${ }^{3, *}$
}

\begin{abstract}
For a hypersurface in $\mathbb{R}^{3}$, Willmore flow is defined as the $L^{2}$-gradient flow of the classical Willmore energy: the integral of the squared mean curvature. This geometric evolution law is of interest in differential geometry, image reconstruction and mathematical biology. In this paper, we propose novel numerical approximations for the Willmore flow of axisymmetric hypersurfaces. For the semidiscrete continuous-in-time variants we prove a stability result. We consider both closed surfaces, and surfaces with a boundary. In the latter case, we carefully derive weak formulations of suitable boundary conditions. Furthermore, we consider many generalizations of the classical Willmore energy, particularly those that play a role in the study of biomembranes. In the generalized models we include spontaneous curvature and area difference elasticity (ADE) effects, Gaussian curvature and line energy contributions. Several numerical experiments demonstrate the efficiency and robustness of our developed numerical methods.
\end{abstract}

Mathematics Subject Classification. 65M60, 65M12, 35K55, 53C44.

Received November 4, 2019. Accepted March 12, 2021.

\section{INTRODUCTION}

Geometric functionals involving the principal curvatures of a two-dimensional surface play an important role in mechanics, geometry, imaging and biology. In plate and shell theories such functionals go back to the work of $[22,26,31]$. In geometry, an energy given by the integrated square of the mean curvature has been studied intensively since the pioneering work of [36]. Especially variational problems are of interest and the famous Willmore conjecture, which states that the minimizer among genus 1 surfaces is given by the Clifford torus, was only solved recently by [28]. In imaging, boundary value problems involving the Willmore functional have been used in problems related to image inpainting and surface restoration, see [12,15]. In the theory of biological membranes and vesicles, side constraints on surface area and enclosed volume, and more general curvature functionals play a role. In a work of [13] a possible explanation of the shape of the human red blood was given

Keywords and phrases. Willmore flow, Helfrich flow, axisymmetry, parametric finite elements, stability, tangential movement, spontaneous curvature, ADE model, clamped boundary conditions, Navier boundary conditions, Gaussian curvature energy, line energy.

* John died on 30 June 2019, when this manuscript was nearly completed. We dedicate this article to his memory.

1 Department of Mathematics, Imperial College, London SW7 2AZ, UK.

2 Fakultät für Mathematik, Universität Regensburg, 93040 Regensburg, Germany.

3 Department of Mathematics, University of Trento, Trento, Italy.

* Corresponding author: robert.nurnberg@unitn.it 
using a curvature functional together with volume and area constraints. In this approach the membrane is modeled as a two-dimensional surface. Later [23], in a seminal paper, introduced the energy

$$
\frac{\alpha}{2} \int_{\mathcal{S}}\left(k_{m}-\bar{\varkappa}\right)^{2} \mathrm{~d} \mathcal{H}^{2}+\alpha_{G} \int_{\mathcal{S}} k_{g} \mathrm{~d} \mathcal{H}^{2}
$$

for a surface $\mathcal{S}$ in $\mathbb{R}^{3}$, where $k_{m}$ is the mean curvature, $k_{g}$ is the Gaussian curvature, d $\mathcal{H}^{2}$ stands for integration with respect to the two-dimensional surface measure and $\alpha, \alpha_{G}$ are so-called bending rigidities. The important new ingredient is the term $\bar{\varkappa}$, the so-called spontaneous curvature, which reflects a possible asymmetry in the membrane. In biological applications, membranes in equilibrium minimize (1.1) under volume and area constraints on the surface $\mathcal{S}$.

The simplest evolution law which decreases the energy (1.1), and which can be used to obtain minimizers, is the $L^{2}$-gradient flow

$$
\mathcal{V}_{\mathcal{S}}=-\alpha \Delta_{\mathcal{S}} k_{m}+2 \alpha\left(k_{m}-\bar{\varkappa}\right) k_{g}-\frac{\alpha}{2}\left(k_{m}^{2}-\bar{\varkappa}^{2}\right) k_{m},
$$

where $\mathcal{V}_{\mathcal{S}}$ is the normal velocity of an evolving surface $(\mathcal{S}(t))_{t \in[0, T]}$. The above formula shows that the Gauss curvature term $\alpha_{G} \int_{\mathcal{S}} k_{g} \mathrm{~d} \mathcal{H}^{2}$ does not give a contribution to the flow, which is due to the fact that for closed surfaces $\int_{\mathcal{S}} k_{g} \mathrm{~d} \mathcal{H}^{2}$ is a topological invariant. For surfaces with boundary, however, the term $\int_{\mathcal{S}} k_{g} \mathrm{~d} \mathcal{H}^{2}$ enters the $L^{2}$-gradient flow via boundary conditions. The equation (1.2) also shows that the $L^{2}$-gradient flow is highly nonlinear and for open surfaces also highly nonlinear boundary conditions have to be considered.

It is the goal of this paper to introduce variational discretizations for an axisymmetric formulation of (1.2), and to show stability estimates in a semi-discrete setting. The main contributions of this paper are as follows.

- Using a Lagrangian calculus we derive two mixed formulations of (1.2), which can be used for all boundary conditions which appear in geometry and applications.

- The derivation of continuous-in-time, discrete-in-space formulations for which stability bounds can be shown.

- A proof of an equidistribution property for one of the schemes, which relies on an implicit tangential motion of vertices and leads to a uniform distribution of vertices on the polygonal curve everywhere where the curve is not locally flat. We refer to our review article [11] for more information on the background of this tangential motion.

- For fully discrete variants, existence and uniqueness results are shown under mild assumptions.

- Numerical computations show the efficiency of the approach.

To our knowledge, this is the first time that weak formulations involving general boundary conditions are derived in the axisymmetric setting.

To describe earlier literature in more detail let us discuss the geometry under consideration. We consider the case that $\mathcal{S}(t)$ is an axisymmetric surface, which is rotationally symmetric with respect to the $x_{2}$-axis, see Figure 1. Besides the geometry of a closed surface, we allow for open surfaces, i.e. the boundary of the rotationally symmetric surface can consist of either one or two circles. Altogether four different topologies can be considered: surfaces of spherical topology, surfaces of toroidal topology, surfaces suspended between two rings and surfaces suspended at one ring. We have to compute the evolution of a curve which then has to be rotated around the $x_{2}$-axis. For boundary points on the $x_{2}$-axis, singular and degenerate behaviour in the resulting equations appear, which makes the analytical and numerical treatment difficult. At other boundary points, which correspond to a boundary ring of the surface $\mathcal{S}(t)$, one has to describe further conditions, which can be of the following form:

- Clamped boundary conditions (position and angle fixed at the boundary).

- Navier boundary conditions (position fixed and a natural boundary condition involving the mean curvature).

- Semifree boundary conditions, i.e. the boundary is free to move on a plane.

- Free boundary conditions, for which several natural boundary conditions have to hold.

The motivation behind considering axisymmetric geometries is clear: a vastly more efficient numerical method, compared to truly three-dimensional computations. On the other hand, many situations of interest in practice 

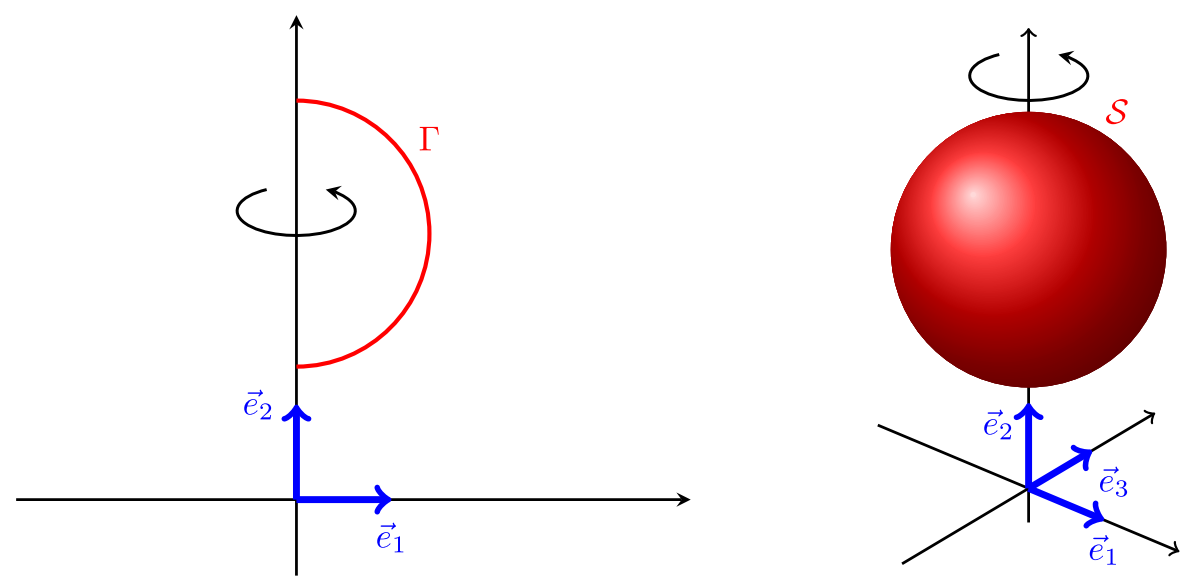

Figure 1 . Sketch of $\Gamma$ and $\mathcal{S}$, as well as the unit vectors $\vec{e}_{1}, \vec{e}_{2}$ and $\vec{e}_{3}$.

do have rotational symmetry. Moreover, some qualitative aspects of the considered evolution equations, or the impact of certain physical parameters, can often be studied in the axisymmetric setting. For example, in Figure 24 of [7], we numerically studied the onset of a singularity for Willmore flow for a surface of genus 0 , and we perform an analogous investigation for genus- 1 surfaces in Appendix B of this paper. The axisymmetric setting is hence very popular in the (bio-)physics literature and we refer to $[14,25,34]$ for a derivation of the equilibrium equations for axisymmetric open membranes.

Earlier results on the numerical approximation of geometric evolution problems in the axisymmetric setting can be found in $[7,9]$, as well as on the surprisingly closely related problem of curve evolutions in Riemannian manifolds, see $[8,10]$. There appears to be little numerical analysis for such evolution problems in the literature. In the case of Willmore flow, we refer to $[7,16,20,29]$ for existing numerical approaches. The present paper fills the gap left by [7], where two schemes for axisymmetric Willmore flow of closed surfaces were considered, for which no stability proofs appear to exist. The numerical analysis will share certain features with our earlier works [3$6,8]$, work that has been critically influenced and inspired by the seminal works [19,21]. However, as mentioned already above, the axisymmetry introduces additional difficulties due to degenerate or singular coefficients that have to be taken care of in the analysis and in the numerical treatment. For numerical approaches of the flow (1.2) for open membranes without the restriction of axisymmetry, we refer to [6,35], where the latter authors use a phase field approach.

The rest of the paper is organized as follows. In Section 2 we postulate the mathematical problems we would like to consider, and in Section 3 we state suitable weak formulations for the evolution problems. Based on these weak formulations we introduce two types of semidiscrete schemes in Section 4 and prove their stability. We also consider approximations for the area and volume preserving variants of Willmore flow. The corresponding fully discrete schemes are presented in Section 5, and numerical results are shown in Section 6. Finally, in Appendix A we prove the consistency of the weak formulations introduced in Section 3, including the considered boundary conditions, while in Appendix B we present numerical evidence for the onset of a singularity for Willmore flow of genus-1 surfaces.

\section{Mathematical formulation}

\subsection{Generating curve}

Let $\mathbb{R} / \mathbb{Z}$ be the periodic interval $[0,1]$, and set

$$
I=\mathbb{R} / \mathbb{Z}, \text { with } \partial I=\emptyset, \quad \text { or } \quad I=(0,1), \text { with } \partial I=\{0,1\} .
$$


We consider the axisymmetric situation, where $\vec{x}(\cdot, t): \bar{I} \rightarrow \mathbb{R}_{\geq 0} \times \mathbb{R}$ is a parameterization of $\Gamma(t)$. Throughout $\Gamma(t)$ represents the generating curve of a surface $\mathcal{S}(t)$ that is axisymmetric with respect to the $x_{2}$-axis, see Figure 1. In particular, on defining $\vec{\Pi}_{3}^{3}(r, z, \theta)=(r \cos \theta, z, r \sin \theta)^{T}$ for $r \in \mathbb{R}_{\geq 0}, z \in \mathbb{R}, \theta \in[0,2 \pi]$ and $\Pi_{2}^{3}(r, z)=\left\{\vec{\Pi}_{3}^{3}(r, z, \theta): \theta \in[0,2 \pi)\right\}$, we have that

$$
\mathcal{S}(t)=\bigcup_{(r, z)^{T} \in \Gamma(t)} \Pi_{2}^{3}(r, z)=\bigcup_{\rho \in \bar{I}} \Pi_{2}^{3}(\vec{x}(\rho, t)) .
$$

Here we allow $\Gamma(t)$ to be either a closed curve, parameterized over $\mathbb{R} / \mathbb{Z}$, which corresponds to $\mathcal{S}(t)$ being a genus-1 surface without boundary. Or $\Gamma(t)$ may be an open curve, parameterized over $[0,1]$. If both ends of $\Gamma(t)$ are attached to the $x_{2}$-axis, then $\mathcal{S}(t)$ is a genus- 0 surface without boundary. If only one end of $\Gamma(t)$ is attached to the $x_{2}$-axis, then $\mathcal{S}(t)$ is an open surface with boundary, where the boundary consists of a single connected component. If no endpoint of $\Gamma(t)$ is attached to the $x_{2}$-axis, then $\mathcal{S}(t)$ is an open surface with boundary, where the boundary consists of two connected components. On the boundary we either prescribe clamped boundary conditions, or Navier boundary conditions, or semifree boundary conditions, or free boundary conditions. For clamped and Navier boundary conditions, the boundary point is fixed in space, while for the semifree boundary conditions the boundary point is allowed to move on a line parallel to one of the two axes. As the name for the free boundary condition suggests, the endpoint is free to move in space. In order to define the different boundary conditions, we let $\partial_{0} I \cup \partial_{C} I \cup \partial_{N} I \cup \partial_{1} I \cup \partial_{2} I \cup \partial_{F} I$ be a disjoint partitioning of $\partial I$, with $\partial_{0} I$ denoting the subset of boundary points of $I$ that correspond to endpoints of $\Gamma(t)$ attached to the $x_{2}$-axis. Moreover, $\partial_{C} I, \partial_{N} I, \partial_{S F} I=\partial_{1} I \cup \partial_{2} I$ and $\partial_{F} I$ correspond to clamped, Navier, semifree and free boundary conditions, respectively. See Table 1 for a visualization of the different types of boundary nodes.

Hence, we always assume that, for all $t \in[0, T]$,

$$
\begin{aligned}
\vec{x}(\rho, t) \cdot \vec{e}_{1} & >0 & & \forall \rho \in \bar{I} \backslash \partial_{0} I, \\
\vec{x}(\rho, t) \cdot \vec{e}_{1} & =0 & & \forall \rho \in \partial_{0} I, \\
\vec{x}_{t}(\rho, t) & =\overrightarrow{0} & & \forall \rho \in \partial_{C} I \cup \partial_{N} I, \\
\vec{x}_{t}(\rho, t) \cdot \vec{e}_{i} & =0 & & \forall \rho \in \partial_{i} I, i=1,2 .
\end{aligned}
$$

We will discuss the additional boundary conditions to $(2.2 \mathrm{~b})-(2.2 \mathrm{~d})$ later in this section.

On assuming that $\left|\vec{x}_{\rho}\right| \geq c_{0}>0$ in $\bar{I} \times[0, T]$, we introduce the arclength $s$ of the curve, i.e. $\partial_{s}=\left|\vec{x}_{\rho}\right|^{-1} \partial_{\rho}$, and set

$$
\vec{\tau}(\rho, t)=\vec{x}_{s}(\rho, t)=\frac{\vec{x}_{\rho}(\rho, t)}{\left|\vec{x}_{\rho}(\rho, t)\right|} \quad \text { and } \quad \vec{\nu}(\rho, t)=-[\vec{\tau}(\rho, t)]^{\perp} \quad \text { in } \bar{I},
$$

where $(\cdot)^{\perp}$ denotes a clockwise rotation by $\frac{\pi}{2}$.

On recalling (2.1), we observe that the normal $\vec{n}_{\mathcal{S}}$ on $\mathcal{S}(t)$ is given by

$$
\vec{n}_{\mathcal{S}}\left(\vec{\Pi}_{3}^{3}(\vec{x}(\rho, t), \theta)\right)=\left(\begin{array}{c}
\left(\vec{\nu}(\rho, t) \cdot \vec{e}_{1}\right) \cos \theta \\
\vec{\nu}(\rho, t) \cdot \vec{e}_{2} \\
\left(\vec{\nu}(\rho, t) \cdot \vec{e}_{1}\right) \sin \theta
\end{array}\right) \quad \text { for } \quad \rho \in \bar{I}, t \in[0, T], \theta \in[0,2 \pi) .
$$

Similarly, the normal velocity $\mathcal{V}_{\mathcal{S}}$ of $\mathcal{S}(t)$ in the direction $\vec{n}_{\mathcal{S}}$ is given by

$$
\mathcal{V}_{\mathcal{S}}=\vec{x}_{t}(\rho, t) \cdot \vec{\nu}(\rho, t) \quad \text { on } \Pi_{2}^{3}(\vec{x}(\rho, t)) \subset \mathcal{S}(t), \quad \forall \rho \in \bar{I}, t \in[0, T] .
$$

For the curvature $\varkappa$ of $\Gamma(t)$ it holds that

$$
\varkappa \vec{\nu}=\vec{\varkappa}=\vec{\tau}_{s}=\frac{1}{\left|\vec{x}_{\rho}\right|}\left[\frac{\vec{x}_{\rho}}{\left|\vec{x}_{\rho}\right|}\right]_{\rho} \text { in } \bar{I} .
$$


TABLE 1 . The different types of boundary nodes enforced by $(2.2 \mathrm{~b})-(2.2 \mathrm{~d})$, and their effect on the possible movement of the boundary circles $\partial \mathcal{S}$. Here the boundary circles in $\mathbb{R}^{3}$ are shown with the help of an oblique projection.

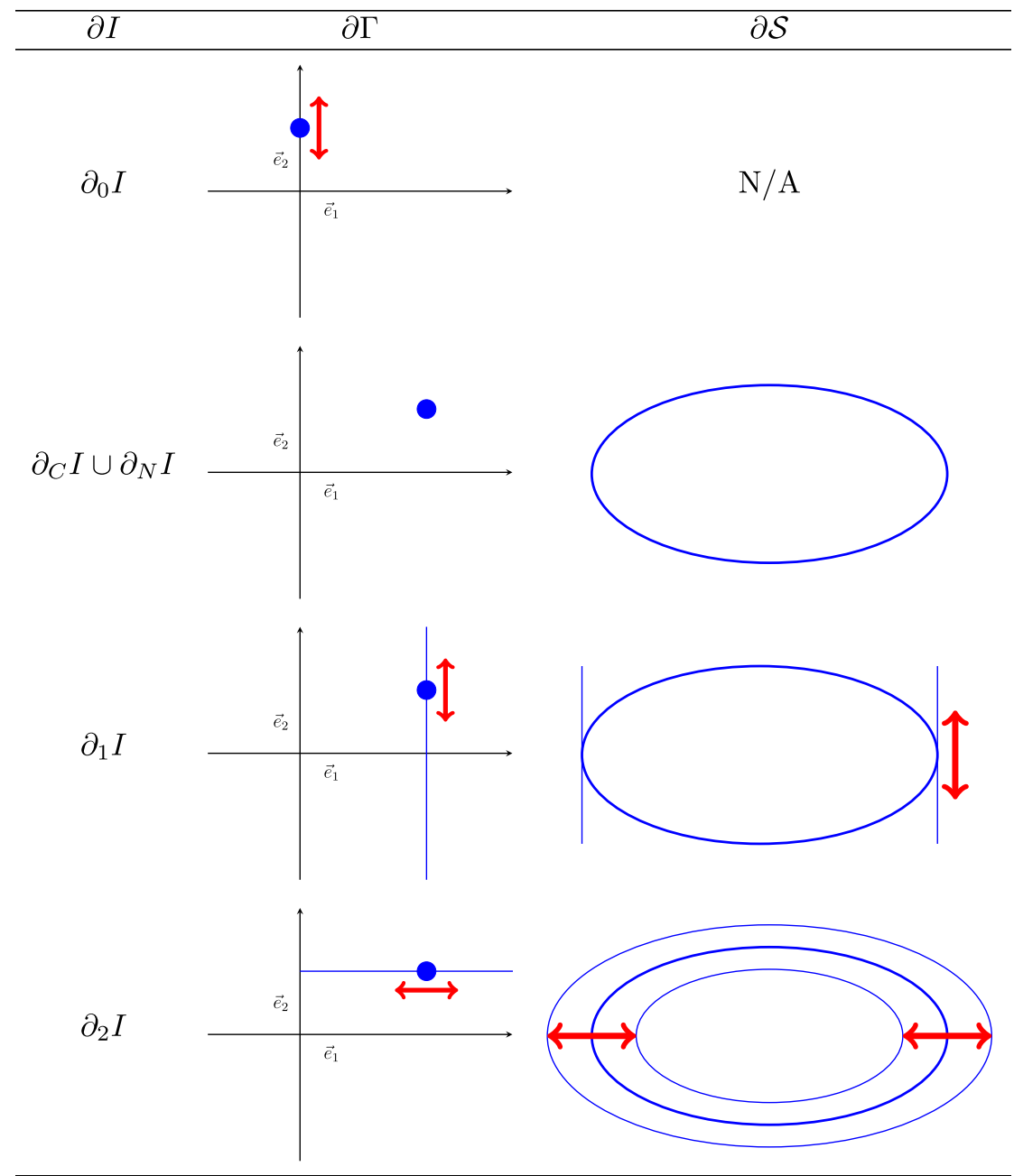

We recall that the so-called mean curvature, i.e. the sum of the principal curvatures, and Gaussian curvature of $\mathcal{S}(t)$ are given by

$$
\varkappa_{\mathcal{S}}=\varkappa-\frac{\vec{\nu} \cdot \vec{e}_{1}}{\vec{x} \cdot \vec{e}_{1}} \quad \text { and } \quad \mathcal{K}_{\mathcal{S}}=-\varkappa \frac{\vec{\nu} \cdot \vec{e}_{1}}{\vec{x} \cdot \vec{e}_{1}}=\varkappa\left(\varkappa_{\mathcal{S}}-\varkappa\right) \text { in } \bar{I},
$$

respectively; see e.g. (2.11) of [7]. More precisely, if $k_{m}$ and $k_{g}$ denote the mean and Gaussian curvatures of $\mathcal{S}(t)$, then

$$
k_{m}=\varkappa_{\mathcal{S}}(\rho, t) \text { and } k_{g}=\mathcal{K}_{\mathcal{S}}(\rho, t) \quad \text { on } \Pi_{2}^{3}(\vec{x}(\rho, t)) \subset \mathcal{S}(t), \quad \forall \rho \in \bar{I}, t \in[0, T] .
$$

In the literature, the two terms making up $\varkappa_{\mathcal{S}}$ in (2.7) are often referred to as in-plane and azimuthal curvatures, respectively, with their sum being equal to the mean curvature. We note that combining (2.7) and (2.6) yields 
that

$$
\varkappa_{\mathcal{S}} \vec{\nu}=\frac{1}{\left|\vec{x}_{\rho}\right|}\left[\frac{\vec{x}_{\rho}}{\left|\vec{x}_{\rho}\right|}\right]_{\rho}-\frac{\vec{\nu} \cdot \vec{e}_{1}}{\vec{x} \cdot \vec{e}_{1}} \vec{\nu} \text { in } \bar{I} .
$$

Weak formulations of (2.6) and (2.9) will form the basis of our approximations for Willmore flow. Clearly, for a smooth surface with bounded curvatures it follows from (2.7) that

$$
\vec{\nu}(\rho, t) \cdot \vec{e}_{1}=0 \quad \forall \rho \in \partial_{0} I, t \in[0, T],
$$

which is clearly equivalent to

$$
\vec{x}_{\rho}(\rho, t) \cdot \vec{e}_{2}=0 \quad \forall \rho \in \partial_{0} I, t \in[0, T] .
$$

A precise derivation of (2.11) in the context of a weak formulation of (2.9) can be found in Appendix A of [9]. We note that for the singular fraction in (2.7) it follows from (2.11) and (2.10), on recalling (2.6), that

$$
\lim _{\rho \rightarrow \rho_{0}} \frac{\vec{\nu}(\rho, t) \cdot \vec{e}_{1}}{\vec{x}(\rho, t) \cdot \vec{e}_{1}}=\lim _{\rho \rightarrow \rho_{0}} \frac{\vec{\nu}_{\rho}(\rho, t) \cdot \vec{e}_{1}}{\vec{x}_{\rho}(\rho, t) \cdot \vec{e}_{1}}=\vec{\nu}_{s}\left(\rho_{0}, t\right) \cdot \vec{\tau}\left(\rho_{0}, t\right)=-\varkappa\left(\rho_{0}, t\right) \quad \forall \rho_{0} \in \partial_{0} I, t \in[0, T] .
$$

\subsection{Willmore flow}

We now define the generalized Willmore energy of the surface $\mathcal{S}(t)$ as

$$
E(t)=\frac{1}{2} \alpha \int_{\mathcal{S}(t)}\left(k_{m}-\bar{\varkappa}\right)^{2} \mathrm{~d} \mathcal{H}^{2}=\pi \alpha \int_{I} \vec{x} \cdot \vec{e}_{1}\left(\varkappa_{\mathcal{S}}-\bar{\varkappa}\right)^{2}\left|\vec{x}_{\rho}\right| \mathrm{d} \rho,
$$

where we have recalled (2.8); see also (6) and (7) of [16]. Here $\alpha \in \mathbb{R}_{>0}$ and $\bar{\varkappa} \in \mathbb{R}$ are given constants, with $\bar{\varkappa}$ denoting the so-called spontaneous curvature. On $\mathcal{S}(t)$, Willmore flow, i.e. the $L^{2}$-gradient flow for (2.13), is given by

$$
\frac{1}{\alpha} \mathcal{V}_{\mathcal{S}}=-\Delta_{\mathcal{S}} k_{m}+2\left(k_{m}-\bar{\varkappa}\right) k_{g}-\frac{1}{2}\left(k_{m}^{2}-\bar{\varkappa}^{2}\right) k_{m} \quad \text { on } \mathcal{S}(t),
$$

recall (2.5) for the definition of $\mathcal{V}_{\mathcal{S}}$, see e.g. [2]. Here $\Delta_{\mathcal{S}}=\nabla_{\mathcal{S}} \cdot \nabla_{\mathcal{S}}$ is the Laplace-Beltrami operator on $\mathcal{S}(t)$. Associated with (2.14) are boundary conditions, but we will discuss these once we have generalized the energy (2.13).

For applications to biomembranes, and for surfaces with boundary, the considered energy can be more general than (2.13). In particular, surface area constraints, area difference elasticity (ADE) effects, Gaussian curvature contributions and line energy now also play a role. Hence, we adapt (2.13) to

$$
E(t)=\frac{1}{2} \alpha \int_{\mathcal{S}(t)}\left(k_{m}-\bar{\varkappa}\right)^{2} \mathrm{~d} \mathcal{H}^{2}+\lambda \mathcal{H}^{2}(\mathcal{S}(t))+\frac{\beta}{2} \mathcal{A}_{\mathcal{S}}^{2}(t)+\alpha_{G} \int_{\mathcal{S}(t)} k_{g} \mathrm{~d} \mathcal{H}^{2}+\varsigma \mathcal{H}^{1}(\partial \mathcal{S}(t))
$$

with $\mathcal{A}_{\mathcal{S}}(t)=\int_{\mathcal{S}(t)} k_{m} \mathrm{~d} \mathcal{H}^{2}-M_{0}$, and given constants $\beta \in \mathbb{R}_{\geq 0}, M_{0}, \alpha_{G}, \varsigma \in \mathbb{R}$, see e.g. $[5,6,13,14,23,24,30,33,34]$ for more details. In addition, $\lambda \in \mathbb{R}$ is a constant that can penalise or encourage surface area growth. If chosen time-dependent, it can act as a Lagrange multiplier for a surface area constraint. We make this more explicit later on, see Section 2.4 below. We remark that the contributions $\frac{1}{2} \alpha \int_{\mathcal{S}(t)} k_{m}^{2} \mathrm{~d} \mathcal{H}^{2}+\alpha_{G} \int_{\mathcal{S}(t)} k_{g} \mathrm{~d} \mathcal{H}^{2}$ to the energy $E(t)$ are positive semidefinite with respect to the principal curvatures only if $\alpha_{G} \in[-2 \alpha, 0]$. We note that this constraint is likely to have implications for the existence and regularity of the corresponding $L^{2}$-gradient flow.

Noting the Gauss-Bonnet theorem, see [27],

$$
\int_{\mathcal{S}} k_{g} \mathrm{~d} \mathcal{H}^{2}=2 \pi m(\mathcal{S})+\int_{\partial \mathcal{S}} k_{\partial \mathcal{S}, \mu} \mathrm{d} \mathcal{H}^{1},
$$


where $m(\mathcal{S}) \in \mathbb{Z}$ denotes the Euler characteristic of $\mathcal{S}$ and $k_{\partial \mathcal{S}, \mu}$ is the geodesic curvature of $\partial \mathcal{S}$, we can rewrite $(2.15)$ as

$$
E(t)=\frac{1}{2} \alpha \int_{\mathcal{S}(t)}\left(k_{m}-\bar{\varkappa}\right)^{2} \mathrm{~d} \mathcal{H}^{2}+\lambda \mathcal{H}^{2}(\mathcal{S}(t))+\frac{\beta}{2} \mathcal{A}_{\mathcal{S}}^{2}(t)+\alpha_{G}\left[\int_{\partial \mathcal{S}(t)} k_{\partial \mathcal{S}, \mu} \mathrm{d} \mathcal{H}^{1}+2 \pi m(\mathcal{S}(t))\right]+\varsigma \mathcal{H}^{1}(\partial \mathcal{S}(t)) .
$$

In order to define $k_{\partial \mathcal{S}, \mu}$, we first define the conormal, $\vec{\mu}_{\partial \mathcal{S}}$, to $\mathcal{S}(t)$ on $\partial \mathcal{S}(t)$ to be

$$
\vec{\mu}_{\partial \mathcal{S}}= \pm \vec{n}_{\mathcal{S}} \times \overrightarrow{\mathrm{id}}_{s} \quad \text { on } \partial \mathcal{S}(t)
$$

where id denotes the identity in $\mathbb{R}^{3}$ and $s$ denotes arclength on the curve $\partial \mathcal{S}(t) \subset \mathbb{R}^{3}$, so that $\overrightarrow{\mathrm{d}}_{s}$ is its unit tangent vector. The sign in (2.18) is chosen so that $\vec{\mu}_{\partial \mathcal{S}}$ points out of $\mathcal{S}(t)$. It holds that $\overrightarrow{\mathrm{id}}_{s s}=\vec{k}_{\partial \mathcal{S}}=k_{\partial \mathcal{S}, n} \vec{n}_{\mathcal{S}}+k_{\partial \mathcal{S}, \mu} \vec{\mu}_{\partial \mathcal{S}}$ on $\partial \mathcal{S}(t)$, where $\vec{k}_{\partial \mathcal{S}}$ is the curvature vector on $\partial \mathcal{S}(t)$, and where $k_{\partial \mathcal{S}, n}$ is the normal curvature and $k_{\partial \mathcal{S}, \mu}$ is the geodesic curvature of $\partial \mathcal{S}(t)$.

Similarly to $(2.8)$, it is easily seen that

$$
k_{\partial \mathcal{S}, n}=-\frac{\vec{\nu}(\rho, t) \cdot \vec{e}_{1}}{\vec{x}(\rho, t) \cdot \vec{e}_{1}} \text { and } k_{\partial \mathcal{S}, \mu}=-\frac{\vec{\mu}(\rho, t) \cdot \vec{e}_{1}}{\vec{x}(\rho, t) \cdot \vec{e}_{1}} \quad \text { on } \Pi_{2}^{3}(\vec{x}(\rho, t)) \subset \partial \mathcal{S}(t), \quad \forall \rho \in \partial I \backslash \partial_{0} I, t \in[0, T],
$$

where $\vec{\nu}(\cdot, t)$ is the unit normal on $\Gamma(t)$ as defined in (2.3) and (2.4) and

$$
\vec{\mu}(p, t)=(-1)^{p+1} \vec{\tau}(p, t) \quad \forall p \in \partial I, t \in[0, T],
$$

denotes the corresponding conormal of $\Gamma(t)$ at the endpoint $\vec{x}(p, t)$, for $p \in \partial I$. Here, we have recalled that the conormal $\vec{\mu}_{\partial \mathcal{S}}$ points out of $\mathcal{S}(t)$.

Hence, an energy equivalent to (2.17), for flows of axisymmetric surfaces without topological changes, can be written as

$$
\begin{aligned}
\widetilde{E}(t)= & E(t)-2 \pi \alpha_{G} m(\mathcal{S}(t)) \\
= & \pi \alpha \int_{I} \vec{x} \cdot \vec{e}_{1}\left[\varkappa_{\mathcal{S}}-\bar{\varkappa}\right]^{2}\left|\vec{x}_{\rho}\right| \mathrm{d} \rho+2 \pi \lambda \int_{I}\left(\vec{x} \cdot \vec{e}_{1}\right)\left|\vec{x}_{\rho}\right| \mathrm{d} \rho+\frac{\beta}{2} \mathcal{A}_{\mathcal{S}}^{2}(t) \\
& -2 \pi \alpha_{G} \sum_{p \in \partial I \backslash \partial_{0} I}\left[\vec{x} \cdot \vec{e}_{1} \frac{\vec{\mu} \cdot \vec{e}_{1}}{\vec{x} \cdot \vec{e}_{1}}\right](p)+2 \pi \varsigma \sum_{p \in \partial I \backslash \partial_{0} I} \vec{x}(p) \cdot \vec{e}_{1} \\
= & \pi \alpha \int_{I} \vec{x} \cdot \vec{e}_{1}\left[\varkappa_{\mathcal{S}}-\bar{\varkappa}\right]^{2}\left|\vec{x}_{\rho}\right| \mathrm{d} \rho+2 \pi \lambda \int_{I}\left(\vec{x} \cdot \vec{e}_{1}\right)\left|\vec{x}_{\rho}\right| \mathrm{d} \rho+\frac{\beta}{2} \mathcal{A}_{\mathcal{S}}^{2}(t) \\
& -2 \pi \alpha_{G} \sum_{p \in \partial I \backslash \partial_{0} I} \vec{\mu}(p) \cdot \vec{e}_{1}+2 \pi \varsigma \sum_{p \in \partial I \backslash \partial_{0} I} \vec{x}(p) \cdot \vec{e}_{1},
\end{aligned}
$$

where

$$
\mathcal{A}_{\mathcal{S}}(t)=2 \pi \int_{I} \vec{x} \cdot \vec{e}_{1} \varkappa_{\mathcal{S}}\left|\vec{x}_{\rho}\right| \mathrm{d} \rho-M_{0}
$$

In this general situation, (2.14) is replaced by

$$
\mathcal{V}_{\mathcal{S}}=-\alpha \Delta_{\mathcal{S}} k_{m}+2\left[\alpha\left(k_{m}-\bar{\varkappa}\right)+\beta \mathcal{A}_{\mathcal{S}}\right] k_{g}-\left[\frac{1}{2} \alpha\left(k_{m}^{2}-\bar{\varkappa}^{2}\right)-\lambda\right] k_{m} \quad \text { on } \mathcal{S}(t),
$$

see e.g. (1.21) of [6]. A strong formulation for the flow (2.23) on $I$, on recalling (B.3) of [7], as well as (2.5) and $(2.8)$, is given by

$$
\left(\vec{x} \cdot \vec{e}_{1}\right) \vec{x}_{t} \cdot \vec{\nu}=-\alpha\left[\vec{x} \cdot \vec{e}_{1}\left(\varkappa_{\mathcal{S}}\right)_{s}\right]_{s}+2 \vec{x} \cdot \vec{e}_{1}\left[\alpha\left(\varkappa_{\mathcal{S}}-\bar{\varkappa}\right)+\beta \mathcal{A}_{\mathcal{S}}\right] \mathcal{K}_{\mathcal{S}}-\vec{x} \cdot \vec{e}_{1}\left[\frac{1}{2} \alpha\left(\varkappa_{\mathcal{S}}^{2}-\bar{\varkappa}^{2}\right)-\lambda\right] \varkappa_{\mathcal{S}} \quad \text { in } I .
$$

Next we discuss the boundary conditions associated with (2.23) and (2.24). 


\subsection{Boundary conditions}

We recall from [6] the following boundary conditions one can consider for $\mathcal{S}(t)$ on $\partial \mathcal{S}(t)$. A connected component of the boundary can either move freely, or move along the boundary of a fixed domain $\mathcal{D}$, or it will be fixed. For the latter case two types of boundary conditions arise: clamped and Navier. Corresponding to $(2.2 \mathrm{~b})-(2.2 \mathrm{~d})$, we now partition $\partial \mathcal{S}$ into $\partial_{C} \mathcal{S} \cup \partial_{N} \mathcal{S} \cup \partial_{1} \mathcal{S} \cup \partial_{2} \mathcal{S} \cup \partial_{F} \mathcal{S}$, and we also set $\partial_{S F} \mathcal{S}=\partial_{1} \mathcal{S} \cup \partial_{2} \mathcal{S}$. In the free boundary case, the three natural boundary conditions are, for $t \in(0, T]$, given by

$$
\begin{aligned}
\alpha\left(\nabla_{\mathcal{S}} k_{m}\right) \cdot \vec{\mu}_{\partial \mathcal{S}}+\varsigma k_{\partial \mathcal{S}, n} & =0 & & \text { on } \partial_{F} \mathcal{S}(t), \\
-\frac{1}{2} \alpha\left(k_{m}-\bar{\varkappa}\right)^{2}-\beta \mathcal{A}_{\mathcal{S}} k_{m}+\varsigma k_{\partial \mathcal{S}, \mu}-\alpha_{G} k_{g} & =\lambda & & \text { on } \partial_{F} \mathcal{S}(t), \\
\alpha\left(k_{m}-\bar{\varkappa}\right)+\beta \mathcal{A}_{\mathcal{S}}+\alpha_{G} k_{\mathcal{S}, n} & =0 & & \text { on } \partial_{F} \mathcal{S}(t) .
\end{aligned}
$$

In general, the term $\alpha_{G} \mathfrak{t}_{s}$ features on the right hand side of $(2.25 \mathrm{a})$, where $\mathfrak{t}=-\left(\vec{n}_{\mathcal{S}}\right)_{s} \cdot \vec{\mu}_{\partial \mathcal{S}}$ denotes the torsion of $\partial \mathcal{S}(t)$, see (1.15) and (1.22) of [6]. However, in the axisymmetric case $\partial \mathcal{S}(t)$ is made up of circles, and so the torsion is zero. For the semifree case, when $\partial_{S F} \mathcal{S}(t) \subset \partial \mathcal{D}$ for all $t \in[0, T]$, where $\partial \mathcal{D}$ is the boundary of a fixed domain $\mathcal{D} \subset \mathbb{R}^{3}$, we let $\partial \mathcal{D}$ be given by a function $F \in C^{1}\left(\mathbb{R}^{3}\right)$ such that

$$
\partial \mathcal{D}=\left\{\vec{z} \in \mathbb{R}^{3}: F(\vec{z})=0\right\} \quad \text { and } \quad|\nabla F(\vec{z})|=1 \quad \forall \vec{z} \in \partial \mathcal{D},
$$

and we denote the normal to $\mathcal{D}$ on $\partial \mathcal{D}$ by $\vec{n}_{\mathcal{D}}=\nabla F$. For the special axisymmetric setting considered here, recall $(2.2 \mathrm{~d})$, we restrict ourselves to the two cases

$$
\vec{n}_{\mathcal{D}}=\vec{n}_{1}=\frac{\overrightarrow{\mathrm{id}}-\left(\overrightarrow{\mathrm{id}} \cdot \vec{e}_{2}\right) \vec{e}_{2}}{\left|\overrightarrow{\mathrm{id}}-\left(\overrightarrow{\mathrm{id}} \cdot \vec{e}_{2}\right) \vec{e}_{2}\right|} \quad \text { on } \quad \partial_{1} \mathcal{S}(t), \quad \vec{n}_{\mathcal{D}}=\vec{n}_{2}=\vec{e}_{2} \quad \text { on } \partial_{2} \mathcal{S}(t)
$$

The semifree boundary conditions are, for $t \in(0, T]$, then

$$
\begin{aligned}
& \partial_{S F} \mathcal{S}(t) \subset \partial \mathcal{D} \\
& -\left[-\frac{1}{2} \alpha\left(k_{m}-\bar{\varkappa}\right)^{2}-\beta \mathcal{A}_{\mathcal{S}} k_{m}+\varsigma k_{\partial \mathcal{S}, \mu}-\alpha_{G} k_{g}-\lambda\right]\left(\vec{n}_{\mathcal{S}} \cdot \vec{n}_{i}\right)+\left[\alpha\left(\nabla_{\mathcal{S}} k_{m}\right) \cdot \vec{\mu}_{\partial \mathcal{S}}+\varsigma k_{\partial \mathcal{S}, n}\right]\left(\vec{\mu}_{\partial \mathcal{S}} \cdot \vec{n}_{i}\right)=0 \\
& \alpha\left(k_{m}-\bar{\varkappa}\right)+\beta \mathcal{A}_{\mathcal{S}}+\alpha_{G} k_{\partial \mathcal{S}, n}=0 \\
& \text { on } \partial_{i} \mathcal{S}(t), i=1,2,
\end{aligned}
$$

Note that compared to (1.17) and (1.22) of [6], we have once again omitted the vanishing torsion term. Clamped boundary conditions are, for $t \in(0, T]$, given by

$$
\partial_{C} \mathcal{S}(t)=\partial_{C} \mathcal{S}(0) \quad \text { and } \quad \vec{\mu}_{\partial \mathcal{S}}(t)=\vec{\zeta}_{\mathcal{S}} \quad \text { on } \quad \partial_{C} \mathcal{S}(0)
$$

where $\vec{\zeta}_{\mathcal{S}} \in C^{0}\left(\partial_{C} \mathcal{S}(0), \mathbb{S}^{2}\right)$, with $\mathbb{S}^{2}:=\left\{\vec{z} \in \mathbb{R}^{3}:|\vec{z}|=1\right\}$, needs to be axisymmetric. Similarly, Navier boundary conditions are, for $t \in(0, T]$, given by

$$
\partial_{N} \mathcal{S}(t)=\partial_{N} \mathcal{S}(0) \quad \text { and } \quad \alpha\left(k_{m}-\bar{\varkappa}\right)+\beta \mathcal{A}_{\mathcal{S}}+\alpha_{G} k_{\partial \mathcal{S}, n}=0 \quad \text { on } \partial_{N} \mathcal{S}(0) .
$$

We now translate the above boundary conditions to the axisymmetric case. On noting (2.25), (2.20), (2.19) and (2.8), we obtain, for $t \in(0, T]$, the free boundary conditions

$$
\begin{array}{r}
(-1)^{p+1} \alpha\left(\varkappa_{\mathcal{S}}\right)_{s}-\varsigma \frac{\vec{\nu} \cdot \vec{e}_{1}}{\vec{x} \cdot \vec{e}_{1}}=0 \text { on } \partial_{F} I, \\
-\frac{1}{2} \alpha\left(\varkappa_{\mathcal{S}}-\bar{\varkappa}\right)^{2}-\beta \mathcal{A}_{\mathcal{S}} \varkappa_{\mathcal{S}}-\varsigma \frac{\vec{\mu} \cdot \vec{e}_{1}}{\vec{x} \cdot \vec{e}_{1}}-\alpha_{G} \mathcal{K}_{\mathcal{S}}=\lambda \text { on } \partial_{F} I,
\end{array}
$$




$$
\alpha\left(\varkappa_{\mathcal{S}}-\bar{\varkappa}\right)+\beta \mathcal{A}_{\mathcal{S}}-\alpha_{G} \frac{\vec{\nu} \cdot \vec{e}_{1}}{\vec{x} \cdot \vec{e}_{1}}=0 \quad \text { on } \partial_{F} I
$$

Similarly, (2.27), (2.26), (2.20), (2.19) and (2.8) yield, for $t \in(0, T]$, the semifree boundary conditions

$$
\begin{aligned}
& \vec{x}(\cdot, t) \cdot \vec{e}_{i}=\vec{x}(\cdot, 0) \cdot \vec{e}_{i} \quad \text { on } \partial_{i} I, i=1,2, \\
& -\left[-\frac{1}{2} \alpha\left(\varkappa_{\mathcal{S}}-\bar{\varkappa}\right)^{2}-\beta \mathcal{A}_{\mathcal{S}} \varkappa_{\mathcal{S}}-\varsigma \frac{\vec{\mu} \cdot \vec{e}_{1}}{\vec{x} \cdot \vec{e}_{1}}-\alpha_{G} \mathcal{K}_{\mathcal{S}}-\lambda\right]\left(\vec{\nu} \cdot \vec{e}_{i}\right)+\left[(-1)^{p+1} \alpha\left(\varkappa_{\mathcal{S}}\right)_{s}-\varsigma \frac{\vec{\nu} \cdot \vec{e}_{1}}{\vec{x} \cdot \vec{e}_{1}}\right]\left(\vec{\mu} \cdot \vec{e}_{i}\right)=0 \\
& \alpha\left(\varkappa_{\mathcal{S}}-\bar{\varkappa}\right)+\beta \mathcal{A}_{\mathcal{S}}-\alpha_{G} \frac{\vec{\nu} \cdot \vec{e}_{1}}{\vec{x} \cdot \vec{e}_{1}}=0 \quad \text { on } \partial_{i} I, i=1,2
\end{aligned}
$$

Taking into account the clamped boundary conditions (2.28), we define, similarly to (2.4),

$$
\vec{\zeta}_{\mathcal{S}}\left(\vec{\Pi}_{3}^{3}(\vec{x}(p, 0), \theta)\right)=\left(\begin{array}{c}
\vec{\zeta}(p) \cdot \vec{e}_{1} \cos \theta \\
\vec{\zeta}(p) \cdot \vec{e}_{2} \\
\vec{\zeta}(p) \cdot \vec{e}_{1} \sin \theta
\end{array}\right) \quad \text { for } \quad p \in \partial_{C} I, \quad \theta \in[0,2 \pi),
$$

to be the conormal of $\mathcal{S}(t)$ on $\partial_{C} \mathcal{S}(0)=\partial_{C} \mathcal{S}(t)$. Here $\vec{\zeta}(p)$, for $p \in \partial_{C} I$, are given unit vectors that prescribe the clamping direction for the conormals $\vec{\mu}(p, t)$ of $\Gamma(t)$ at the endpoints $\vec{x}(p, t)=\vec{x}(p, 0)$, for $p \in \partial_{C} I$. In particular, for clamped boundary conditions we will enforce, for $t \in(0, T]$, on recalling $(2.20)$,

$$
\begin{aligned}
\vec{x}(p, t) & =\vec{x}(p, 0) & \text { for } & p \in \partial_{C} I, \\
(-1)^{p+1} \vec{\tau}(p, t)=\vec{\mu}(p, t) & =\vec{\zeta}(p) & \text { for } & p \in \partial_{C} I .
\end{aligned}
$$

Similarly, for Navier boundary conditions we will enforce, for $t \in(0, T]$, on recalling (2.29), (2.8) and (2.19),

$$
\begin{aligned}
\vec{x}(p, t) & =\vec{x}(p, 0) & & \text { for } \quad p \in \partial_{N} I, \\
\alpha\left(\varkappa_{\mathcal{S}}-\bar{\varkappa}\right)+\beta \mathcal{A}_{\mathcal{S}}-\alpha_{G} \frac{\vec{\nu} \cdot \vec{e}_{1}}{\vec{x} \cdot \vec{e}_{1}} & =0 & & \text { on } \partial_{N} I .
\end{aligned}
$$

Finally, we impose the following boundary conditions on $\partial_{0} I$

$$
\begin{array}{rll}
\vec{x} \cdot \vec{e}_{1}=0 & \text { on } \partial_{0} I, & \text { for } t \in[0, T], \\
\vec{x}_{\rho} \cdot \vec{e}_{2}=0 & \text { on } \partial_{0} I, & \text { for } t \in[0, T], \\
\left(\varkappa_{\mathcal{S}}\right)_{\rho}=0 & \text { on } \partial_{0} I, & \text { for } t \in(0, T] .
\end{array}
$$

Here $(2.35 \mathrm{c})$ ensures that the radially symmetric function $k_{m}$ on $\mathcal{S}(t)$ induced by $\varkappa_{\mathcal{S}}$, recall (2.8), is differentiable, while $(2.35 \mathrm{~b})$ is the same as $(2.11)$. It is natural to ask for differentiability of $k_{m}$ due to the regularisation property of parabolic equations. We also remark that $\Delta_{\mathcal{S}} k_{m}$ in (1.2), for radially symmetric solutions, is only defined even in the weak sense if $(2.35 \mathrm{c})$ holds. We note that the boundary conditions $(2.2 \mathrm{~b})-(2.2 \mathrm{~d})$ are incorporated in $(2.35 \mathrm{a}),(2.33 \mathrm{a}),(2.34 \mathrm{a})$ and $(2.31 \mathrm{a})$, respectively.

We now introduce an energy equivalent to (2.21), which takes into account the clamped, (2.33), Navier, (2.34a), and semifree, (2.31a), boundary conditions,

$$
\begin{aligned}
\widehat{E}(t)= & \widetilde{E}(t)+2 \pi \alpha_{G} \sum_{p \in \partial_{C} I} \vec{\zeta}(p) \cdot \vec{e}_{1}-2 \pi \varsigma \sum_{p \in \partial_{C} I \cup \partial_{N} I \cup \partial_{1} I} \vec{x}(p) \cdot \vec{e}_{1} \\
= & \pi \alpha \int_{I} \vec{x} \cdot \vec{e}_{1}\left[\varkappa_{\mathcal{S}}-\bar{\varkappa}^{2}\left|\vec{x}_{\rho}\right| \mathrm{d} \rho+2 \pi \lambda \int_{I}\left(\vec{x} \cdot \vec{e}_{1}\right)\left|\vec{x}_{\rho}\right| \mathrm{d} \rho+\frac{\beta}{2} \mathcal{A}_{\mathcal{S}}^{2}(t)\right. \\
& -2 \pi \alpha_{G} \sum_{p \in \partial_{M} I} \vec{\mu}(p) \cdot \vec{e}_{1}+2 \pi \varsigma \sum_{p \in \partial_{2} I \cup \partial_{F} I} \vec{x}(p) \cdot \vec{e}_{1},
\end{aligned}
$$

where $\mathcal{A}_{\mathcal{S}}$ is defined in $(2.22)$ and

$$
\partial_{M} I=\partial_{N} I \cup \partial_{S F} I \cup \partial_{F} I
$$




\subsection{Conserved flows}

In a number applications, such as biomembranes, the $L^{2}$-gradient flow of (2.15) is considered under conservation of the total surface area and, in the case of a closed surface, conservation of the enclosed volume. Before we state these variants, we recall the following useful results. We have, similarly to (2.21), that

$$
\frac{\mathrm{d}}{\mathrm{d} t} \mathcal{H}^{2}(\mathcal{S}(t))=\frac{\mathrm{d}}{\mathrm{d} t} 2 \pi \int_{I} \vec{x} \cdot \vec{e}_{1}\left|\vec{x}_{\rho}\right| \mathrm{d} \rho=2 \pi \int_{I}\left[\vec{x}_{t} \cdot \vec{e}_{1}\left|\vec{x}_{\rho}\right|+\left(\vec{x} \cdot \vec{e}_{1}\right)\left(\vec{x}_{t}\right)_{\rho} \cdot \vec{\tau}\right] \mathrm{d} \rho .
$$

In the case of a closed surface $\mathcal{S}(t)$, we have from (2.5) that

$$
\frac{\mathrm{d}}{\mathrm{d} t} \mathcal{L}^{3}(\Omega(t))=\int_{\mathcal{S}(t)} \mathcal{V}_{\mathcal{S}} \mathrm{d} \mathcal{H}^{2}=2 \pi \int_{I}\left(\vec{x} \cdot \vec{e}_{1}\right) \vec{x}_{t} \cdot \vec{\nu}\left|\vec{x}_{\rho}\right| \mathrm{d} \rho,
$$

where $\mathcal{L}^{3}$ denotes the Lebesgue measure in $\mathbb{R}^{3}, \mathcal{S}(t)=\partial \Omega(t)$, and where we assume from now on that $\vec{n}_{\mathcal{S}}$ is the outer or inner normal to $\Omega(t)$ on $\mathcal{S}(t)$, recall (2.4) and (2.3).

Generalized Helfrich flow is the surface area and volume conserving variant of (2.23), and its strong form can be stated as

$$
\mathcal{V}_{\mathcal{S}}=-\alpha \Delta_{\mathcal{S}} k_{m}+2\left[\alpha\left(k_{m}-\bar{\varkappa}\right)+\beta \mathcal{A}_{\mathcal{S}}\right] k_{g}-\left[\frac{1}{2} \alpha\left(k_{m}^{2}-\bar{\varkappa}^{2}\right)-\lambda\right] k_{m}+\lambda_{A} k_{m}-\lambda_{V} \quad \text { on } \mathcal{S}(t),
$$

where $\left(\lambda_{A}(t), \lambda_{V}(t)\right)^{T} \in \mathbb{R}^{2}$ are chosen such that

$$
\frac{\mathrm{d}}{\mathrm{d} t} \mathcal{H}^{2}(\mathcal{S}(t))=0, \quad \frac{\mathrm{d}}{\mathrm{d} t} \mathcal{L}^{3}(\Omega(t))=0
$$

For axisymmetric surfaces the flow (2.40) with (2.41) can be equivalently formulated as

$$
\begin{aligned}
\left(\vec{x} \cdot \vec{e}_{1}\right) \vec{x}_{t} \cdot \vec{\nu}= & -\alpha\left[\vec{x} \cdot \vec{e}_{1}\left(\varkappa_{\mathcal{S}}\right)_{\mathcal{S}}\right]_{S}+2 \vec{x} \cdot \vec{e}_{1}\left[\alpha\left(\varkappa_{\mathcal{S}}-\bar{\varkappa}\right)+\beta \mathcal{A}_{\mathcal{S}}\right] \mathcal{K}_{\mathcal{S}}-\vec{x} \cdot \vec{e}_{1}\left[\frac{1}{2} \alpha\left(\varkappa_{\mathcal{S}}^{2}-\bar{\varkappa}^{2}\right)-\lambda\right] \varkappa_{\mathcal{S}} \\
& +\lambda_{A} \vec{x} \cdot \vec{e}_{1} \varkappa_{\mathcal{S}}-\lambda_{V} \vec{x} \cdot \vec{e}_{1} \text { in } I,
\end{aligned}
$$

where $\left(\lambda_{A}(t), \lambda_{V}(t)\right)^{T} \in \mathbb{R}^{2}$ are chosen such that

$$
\int_{I}\left[\vec{x}_{t} \cdot \vec{e}_{1}\left|\vec{x}_{\rho}\right|+\left(\vec{x} \cdot \vec{e}_{1}\right)\left(\vec{x}_{t}\right)_{\rho} \cdot \vec{\tau}\right] \mathrm{d} \rho=0, \quad \int_{I}\left(\vec{x} \cdot \vec{e}_{1}\right) \vec{x}_{t} \cdot \vec{\nu}\left|\vec{x}_{\rho}\right| \mathrm{d} \rho=0,
$$

where we recall (2.24), (2.38) and (2.39).

\section{WEAK FORMULATIONS}

On recalling (2.3), we have for all $\vec{a}, \vec{b} \in \mathbb{R}^{2}$ that

$$
\begin{aligned}
\vec{a} \cdot \vec{b}^{\perp} & =-\vec{a}^{\perp} \cdot \vec{b}, \\
\vec{a}^{\perp} & =\left(\vec{a}^{\perp} \cdot \vec{\tau}\right) \vec{\tau}+\left(\vec{a}^{\perp} \cdot \vec{\nu}\right) \vec{\nu}=\left(\vec{a}^{\perp} \cdot \vec{\nu}^{\perp}\right) \vec{\tau}-\left(\vec{a}^{\perp} \cdot \vec{\tau}^{\perp}\right) \vec{\nu}=(\vec{a} \cdot \vec{\nu}) \vec{\tau}-(\vec{a} \cdot \vec{\tau}) \vec{\nu} .
\end{aligned}
$$

We define the first variation of a differentiable quantity $B(\vec{x})$, in the direction $\vec{\chi}$ as

$$
\left[\frac{\delta}{\delta \vec{x}} B(\vec{x})\right](\vec{\chi})=\lim _{\varepsilon \rightarrow 0} \frac{B(\vec{x}+\varepsilon \vec{\chi})-B(\vec{x})}{\varepsilon} .
$$

For later use, on noting (3.2) and (2.3), we observe that

$$
\left[\frac{\delta}{\delta \vec{x}}\left|\vec{x}_{\rho}\right|\right](\vec{\chi})=\frac{\vec{x}_{\rho} \cdot \vec{\chi}_{\rho}}{\left|\vec{x}_{\rho}\right|}=\vec{\tau} \cdot \vec{\chi}_{\rho}=\vec{\tau} \cdot \vec{\chi}_{s}\left|\vec{x}_{\rho}\right|,
$$




$$
\begin{aligned}
{\left[\frac{\delta}{\delta \vec{x}} \vec{\tau}\right](\vec{\chi}) } & =\left[\frac{\delta}{\delta \vec{x}} \frac{\vec{x}_{\rho}}{\left|\vec{x}_{\rho}\right|}\right](\vec{\chi})=\frac{\vec{\chi}_{\rho}}{\left|\vec{x}_{\rho}\right|}-\frac{\vec{x}_{\rho}}{\left|\vec{x}_{\rho}\right|^{2}} \frac{\vec{x}_{\rho} \cdot \vec{\chi}_{\rho}}{\left|\vec{x}_{\rho}\right|}=\vec{\chi}_{s}-\vec{\tau}\left(\vec{\chi}_{s} \cdot \vec{\tau}\right)=\left(\vec{\chi}_{s} \cdot \vec{\nu}\right) \vec{\nu}, \\
{\left[\frac{\delta}{\delta \vec{x}} \vec{\nu}\right](\vec{\chi}) } & =-\left[\frac{\delta}{\delta \vec{x}} \vec{\tau}^{\perp}\right](\vec{\chi})=-\left(\vec{\chi}_{s} \cdot \vec{\nu}\right) \vec{\nu}^{\perp}=-\left(\vec{\chi}_{s} \cdot \vec{\nu}\right) \vec{\tau}, \\
{\left[\frac{\delta}{\delta \vec{x}} \vec{\nu}\left|\vec{x}_{\rho}\right|\right](\vec{\chi}) } & =-\left[\frac{\delta}{\delta \vec{x}} \vec{x}_{\rho}^{\perp}\right](\vec{\chi})=-\vec{\chi}_{\rho}^{\perp}=-\vec{\chi}_{s}^{\perp}\left|\vec{x}_{\rho}\right|,
\end{aligned}
$$

where we always assume that $\vec{\chi}$ is sufficiently smooth so that all the quantities are defined almost everywhere; e.g. $\vec{\chi} \in\left[W^{1, \infty}(I)\right]^{2}$. In addition, we note that

$$
\left[\frac{\delta}{\delta \vec{x}} B(\vec{x})\right]\left(\vec{x}_{t}\right)=\frac{\mathrm{d}}{\mathrm{d} t} B(\vec{x})
$$

Let

$$
\begin{aligned}
\underline{V}_{\partial_{0}} & =\left\{\vec{\eta} \in\left[H^{1}(I)\right]^{2}: \vec{\eta}(\rho) \cdot \vec{e}_{1}=0 \quad \forall \rho \in \partial_{0} I\right\}, \\
\mathbb{X} & =\left\{\vec{\eta} \in \underline{V}_{\partial_{0}}: \vec{\eta}(\rho)=\overrightarrow{0} \quad \forall \rho \in \partial_{C} I \cup \partial_{N} I, \vec{\eta}(\rho) \cdot \vec{e}_{i}=0 \quad \forall \rho \in \partial_{i} I, i=1,2\right\} .
\end{aligned}
$$

Here $\mathbb{X}$ is the space of all directions in which we can vary a given curve. For a given $\vec{z} \in \mathbb{R}^{2}$, on recalling (2.37), we define

$$
\mathbb{Y}(\vec{z})=\left\{\vec{\eta} \in \underline{V}_{\partial_{0}}: \vec{\eta}(\rho)=\vec{z} \quad \forall \rho \in \partial_{M} I\right\} .
$$

On recalling (2.37), we note that

$$
\text { if } \partial_{C} I=\emptyset \text { then } \mathbb{Y}(\overrightarrow{0}) \subset \mathbb{X} .
$$

Let $(\cdot, \cdot)$ denote the $L^{2}$-inner product on $I$. We now consider the following weak formulation of (2.6) with $\vec{x} \in \underline{V}_{\partial_{0}}$ and $\varkappa \in L^{2}(I)$ such that

$$
\left(\varkappa \vec{\nu}, \vec{\eta}\left|\vec{x}_{\rho}\right|\right)+\left(\vec{\tau}, \vec{\eta}_{\rho}\right)=\sum_{p \in \partial_{C} I}[\vec{\zeta} \cdot \vec{\eta}](p)+\sum_{p \in \partial_{M} I}[\vec{m} \cdot \vec{\eta}](p) \quad \forall \vec{\eta} \in \underline{V}_{\partial_{0}},
$$

where we recall (2.3). We note that (3.8) weakly imposes $(2.35 \mathrm{~b})$ and $(2.33 \mathrm{~b})$, where $\vec{\zeta}(p) \in \mathbb{S}^{1}, p \in \partial_{C} I$, are given data. However, (3.8) also yields that $\vec{m}(p)=\vec{\mu}(p) \in \mathbb{R}^{2}, p \in \partial_{M} I$. This will not be the case under discretization, where $\vec{m}(p) \in \mathbb{R}^{2}, p \in \partial_{M} I$, are approximations to the conormals $\vec{\mu}(p), p \in \partial_{M} I$.

Similarly, we consider the following weak formulation of (2.9) with $\vec{x} \in \underline{V}_{\partial_{0}}$ and $\varkappa_{\mathcal{S}} \in L^{2}(I)$ such that

$$
\left(\vec{x} \cdot \vec{e}_{1} \varkappa_{\mathcal{S}} \vec{\nu}+\vec{e}_{1}, \vec{\eta}\left|\vec{x}_{\rho}\right|\right)+\left(\left(\vec{x} \cdot \vec{e}_{1}\right) \vec{\tau}, \vec{\eta}_{\rho}\right)=\sum_{p \in \partial_{C} I}\left[\left(\vec{x} \cdot \vec{e}_{1}\right) \vec{\zeta} \cdot \vec{\eta}\right](p)+\sum_{p \in \partial_{M} I}\left[\left(\vec{x} \cdot \vec{e}_{1}\right) \vec{m} \cdot \vec{\eta}\right](p) \quad \forall \vec{\eta} \in \underline{V}_{\partial_{0}}
$$

It is shown in Appendix A of [9] that, despite the degenerate weight, (3.9) weakly imposes (2.11). In addition, (3.9) weakly imposes $(2.33 \mathrm{~b})$.

\subsection{Based on $\varkappa$}

We begin with a weak formulation based on (3.8). Finite element approximations based on this weak formulation will exhibit an equidistribution property.

On recalling (2.36), (2.22), (2.7), (3.8) and that $\vec{\mu}=\vec{m}$ on $\partial_{M} I$, we define the Lagrangian

$$
\mathcal{L}\left(\vec{x}, \varkappa^{\star}, \vec{m}, \vec{y}\right)=\pi\left(\alpha\left[\varkappa^{\star}-\frac{\vec{\nu} \cdot \vec{e}_{1}}{\vec{x} \cdot \vec{e}_{1}}-\bar{\varkappa}\right]^{2}+2 \lambda, \vec{x} \cdot \vec{e}_{1}\left|\vec{x}_{\rho}\right|\right)+\frac{\beta}{2}\left[2 \pi\left(\varkappa^{\star}-\frac{\vec{\nu} \cdot \vec{e}_{1}}{\vec{x} \cdot \vec{e}_{1}}, \vec{x} \cdot \vec{e}_{1}\left|\vec{x}_{\rho}\right|\right)-M_{0}\right]^{2}
$$




$$
\begin{aligned}
& -\left(\varkappa^{\star} \vec{\nu}, \vec{y}\left|\vec{x}_{\rho}\right|\right)-\left(\vec{\tau}, \vec{y}_{\rho}\right)+2 \pi \varsigma \sum_{p \in \partial_{2} I \cup \partial_{F} I} \vec{x}(p) \cdot \vec{e}_{1}+\sum_{p \in \partial_{C} I}[\vec{\zeta} \cdot \vec{y}](p) \\
& +\sum_{p \in \partial_{M} I}\left[\vec{m} \cdot\left(\vec{y}-2 \pi \alpha_{G} \vec{e}_{1}\right)\right](p),
\end{aligned}
$$

for $\vec{x} \in \underline{V}_{\partial_{0}}, \varkappa^{\star} \in L^{2}(I), \vec{m}: \partial_{M} I \rightarrow \mathbb{R}^{2}$ and $\vec{y} \in \underline{V}_{\partial_{0}}$. Here, we recall from (2.2b) and (2.12) that on the continuous level the Lagrangian (3.10) is well-defined for curves generating a smooth surface also in the case $\partial_{0} I \neq \emptyset$.

Taking variations $\vec{\eta} \in \underline{V}_{\partial_{0}}$ in $\vec{y}$, and setting $\left[\frac{\delta}{\delta \vec{y}} \mathcal{L}\right](\vec{\eta})=0$ we obtain

$$
\left(\varkappa^{\star} \vec{\nu}, \vec{\eta}\left|\vec{x}_{\rho}\right|\right)+\left(\vec{\tau}, \vec{\eta}_{\rho}\right)=\sum_{p \in \partial_{C} I}[\vec{\zeta} \cdot \vec{\eta}](p)+\sum_{p \in \partial_{M} I}[\vec{m} \cdot \vec{\eta}](p) \quad \forall \vec{\eta} \in \underline{V}_{\partial_{0}},
$$

and so combining with (3.8) yields that $\varkappa^{\star}=\varkappa$. We are going to use this identity from now on. Taking variations $\chi \in L^{2}(I)$ in $\varkappa^{\star}$ and setting $\left[\frac{\delta}{\delta \varkappa^{\star}} \mathcal{L}\right](\chi)=0$ we obtain, on using $\varkappa^{\star}=\varkappa$, that

$$
2 \pi\left(\alpha\left[\varkappa-\frac{\vec{\nu} \cdot \vec{e}_{1}}{\vec{x} \cdot \vec{e}_{1}}-\bar{\varkappa}\right]+\beta \mathcal{A}, \vec{x} \cdot \vec{e}_{1} \chi\left|\vec{x}_{\rho}\right|\right)-\left(\vec{\nu} \cdot \vec{y}, \chi\left|\vec{x}_{\rho}\right|\right)=0 \quad \forall \chi \in L^{2}(I),
$$

where

$$
\mathcal{A}(t)=2 \pi\left(\varkappa-\frac{\vec{\nu} \cdot \vec{e}_{1}}{\vec{x} \cdot \vec{e}_{1}}, \vec{x} \cdot \vec{e}_{1}\left|\vec{x}_{\rho}\right|\right)-M_{0}=2 \pi\left(\vec{x} \cdot \vec{e}_{1} \varkappa-\vec{\nu} \cdot \vec{e}_{1},\left|\vec{x}_{\rho}\right|\right)-M_{0}
$$

We note that

$$
\left[\frac{\delta}{\delta \vec{x}} \mathcal{A}(t)\right](\vec{\chi})=2 \pi\left(\varkappa,\left[\frac{\delta}{\delta \vec{x}} \vec{x} \cdot \vec{e}_{1}\left|\vec{x}_{\rho}\right|\right](\vec{\chi})\right)-2 \pi\left(\vec{e}_{1},\left[\frac{\delta}{\delta \vec{x}} \vec{\nu}\left|\vec{x}_{\rho}\right|\right](\vec{\chi})\right) \quad \forall \vec{\chi} \in \mathbb{X} .
$$

Taking variations in $\vec{m}$, and setting them to zero, yields that

$$
\vec{y}=2 \pi \alpha_{G} \vec{e}_{1} \quad \text { on } \partial_{M} I .
$$

Taking variations $\vec{\chi} \in \mathbb{X}$ in $\vec{x}$, and setting $2 \pi\left(\left(\vec{x} \cdot \vec{e}_{1}\right) \vec{x}_{t} \cdot \vec{\nu}, \vec{\chi} \cdot \vec{\nu}\left|\vec{x}_{\rho}\right|\right)=-\left[\frac{\delta}{\delta \vec{x}} \mathcal{L}\right](\vec{\chi})$ we obtain, on noting (3.14) and $(3.5 \mathrm{~b})$, that

$$
\begin{aligned}
2 \pi\left(\left(\vec{x} \cdot \vec{e}_{1}\right) \vec{x}_{t} \cdot \vec{\nu}, \vec{\chi} \cdot \vec{\nu}\left|\vec{x}_{\rho}\right|\right)= & -\pi\left(\alpha\left[\varkappa-\frac{\vec{\nu} \cdot \vec{e}_{1}}{\vec{x} \cdot \vec{e}_{1}}-\bar{\varkappa}\right]^{2}+2 \lambda+2 \beta \mathcal{A} \varkappa,\left[\frac{\delta}{\delta \vec{x}}\left(\vec{x} \cdot \vec{e}_{1}\right)\left|\vec{x}_{\rho}\right|\right](\vec{\chi})\right) \\
& +2 \pi \alpha\left(\varkappa-\frac{\vec{\nu} \cdot \vec{e}_{1}}{\vec{x} \cdot \vec{e}_{1}}-\bar{\varkappa}, \vec{x} \cdot \vec{e}_{1}\left[\frac{\delta}{\delta \vec{x}} \frac{\vec{\nu} \cdot \vec{e}_{1}}{\vec{x} \cdot \vec{e}_{1}}\right](\vec{\chi})\left|\vec{x}_{\rho}\right|\right) \\
& +\left(\varkappa \vec{y}+2 \pi \beta \mathcal{A} \vec{e}_{1},\left[\frac{\delta}{\delta \vec{x}} \vec{\nu}\left|\vec{x}_{\rho}\right|\right](\vec{\chi})\right)+\left(\vec{y}_{\rho},\left[\frac{\delta}{\delta \vec{x}} \vec{\tau}\right](\vec{\chi})\right) \\
& -2 \pi \varsigma \sum_{p \in \partial_{2} I \cup \partial_{F} I} \vec{\chi}(p) \cdot \vec{e}_{1} \quad \forall \vec{\chi} \in \mathbb{X} .
\end{aligned}
$$

Choosing $\vec{\chi}=\vec{x}_{t} \in \mathbb{X}$ in (3.16) yields, on noting (3.4), that

$$
\begin{aligned}
2 \pi\left(\vec{x} \cdot \vec{e}_{1}\left(\vec{x}_{t} \cdot \vec{\nu}\right)^{2},\left|\vec{x}_{\rho}\right|\right)= & -\pi\left(\alpha\left[\varkappa-\frac{\vec{\nu} \cdot \vec{e}_{1}}{\vec{x} \cdot \vec{e}_{1}}-\bar{\varkappa}\right]^{2}+2 \lambda+2 \beta \mathcal{A} \varkappa,\left[\left(\vec{x} \cdot \vec{e}_{1}\right)\left|\vec{x}_{\rho}\right|\right]_{t}\right) \\
& +2 \pi \alpha\left(\varkappa-\frac{\vec{\nu} \cdot \vec{e}_{1}}{\vec{x} \cdot \vec{e}_{1}}-\bar{\varkappa}, \vec{x} \cdot \vec{e}_{1}\left[\frac{\vec{\nu} \cdot \vec{e}_{1}}{\vec{x} \cdot \vec{e}_{1}}\right]_{t}\left|\vec{x}_{\rho}\right|\right)+\left(\varkappa \vec{y}+2 \pi \beta \mathcal{A} \vec{e}_{1},\left[\vec{\nu}\left|\vec{x}_{\rho}\right|\right]_{t}\right)
\end{aligned}
$$




$$
+\left(\vec{y}_{\rho}, \vec{\tau}_{t}\right)-2 \pi \varsigma \sum_{p \in \partial_{2} I \cup \partial_{F} I} \vec{x}_{t}(p) \cdot \vec{e}_{1}
$$

Differentiating (3.11) with respect to time, and then choosing $\vec{\eta}=\vec{y}$, on recalling $\varkappa^{\star}=\varkappa$ and $\vec{\zeta}$ is independent of $t$, yields that

$$
\left(\varkappa_{t}, \vec{y} \cdot \vec{\nu}\left|\vec{x}_{\rho}\right|\right)+\left(\varkappa \vec{y},\left[\vec{\nu}\left|\vec{x}_{\rho}\right|\right]_{t}\right)+\left(\vec{\tau}_{t}, \vec{y}_{\rho}\right)=\sum_{p \in \partial_{M} I}\left[\vec{m}_{t} \cdot \vec{y}\right](p) .
$$

It follows from (3.18), (3.15) and (3.12) with $\chi=\varkappa_{t} \in L^{2}(I)$ that

$$
\begin{aligned}
\left(\varkappa \vec{y},\left[\vec{\nu}\left|\vec{x}_{\rho}\right|\right]_{t}\right)+\left(\vec{\tau}_{t}, \vec{y}_{\rho}\right) & =-\left(\varkappa t, \vec{y} \cdot \vec{\nu}\left|\vec{x}_{\rho}\right|\right)+2 \pi \alpha_{G} \sum_{p \in \partial_{M} I} \vec{m}_{t}(p) \cdot \vec{e}_{1} \\
& =-2 \pi\left(\alpha\left[\varkappa-\frac{\vec{\nu} \cdot \vec{e}_{1}}{\vec{x} \cdot \vec{e}_{1}}-\bar{\varkappa}\right]+\beta \mathcal{A}, \vec{x} \cdot \vec{e}_{1} \varkappa_{t}\left|\vec{x}_{\rho}\right|\right)+2 \pi \alpha_{G} \sum_{p \in \partial_{M} I} \vec{m}_{t}(p) \cdot \vec{e}_{1} .
\end{aligned}
$$

Combining (3.17) and (3.19) yields that

$$
\begin{aligned}
2 \pi\left(\vec{x} \cdot \vec{e}_{1}\left(\vec{x}_{t} \cdot \vec{\nu}\right)^{2},\left|\vec{x}_{\rho}\right|\right)= & -\pi\left(\alpha\left[\varkappa-\frac{\vec{\nu} \cdot \vec{e}_{1}}{\vec{x} \cdot \vec{e}_{1}}-\bar{\varkappa}\right]^{2}+2 \lambda+2 \beta \mathcal{A} \varkappa,\left[\left(\vec{x} \cdot \vec{e}_{1}\right)\left|\vec{x}_{\rho}\right|\right]_{t}\right) \\
& +2 \pi \alpha\left(\varkappa-\frac{\vec{\nu} \cdot \vec{e}_{1}}{\vec{x} \cdot \vec{e}_{1}}-\bar{\varkappa}, \vec{x} \cdot \vec{e}_{1}\left[\frac{\vec{\nu} \cdot \vec{e}_{1}}{\vec{x} \cdot \vec{e}_{1}}\right]_{t}\left|\vec{x}_{\rho}\right|\right)+2 \pi \beta \mathcal{A}\left(\vec{e}_{1},\left[\vec{\nu}\left|\vec{x}_{\rho}\right|\right]_{t}\right) \\
& -2 \pi\left(\alpha\left[\varkappa-\frac{\vec{\nu} \cdot \vec{e}_{1}}{\vec{x} \cdot \vec{e}_{1}}-\bar{\varkappa}\right]+\beta \mathcal{A}, \vec{x} \cdot \vec{e}_{1} \varkappa_{t}\left|\vec{x}_{\rho}\right|\right)+2 \pi \alpha_{G} \sum_{p \in \partial_{M} I} \vec{m}_{t}(p) \cdot \vec{e}_{1} \\
& -2 \pi \varsigma \sum_{p \in \partial_{2} I \cup \partial_{F} I} \vec{x}_{t}(p) \cdot \vec{e}_{1} \\
= & -\pi \frac{\mathrm{d}}{\mathrm{d} t}\left[\alpha\left[\varkappa-\frac{\vec{\nu} \cdot \vec{e}_{1}}{\vec{x} \cdot \vec{e}_{1}}-\bar{\varkappa}\right]^{2}+2 \lambda, \vec{x} \cdot \vec{e}_{1}\left|\vec{x}_{\rho}\right|\right]-\frac{\beta}{2} \frac{\mathrm{d}}{\mathrm{d} t} \mathcal{A}^{2}-2 \pi \varsigma \sum_{p \in \partial_{2} I \cup \partial_{F} I} \vec{x}_{t}(p) \cdot \vec{e}_{1} \\
& +2 \pi \alpha_{G} \sum_{p \in \partial_{M} I} \vec{m}_{t}(p) \cdot \vec{e}_{1} \\
= & -\frac{\mathrm{d}}{\mathrm{d} t} \widehat{E}(t)
\end{aligned}
$$

where we have recalled (2.36), (2.22), (2.7), (3.13) and that $\vec{\mu}=\vec{m}$ on $\partial_{M} I$.

Remark 3.1. The property $\frac{\mathrm{d}}{\mathrm{d} t} \widehat{E}(t)+2 \pi\left(\vec{x} \cdot \vec{e}_{1}\left(\vec{x}_{t} \cdot \vec{\nu}\right)^{2},\left|\vec{x}_{\rho}\right|\right)=0$ shown in (3.20) demonstrates the gradient flow property of the derived weak formulation.

We now return to (3.16), which, on recalling (3.3), can be rewritten as

$$
\begin{aligned}
2 \pi\left(\left(\vec{x} \cdot \vec{e}_{1}\right) \vec{x}_{t} \cdot \vec{\nu}, \vec{\chi} \cdot \vec{\nu}\left|\vec{x}_{\rho}\right|\right)= & -\pi\left(\alpha\left[\varkappa-\frac{\vec{\nu} \cdot \vec{e}_{1}}{\vec{x} \cdot \vec{e}_{1}}-\bar{\varkappa}\right]^{2}+2 \lambda+2 \beta \mathcal{A} \varkappa, \vec{\chi} \cdot \vec{e}_{1}\left|\vec{x}_{\rho}\right|+\left(\vec{x} \cdot \vec{e}_{1}\right) \vec{\tau} \cdot \vec{\chi}_{\rho}\right) \\
& -2 \pi \alpha\left(\varkappa-\frac{\vec{\nu} \cdot \vec{e}_{1}}{\vec{x} \cdot \vec{e}_{1}}-\bar{\varkappa}, \frac{\vec{\nu} \cdot \vec{e}_{1}}{\vec{x} \cdot \vec{e}_{1}} \vec{\chi} \cdot \vec{e}_{1}\left|\vec{x}_{\rho}\right|\right)-2 \pi \alpha\left(\varkappa-\frac{\vec{\nu} \cdot \vec{e}_{1}}{\vec{x} \cdot \vec{e}_{1}}-\bar{\varkappa},\left(\vec{\chi}_{\rho} \cdot \vec{\nu}\right) \vec{\tau} \cdot \vec{e}_{1}\right) \\
& -\left(\varkappa \vec{y}+2 \pi \beta \mathcal{A} \vec{e}_{1}, \vec{\chi}_{\rho}^{\perp}\right)+\left(\vec{y}_{\rho} \cdot \vec{\nu}, \vec{\chi}_{\rho} \cdot \vec{\nu}\left|\vec{x}_{\rho}\right|^{-1}\right) \\
& -2 \pi \varsigma \sum_{p \in \partial_{2} I \cup \partial_{F} I} \vec{\chi}(p) \cdot \vec{e}_{1} \quad \forall \vec{\chi} \in \mathbb{X} .
\end{aligned}
$$


Our finite element approximation is going to be based on the following formulation, on combining (3.21), (3.12), (3.11), (3.15) and (3.13), and on recalling $\varkappa^{\star}=\varkappa,(3.1 \mathrm{a})$ and (2.3).

$(\mathcal{P})$ Let $\vec{x}(\cdot, 0) \in \underline{V}_{\partial_{0}}$ and $\alpha \in \mathbb{R}_{>0}, \bar{\varkappa}, M_{0}, \alpha_{G}, \lambda, \varsigma \in \mathbb{R}, \beta \in \mathbb{R}_{\geq 0}, \vec{\zeta}: \partial_{C} I \rightarrow \mathbb{S}^{1}$ be given. For $t \in(0, T]$, find $\vec{x}(\cdot, t) \in \underline{V}_{\partial_{0}}$, with $\overrightarrow{\vec{x}_{t}}(\cdot, t) \in \mathbb{X}, \varkappa(\cdot, t) \in L^{2}(I), \vec{y}(\cdot, t) \in \mathbb{Y}\left(2 \pi \alpha_{G} \vec{e}_{1}\right)$ and $\vec{m}(\cdot, t): \partial_{M} I \rightarrow \mathbb{R}^{2}$ such that

$$
\begin{aligned}
& 2 \pi\left(\left(\vec{x} \cdot \vec{e}_{1}\right) \vec{x}_{t} \cdot \vec{\nu}, \vec{\chi} \cdot \vec{\nu}\left|\vec{x}_{\rho}\right|\right)-\left(\vec{y}_{\rho} \cdot \vec{\nu}, \vec{\chi}_{\rho} \cdot \vec{\nu}\left|\vec{x}_{\rho}\right|^{-1}\right) \\
&=-\pi\left(\alpha\left[\varkappa-\frac{\vec{\nu} \cdot \vec{e}_{1}}{\vec{x} \cdot \vec{e}_{1}}-\bar{\varkappa}\right]^{2}+2 \lambda+2 \beta \mathcal{A} \varkappa, \vec{\chi} \cdot \vec{e}_{1}\left|\vec{x}_{\rho}\right|+\left(\vec{x} \cdot \vec{e}_{1}\right) \vec{\tau} \cdot \vec{\chi}_{\rho}\right) \\
&-2 \pi \alpha\left(\varkappa-\frac{\vec{\nu} \cdot \vec{e}_{1}}{\vec{x} \cdot \vec{e}_{1}}-\bar{\varkappa}, \frac{\vec{\nu} \cdot \vec{e}_{1}}{\vec{x} \cdot \vec{e}_{1}} \vec{\chi} \cdot \vec{e}_{1}\left|\vec{x}_{\rho}\right|\right) \\
& \quad-2 \pi \alpha\left(\varkappa-\frac{\vec{\nu} \cdot \vec{e}_{1}}{\vec{x} \cdot \vec{e}_{1}}-\bar{\varkappa},\left(\vec{\tau} \cdot \vec{e}_{1}\right) \vec{\chi}_{\rho} \cdot \vec{\nu}\right)+\left(\varkappa \vec{y}^{\perp}-2 \pi \beta \mathcal{A} \vec{e}_{2}, \vec{\chi}_{\rho}\right)-2 \pi \varsigma \sum_{p \in \partial_{2} I \cup \partial_{F} I} \vec{\chi}(p) \cdot \vec{e}_{1} \quad \forall \vec{\chi} \in \mathbb{X} \\
& 2 \pi\left(\alpha\left[\varkappa-\frac{\vec{\nu} \cdot \vec{e}_{1}}{\vec{x} \cdot \vec{e}_{1}}-\bar{\varkappa}\right]+\beta \mathcal{A}, \vec{x} \cdot \vec{e}_{1} \chi\left|\vec{x}_{\rho}\right|\right)-\left(\vec{\nu} \cdot \vec{y}, \chi\left|\vec{x}_{\rho}\right|\right)=0 \quad \forall \chi \in L^{2}(I) \\
&\left(\varkappa \vec{\nu}, \vec{\eta}\left|\vec{x}_{\rho}\right|\right)+\left(\vec{x}_{\rho}, \vec{\eta}_{\rho}\left|\vec{x}_{\rho}\right|^{-1}\right)=\sum_{p \in \partial_{C} I}[\vec{\zeta} \cdot \vec{\eta}](p)+\sum_{p \in \partial_{M} I}[\vec{m} \cdot \vec{\eta}](p) \quad \forall \vec{\eta} \in \underline{V}_{\partial_{0}}
\end{aligned}
$$

where $\mathcal{A}(t)$ is given by (3.13). We note that the number of unknowns fixed via $\vec{y} \in \mathbb{Y}\left(2 \pi \alpha_{G} \vec{e}_{1}\right)$ on $\partial_{M} I$ is matched by the new degrees of freedom arising from $\{\vec{m}(p)\}_{p \in \partial_{M} I}$.

We remark that (3.22) is independent of the tangential part $\vec{y} \cdot \vec{\tau}$ of $\vec{y}$. To see this, we note that it follows from (2.3) and (2.6) that

$$
\left(\vec{y}_{s} \cdot \vec{\nu}\right) \vec{\nu}=(\vec{y} \cdot \vec{\nu})_{s} \vec{\nu}-\left(\vec{y} \cdot \vec{\nu}_{s}\right) \vec{\nu}=(\vec{y} \cdot \vec{\nu})_{s} \vec{\nu}+\varkappa(\vec{y} \cdot \vec{\tau}) \vec{\nu} \quad \text { in } \bar{I}
$$

Hence the only terms involving $\vec{y}$ in (3.22a) are

$$
\varkappa \vec{y}^{\perp}+\left(\vec{y}_{s} \cdot \vec{\nu}\right) \vec{\nu}=\varkappa\left(\vec{y}^{\perp}+(\vec{y} \cdot \vec{\tau}) \vec{\nu}\right)+(\vec{y} \cdot \vec{\nu})_{s} \vec{\nu}=\varkappa(\vec{y} \cdot \vec{\nu}) \vec{\tau}+(\vec{y} \cdot \vec{\nu})_{s} \vec{\nu} \quad \text { in } \bar{I}
$$

where we have recalled (3.1b). This shows that (3.22) only depends on $\vec{y} \cdot \vec{\nu}$, and not on $\vec{y} \cdot \vec{\tau}$. We refer to Appendix A.1, where we show that (3.22) for a sufficiently smooth solution gives rise to the strong form (2.24) and (2.6).

\subsubsection{Conserved flows}

In this subsection we present a weak formulation for the conserving flow (2.40). To this end, we assume that the hypersurface $\mathcal{S}(t)$ has no boundary, and so

$$
\partial I=\partial_{0} I \quad \Longrightarrow \quad \mathbb{X}=\underline{V}_{\partial_{0}}
$$

Then, on writing (3.22a) as

$$
2 \pi\left(\left(\vec{x} \cdot \vec{e}_{1}\right) \vec{x}_{t} \cdot \vec{\nu}, \vec{\chi} \cdot \vec{\nu}\left|\vec{x}_{\rho}\right|\right)-\left(\vec{y}_{\rho} \cdot \vec{\nu}, \vec{\chi}_{\rho} \cdot \vec{\nu}\left|\vec{x}_{\rho}\right|^{-1}\right)=\left(\vec{f}, \vec{\chi}\left|\vec{x}_{\rho}\right|\right) \quad \forall \vec{\chi} \in \mathbb{X}
$$

a weak formulation of $(2.42)$ and (2.43) is given by (3.22), with (3.22a) replaced by

$$
\begin{aligned}
& 2 \pi\left(\left(\vec{x} \cdot \vec{e}_{1}\right) \vec{x}_{t} \cdot \vec{\nu}, \vec{\chi} \cdot \vec{\nu}\left|\vec{x}_{\rho}\right|\right)-\left(\vec{y}_{\rho} \cdot \vec{\nu}, \vec{\chi}_{\rho} \cdot \vec{\nu}\left|\vec{x}_{\rho}\right|^{-1}\right)=\left(\vec{f}, \vec{\chi}\left|\vec{x}_{\rho}\right|\right) \\
& \quad-2 \pi \lambda_{A}\left[\left(\vec{e}_{1}, \vec{\chi}\left|\vec{x}_{\rho}\right|\right)+\left(\left(\vec{x} \cdot \vec{e}_{1}\right) \vec{\tau}, \vec{\chi}_{\rho}\right)\right]-2 \pi \lambda_{V}\left(\left(\vec{x} \cdot \vec{e}_{1}\right) \vec{\nu}, \vec{\chi}\left|\vec{x}_{\rho}\right|\right) \quad \forall \vec{\chi} \in \mathbb{X},
\end{aligned}
$$


where $\left(\lambda_{A}(t), \lambda_{V}(t)\right)^{T} \in \mathbb{R}^{2}$ are chosen such that (2.43) holds.

Choosing $\vec{\eta}=\left(\vec{x} \cdot \vec{e}_{1}\right) \vec{x}_{t} \in \mathbb{X}=\underline{V}_{\partial_{0}}$ in (3.22c) and noting (2.3) yields that

$$
\begin{aligned}
\left(\vec{e}_{1}, \vec{x}_{t}\left|\vec{x}_{\rho}\right|\right)+\left(\left(\vec{x} \cdot \vec{e}_{1}\right) \vec{\tau},\left(\vec{x}_{t}\right)_{\rho}\right) & =-\left(\left(\vec{x} \cdot \vec{e}_{1}\right) \varkappa, \vec{x}_{t} \cdot \vec{\nu}\left|\vec{x}_{\rho}\right|\right)+\left(\vec{x}_{t} \cdot\left[\vec{e}_{1}-\left(\vec{e}_{1} \cdot \vec{\tau}\right) \vec{\tau}\right],\left|\vec{x}_{\rho}\right|\right) \\
& =-\left(\vec{x} \cdot \vec{e}_{1}\left(\varkappa-\frac{\vec{\nu} \cdot \vec{e}_{1}}{\vec{x} \cdot \vec{e}_{1}}\right), \vec{x}_{t} \cdot \vec{\nu}\left|\vec{x}_{\rho}\right|\right) .
\end{aligned}
$$

Now choosing $\vec{\chi}=\left(\varkappa-\frac{\vec{\nu} \cdot \vec{e}_{1}}{\vec{x} \cdot \vec{e}_{1}}\right) \vec{\nu} \in \mathbb{X}=\underline{V}_{\partial_{0}}$ and $\vec{\chi}=\vec{\nu} \in \mathbb{X}=\underline{V}_{\partial_{0}}$ in (3.26), recall (2.10), (3.25) and (3.5a), we see that the two side constraints in (2.43) will be satisfied if $\left(\lambda_{A}(t), \lambda_{V}(t)\right)^{T} \in \mathbb{R}^{2}$ solve the symmetric system

$$
\begin{gathered}
2 \pi\left(\begin{array}{cc}
\left(\vec{x} \cdot \vec{e}_{1},\left(\varkappa-\frac{\vec{\nu} \cdot \vec{e}_{1}}{\vec{x} \cdot \vec{e}_{1}}\right)^{2}\left|\vec{x}_{\rho}\right|\right) & \left(\vec{x} \cdot \vec{e}_{1},\left(\varkappa-\frac{\vec{\nu} \cdot \vec{e}_{1}}{\vec{x} \cdot \vec{e}_{1}}\right)\left|\vec{x}_{\rho}\right|\right) \\
\left(\vec{x} \cdot \vec{e}_{1},\left(\varkappa-\frac{\vec{\nu} \cdot \vec{e}_{1}}{\vec{x} \cdot \vec{e}_{1}}\right)\left|\vec{x}_{\rho}\right|\right) & \left(\vec{x} \cdot \vec{e}_{1},\left|\vec{x}_{\rho}\right|\right)
\end{array}\right)\left(\begin{array}{l}
\lambda_{A} \\
\lambda_{V}
\end{array}\right) \\
=\left(\begin{array}{c}
\left.\left(\vec{y}_{\rho} \cdot \vec{\nu},\left[\left(\varkappa-\frac{\vec{\nu} \cdot \vec{e}_{1}}{\vec{x} \cdot \vec{e}_{1}}\right) \vec{\nu}\right]_{\rho} \cdot \vec{\nu}\left|\vec{x}_{\rho}\right|^{-1}\right)+\left(\vec{f},\left(\varkappa-\frac{\vec{\nu} \cdot \vec{e}_{1}}{\vec{x} \cdot \vec{e}_{1}}\right) \vec{\nu}\left|\vec{x}_{\rho}\right|\right)\right) . \\
\left(\vec{f}, \vec{\nu}\left|\vec{x}_{\rho}\right|\right)
\end{array}\right) .
\end{gathered}
$$

The matrix in (3.28) is symmetric positive semidefinite, and it is singular if and only if $\varkappa_{\mathcal{S}}=\varkappa-\frac{\vec{\nu} \cdot \vec{e}_{1}}{\vec{x} \cdot \vec{e}_{1}}$ is a constant. It can be shown that this is equivalent to $\mathcal{S}(t)$ being a sphere and hence to $\Gamma(t)$ being an open halfcircle. Choosing $\vec{\chi}=\vec{x}_{t} \in \mathbb{X}=\underline{V}_{\partial_{0}}$ in (3.26) and noting (2.43) proves the stability result (3.20) for the weak formulation of the conserved flow, on recalling (3.17), (3.19) and (3.22a).

An alternative formulation of the two conservation side constraints can be obtained by observing that (2.41) is equivalent to

$$
\mathcal{H}^{2}(\mathcal{S}(t))=\mathcal{H}^{2}(\mathcal{S}(0)), \quad \mathcal{L}^{3}(\Omega(t))=\mathcal{L}^{3}(\Omega(0)) .
$$

In order to formulate $(2.41)$ in terms of $\vec{x}$, we define, on recalling (2.38),

$$
A(\vec{x}(t))=2 \pi\left(\vec{x} \cdot \vec{e}_{1},\left|\vec{x}_{\rho}\right|\right)=\mathcal{H}^{2}(\mathcal{S}(t))
$$

and, see e.g. (3.10) of [7],

$$
V(\vec{x}(t))=\pi\left(\left(\vec{x} \cdot \vec{e}_{1}\right)^{2}, \vec{\nu} \cdot \vec{e}_{1}\left|\vec{x}_{\rho}\right|\right)=\mathcal{L}^{3}(\Omega(t)) .
$$

Hence $\left(\lambda_{A}(t), \lambda_{V}(t)\right)^{T} \in \mathbb{R}^{2}$ in (3.27) will be such that (2.43) holds, which is equivalent to

$$
A(\vec{x}(t))=A(\vec{x}(0)), \quad V(\vec{x}(t))=V(\vec{x}(0)) .
$$

Our discretization in Section 3.1.1 will be based on (3.27) and (3.31).

\subsection{Based on $\varkappa_{\mathcal{S}}$}

A drawback of the formulation used in Section 3.1 is that in the case $\partial_{0} I \neq \emptyset$ the integrals featuring the singular fraction $\frac{\vec{\nu} \cdot \vec{e}_{1}}{\vec{x} \cdot \vec{e}_{1}}$ in the Lagrangian (3.10) are not well-defined on the discrete level, and so an appropriate interpretation of these terms is needed. An alternative is to use the mean curvature of the surface as a variable in the weak formulation, i.e. to use a formulation that features (3.9). Then the discretization follows naturally, and a similar approach has been followed by the present authors for flows in Riemannian manifolds in [8].

On recalling $(2.36),(2.22),(3.9)$ and that $\vec{\mu}=\vec{m}$ on $\partial_{M} I$, we define the Lagrangian

$$
\begin{aligned}
\mathcal{L}_{\mathcal{S}}\left(\vec{x}, \varkappa_{\mathcal{S}}^{\star}, \vec{m}, \vec{y}_{\mathcal{S}}\right)= & \pi\left(\alpha\left[\varkappa_{\mathcal{S}}^{\star}-\bar{x}\right]^{2}+2 \lambda, \vec{x} \cdot \vec{e}_{1}\left|\vec{x}_{\rho}\right|\right)+\frac{\beta}{2}\left[2 \pi\left(\vec{x} \cdot \vec{e}_{1} \varkappa_{\mathcal{S}}^{\star},\left|\vec{x}_{\rho}\right|\right)-M_{0}\right]^{2}-\left(\vec{x} \cdot \vec{e}_{1} \varkappa_{\mathcal{S}}^{\star} \vec{\nu}+\vec{e}_{1}, \vec{y}_{\mathcal{S}}\left|\vec{x}_{\rho}\right|\right) \\
& -\left(\left(\vec{x} \cdot \vec{e}_{1}\right) \vec{\tau},\left(\vec{y}_{\mathcal{S}}\right)_{\rho}\right)+2 \pi \varsigma \sum_{p \in \partial_{2} I \cup \partial_{F} I} \vec{x}(p) \cdot \vec{e}_{1}+\sum_{p \in \partial_{C} I}\left[\left(\vec{x} \cdot \vec{e}_{1}\right) \vec{\zeta} \cdot \vec{y}_{\mathcal{S}}\right](p)
\end{aligned}
$$


848

J.W. BARRETT ET AL.

$$
+\sum_{p \in \partial_{M} I}\left[\vec{m} \cdot\left(\left(\vec{x} \cdot \vec{e}_{1}\right) \vec{y}_{\mathcal{S}}-2 \pi \alpha_{G} \vec{e}_{1}\right)\right](p)
$$

for $\vec{x} \in \underline{V}_{\partial_{0}}, \varkappa_{\mathcal{S}}^{\star} \in L^{2}(I), \vec{m}: \partial_{M} I \rightarrow \mathbb{R}^{2}$ and $\vec{y}_{\mathcal{S}} \in \underline{V}_{\partial_{0}}$.

Taking variations $\vec{\eta} \in \underline{V}_{\partial_{0}}$ in $\vec{y}_{\mathcal{S}}$, and setting $\left[\frac{\delta}{\delta \vec{y}_{\mathcal{S}}} \mathcal{L}_{\mathcal{S}}\right](\vec{\eta})=0$, we obtain

$$
\left(\vec{x} \cdot \vec{e}_{1} \varkappa_{\mathcal{S}}^{\star} \vec{\nu}+\vec{e}_{1}, \vec{\eta}\left|\vec{x}_{\rho}\right|\right)+\left(\left(\vec{x} \cdot \vec{e}_{1}\right) \vec{\tau}, \vec{\eta}_{\rho}\right)=\sum_{p \in \partial_{C} I}\left[\left(\vec{x} \cdot \vec{e}_{1}\right) \vec{\zeta} \cdot \vec{\eta}\right](p)+\sum_{p \in \partial_{M} I}\left[\left(\vec{x} \cdot \vec{e}_{1}\right) \vec{m} \cdot \vec{\eta}\right](p) \quad \forall \vec{\eta} \in \underline{V}_{\partial_{0}},
$$

and so combining with (3.9) yields that $\varkappa_{\mathcal{S}}^{\star}=\varkappa_{\mathcal{S}}$. We are going to use this identity from now on. Taking variations $\chi \in L^{2}(I)$ in $\varkappa_{\mathcal{S}}^{\star}$, and setting $\left[\frac{\delta}{\delta \varkappa_{\mathcal{S}}^{\star}} \mathcal{L}_{\mathcal{S}}\right](\chi)=0$, we obtain, on using $\varkappa_{\mathcal{S}}^{\star}=\varkappa_{\mathcal{S}}$, that

$$
2 \pi\left(\alpha\left(\varkappa_{\mathcal{S}}-\bar{\varkappa}\right)+\beta \mathcal{A}_{\mathcal{S}}, \vec{x} \cdot \vec{e}_{1} \chi\left|\vec{x}_{\rho}\right|\right)-\left(\left(\vec{x} \cdot \vec{e}_{1}\right) \vec{y}_{\mathcal{S}}, \chi \vec{\nu}\left|\vec{x}_{\rho}\right|\right)=0 \quad \forall \chi \in L^{2}(I),
$$

where

$$
\mathcal{A}_{\mathcal{S}}(t)=2 \pi\left(\varkappa_{\mathcal{S}}, \vec{x} \cdot \vec{e}_{1}\left|\vec{x}_{\rho}\right|\right)-M_{0} .
$$

We note that

$$
\left[\frac{\delta}{\delta \vec{x}} \mathcal{A}_{\mathcal{S}}\right](\vec{\chi})=2 \pi\left(\varkappa_{\mathcal{S}},\left[\frac{\delta}{\delta \vec{x}} \vec{x} \cdot \vec{e}_{1}\left|\vec{x}_{\rho}\right|\right](\vec{\chi})\right) \quad \forall \vec{\chi} \in \mathbb{X} .
$$

Taking variations in $\vec{m}$, and setting them to zero, yields that

$$
\left(\vec{x} \cdot \vec{e}_{1}\right) \vec{y}_{\mathcal{S}}=2 \pi \alpha_{G} \vec{e}_{1} \quad \text { on } \quad \partial_{M} I .
$$

Taking variations $\vec{\chi} \in \mathbb{X}$ in $\vec{x}$, and setting $2 \pi\left(\left(\vec{x} \cdot \vec{e}_{1}\right) \vec{x}_{t} \cdot \vec{\nu}, \vec{\chi} \cdot \vec{\nu}\left|\vec{x}_{\rho}\right|\right)=-\left[\frac{\delta}{\delta \vec{x}} \mathcal{L}_{\mathcal{S}}\right](\vec{\chi})$ we obtain, on noting (3.36), (3.5b) and (2.37), that

$$
\begin{aligned}
2 \pi\left(\left(\vec{x} \cdot \vec{e}_{1}\right) \vec{x}_{t} \cdot \vec{\nu}, \vec{\chi} \cdot \vec{\nu}\left|\vec{x}_{\rho}\right|\right)= & -\pi\left(\alpha\left[\varkappa_{\mathcal{S}}-\bar{\varkappa}\right]^{2}+2 \lambda+2 \beta \mathcal{A}_{\mathcal{S}} \varkappa_{\mathcal{S}},\left[\frac{\delta}{\delta \vec{x}} \vec{x} \cdot \vec{e}_{1}\left|\vec{x}_{\rho}\right|\right](\vec{\chi})\right) \\
& +\left(\varkappa_{\mathcal{S}} \vec{y}_{\mathcal{S}},\left[\frac{\delta}{\delta \vec{x}}\left(\vec{x} \cdot \vec{e}_{1}\right) \vec{\nu}\left|\vec{x}_{\rho}\right|\right](\vec{\chi})\right)+\left(\vec{e}_{1} \cdot \vec{y}_{\mathcal{S}},\left[\frac{\delta}{\delta \vec{x}}\left|\vec{x}_{\rho}\right|\right](\vec{\chi})\right) \\
& +\left(\left(\vec{y}_{\mathcal{S}}\right)_{\rho},\left[\frac{\delta}{\delta \vec{x}}\left(\vec{x} \cdot \vec{e}_{1}\right) \vec{\tau}\right](\vec{\chi})\right)-\sum_{p \in \partial_{2} I \cup \partial_{F} I}\left[\left[2 \pi \varsigma+\vec{m} \cdot \vec{y}_{\mathcal{S}}\right] \vec{\chi} \cdot \vec{e}_{1}\right](p) \quad \forall \vec{\chi} \in \mathbb{X} .
\end{aligned}
$$

Choosing $\vec{\chi}=\vec{x}_{t} \in \mathbb{X}$ in (3.38), and recalling (3.4), yields

$$
\begin{aligned}
2 \pi\left(\vec{x} \cdot \vec{e}_{1}\left(\vec{x}_{t} \cdot \vec{\nu}\right)^{2},\left|\vec{x}_{\rho}\right|\right)= & -\pi\left(\alpha\left[\varkappa_{\mathcal{S}}-\bar{\varkappa}\right]^{2}+2 \lambda+2 \beta \mathcal{A}_{\mathcal{S}} \varkappa_{\mathcal{S}},\left[\vec{x} \cdot \vec{e}_{1}\left|\vec{x}_{\rho}\right|\right]_{t}\right)+\left(\varkappa_{\mathcal{S}} \vec{y}_{\mathcal{S}},\left[\left(\vec{x} \cdot \vec{e}_{1}\right) \vec{\nu}\left|\vec{x}_{\rho}\right|\right]_{t}\right) \\
& +\left(\vec{e}_{1} \cdot \vec{y}_{\mathcal{S}},\left[\left|\vec{x}_{\rho}\right|\right]_{t}\right)+\left(\left(\vec{y}_{\mathcal{S}}\right)_{\rho},\left[\left(\vec{x} \cdot \vec{e}_{1}\right) \vec{\tau}\right]_{t}\right)-\sum_{p \in \partial_{2} I \cup \partial_{F} I}\left[\left[2 \pi \varsigma+\vec{m} \cdot \vec{y}_{\mathcal{S}}\right] \vec{x}_{t} \cdot \vec{e}_{1}\right](p) .
\end{aligned}
$$

Differentiating (3.33) with respect to $t$ and then choosing $\vec{\eta}=\vec{y}_{\mathcal{S}} \in \underline{V}_{\partial_{0}}$, on recalling $\varkappa_{\mathcal{S}}^{\star}=\varkappa_{\mathcal{S}}, \vec{x}_{t} \in \mathbb{X}$ and $\vec{\zeta}$ is independent of $t$ and so the term on $\partial_{C} I$ vanishes, yields that

$$
\begin{gathered}
\left(\left(\varkappa_{\mathcal{S}}\right)_{t},\left(\vec{x} \cdot \vec{e}_{1}\right) \vec{y}_{\mathcal{S}} \cdot \vec{\nu}\left|\vec{x}_{\rho}\right|\right)+\left(\varkappa_{\mathcal{S}} \vec{y}_{\mathcal{S}},\left[\vec{x} \cdot \vec{e}_{1}\left|\vec{x}_{\rho}\right| \vec{\nu}\right]_{t}\right)+\left(\vec{e}_{1}, \vec{y}_{\mathcal{S}}\left[\left|\vec{x}_{\rho}\right|\right]_{t}\right)+\left(\left[\left(\vec{x} \cdot \vec{e}_{1}\right) \vec{\tau}\right]_{t},\left(\vec{y}_{\mathcal{S}}\right)_{\rho}\right) \\
=\sum_{p \in \partial_{M} I}\left[\left(\vec{x}_{t} \cdot \vec{e}_{1}\right) \vec{m} \cdot \vec{y}_{\mathcal{S}}+\left(\vec{x} \cdot \vec{e}_{1}\right) \vec{m}_{t} \cdot \vec{y}_{\mathcal{S}}\right](p) .
\end{gathered}
$$


It follows from (3.40), (3.37) and (3.34) with $\chi=\left[\varkappa_{\mathcal{S}}\right]_{t}$ that

$$
\begin{aligned}
& \left(\varkappa_{\mathcal{S}} \vec{y}_{\mathcal{S}},\left[\left(\vec{x} \cdot \vec{e}_{1}\right) \vec{\nu}\left|\vec{x}_{\rho}\right|\right]_{t}\right)+\left(\vec{e}_{1} \cdot \vec{y}_{\mathcal{S}},\left[\left|\vec{x}_{\rho}\right|\right]_{t}\right)+\left(\left(\vec{y}_{\mathcal{S}}\right)_{\rho},\left[\left(\vec{x} \cdot \vec{e}_{1}\right) \vec{\tau}\right]_{t}\right) \\
& \quad=-\left(\left[\varkappa_{\mathcal{S}}\right]_{t},\left(\vec{x} \cdot \vec{e}_{1}\right) \vec{y}_{\mathcal{S}} \cdot \vec{\nu}\left|\vec{x}_{\rho}\right|\right)+\sum_{p \in \partial_{M} I}\left[\left(\vec{x}_{t} \cdot \vec{e}_{1}\right) \vec{m} \cdot \vec{y}_{\mathcal{S}}+2 \pi \alpha_{G} \vec{m}_{t} \cdot \vec{e}_{1}\right](p) \\
& \quad=-2 \pi\left(\alpha\left(\varkappa_{\mathcal{S}}-\bar{\varkappa}\right)+\beta \mathcal{A}_{\mathcal{S}}, \vec{x} \cdot \vec{e}_{1}\left[\varkappa_{\mathcal{S}}\right]_{t}\left|\vec{x}_{\rho}\right|\right)+\sum_{p \in \partial_{M} I}\left[\left(\vec{x}_{t} \cdot \vec{e}_{1}\right) \vec{m} \cdot \vec{y}_{\mathcal{S}}+2 \pi \alpha_{G} \vec{m}_{t} \cdot \vec{e}_{1}\right](p) .
\end{aligned}
$$

Combining (3.39) and (3.41) yields that

$$
\begin{aligned}
2 \pi\left(\vec{x} \cdot \vec{e}_{1}\left(\vec{x}_{t} \cdot \vec{\nu}\right)^{2},\left|\vec{x}_{\rho}\right|\right)= & -\pi\left(\alpha\left[\varkappa_{\mathcal{S}}-\bar{\varkappa}\right]^{2}+2 \lambda+2 \beta \mathcal{A}_{\mathcal{S}} \varkappa_{\mathcal{S}},\left[\vec{x} \cdot \vec{e}_{1}\left|\vec{x}_{\rho}\right|\right]_{t}\right) \\
& -2 \pi\left(\alpha\left(\varkappa_{\mathcal{S}}-\bar{\varkappa}\right)+\beta \mathcal{A}_{\mathcal{S}}, \vec{x} \cdot \vec{e}_{1}\left[\varkappa_{\mathcal{S}}\right]_{t}\left|\vec{x}_{\rho}\right|\right)-2 \pi \varsigma \sum_{p \in \partial_{2} I \cup \partial_{F} I} \vec{x}_{t}(p) \cdot \vec{e}_{1} \\
& +2 \pi \alpha_{G} \sum_{p \in \partial_{M} I} \vec{m}_{t}(p) \cdot \vec{e}_{1} \\
= & -\pi \frac{\mathrm{d}}{\mathrm{d} t}\left(\alpha\left[\varkappa_{\mathcal{S}}-\bar{\varkappa}\right]^{2}+2 \lambda, \vec{x} \cdot \vec{e}_{1}\left|\vec{x}_{\rho}\right|\right)-\frac{\beta}{2} \frac{\mathrm{d}}{\mathrm{d} t} \mathcal{A}_{\mathcal{S}}^{2}-2 \pi \varsigma \sum_{p \in \partial_{2} I \cup \partial_{F} I} \vec{x}_{t}(p) \cdot \vec{e}_{1} \\
& +2 \pi \alpha_{G} \sum_{p \in \partial_{M} I} \vec{m}_{t}(p) \cdot \vec{e}_{1} \\
= & -\frac{\mathrm{d}}{\mathrm{d} t} \widehat{E}(t),
\end{aligned}
$$

where we have recalled the definition (2.36). Of course, Remark 3.1 also applies to (3.42).

In order to derive a suitable weak formulation, we now return to (3.38). Using (3.3) and noting (2.3), (3.38) can be rewritten as

$$
\begin{aligned}
2 \pi\left(\left(\vec{x} \cdot \vec{e}_{1}\right) \vec{x}_{t} \cdot \vec{\nu}, \vec{\chi} \cdot \vec{\nu}\left|\vec{x}_{\rho}\right|\right)= & -\left(\pi \vec{x} \cdot \vec{e}_{1}\left[\alpha\left(\varkappa_{\mathcal{S}}-\bar{\varkappa}\right)^{2}+2 \lambda+2 \beta \mathcal{A}_{\mathcal{S}} \varkappa_{\mathcal{S}}\right]-\vec{y}_{\mathcal{S}} \cdot \vec{e}_{1}, \vec{\chi}_{\rho} \cdot \vec{\tau}\right) \\
& -\left(\left[\pi\left[\alpha\left(\varkappa_{\mathcal{S}}-\bar{\varkappa}\right)^{2}+2 \lambda+2 \beta \mathcal{A}_{\mathcal{S}} \varkappa_{\mathcal{S}}\right]-\varkappa_{\mathcal{S}} \vec{y}_{\mathcal{S}} \cdot \vec{\nu}\right]\left|\vec{x}_{\rho}\right|-\left(\vec{y}_{\mathcal{S}}\right)_{\rho} \cdot \vec{\tau}, \vec{\chi} \cdot \vec{e}_{1}\right) \\
& -\left(\vec{x} \cdot \vec{e}_{1} \varkappa_{\mathcal{S}} \vec{y}_{\mathcal{S}}, \vec{\chi}_{\rho}^{\perp}\right)+\left(\left(\vec{x} \cdot \vec{e}_{1}\right)\left(\vec{y}_{\mathcal{S}}\right)_{\rho} \cdot \vec{\nu}, \vec{\chi}_{\rho} \cdot \vec{\nu}\left|\vec{x}_{\rho}\right|^{-1}\right) \\
& -\sum_{p \in \partial_{2} I \cup \partial_{F} I}\left[\left[2 \pi \varsigma+\vec{m} \cdot \vec{y}_{\mathcal{S}}\right] \vec{\chi} \cdot \vec{e}_{1}\right](p) \quad \forall \vec{\chi} \in \mathbb{X} .
\end{aligned}
$$

Overall, we obtain the following weak formulation from (3.43), (3.34), (3.33), (3.37) and (3.35), on recalling $\varkappa_{\mathcal{S}}^{\star}=\varkappa_{\mathcal{S}},(3.1 \mathrm{a})$ and $(2.3)$.

$\left(\mathcal{P}_{\mathcal{S}}\right)$ Let $\vec{x}(\cdot, 0) \in \underline{V}_{\partial_{0}}$ and $\alpha \in \mathbb{R}_{>0}, \bar{\varkappa}, M_{0}, \alpha_{G}, \lambda, \varsigma \in \mathbb{R}, \beta \in \mathbb{R}_{\geq 0}, \vec{\zeta}: \partial_{C} I \rightarrow \mathbb{S}^{1}$ be given. For $t \in(0, T]$, find $\vec{x}(\cdot, t) \in \underline{V}_{\partial_{0}}$, with $\vec{x}_{t}(\cdot, t) \in \mathbb{X}, \varkappa_{\mathcal{S}}(\cdot, t) \in L^{2}(I), \vec{y}_{\mathcal{S}}(\cdot, t) \in \underline{V}_{\partial_{0}}$, with $\left[\left(\vec{x} \cdot \vec{e}_{1}\right) \vec{y}_{\mathcal{S}}\right](\cdot, t) \in \mathbb{Y}\left(2 \pi \alpha_{G} \vec{e}_{1}\right)$, and $\vec{m}(\cdot, t): \partial_{M} I \rightarrow \mathbb{R}^{2}$ such that

$$
\begin{aligned}
& 2 \pi\left(\left(\vec{x} \cdot \vec{e}_{1}\right) \vec{x}_{t} \cdot \vec{\nu}, \vec{\chi} \cdot \vec{\nu}\left|\vec{x}_{\rho}\right|\right)-\left(\left(\vec{x} \cdot \vec{e}_{1}\right)\left(\vec{y}_{\mathcal{S}}\right)_{\rho} \cdot \vec{\nu}, \vec{\chi}_{\rho} \cdot \vec{\nu}\left|\vec{x}_{\rho}\right|^{-1}\right) \\
&=-\left(\pi \vec{x} \cdot \vec{e}_{1}\left[\alpha\left(\varkappa_{\mathcal{S}}-\bar{\varkappa}\right)^{2}+2 \lambda+2 \beta \mathcal{A}_{\mathcal{S}} \varkappa_{\mathcal{S}}\right]-\vec{y}_{\mathcal{S}} \cdot \vec{e}_{1}, \vec{\chi}_{\rho} \cdot \vec{\tau}\right) \\
&-\left(\left[\pi\left[\alpha\left(\varkappa_{\mathcal{S}}-\bar{\varkappa}\right)^{2}+2 \lambda+2 \beta \mathcal{A}_{\mathcal{S}} \varkappa_{\mathcal{S}}\right]-\varkappa_{\mathcal{S}} \vec{y}_{\mathcal{S}} \cdot \vec{\nu}\right]\left|\vec{x}_{\rho}\right|-\left(\vec{y}_{\mathcal{S}}\right)_{\rho} \cdot \vec{\tau}, \vec{\chi} \cdot \vec{e}_{1}\right) \\
&+\left(\vec{x} \cdot \vec{e}_{1} \varkappa_{\mathcal{S}} \vec{y}_{\mathcal{S}}^{\perp}, \vec{\chi}_{\rho}\right)-\sum_{p \in \partial_{2} I \cup \partial_{F} I}\left[\left[2 \pi \varsigma+\vec{m} \cdot \vec{y}_{\mathcal{S}}\right] \vec{\chi} \cdot \vec{e}_{1}\right](p) \quad \forall \vec{\chi} \in \mathbb{X}, \\
& 2 \pi\left(\vec{x} \cdot \vec{e}_{1}\left[\alpha\left(\varkappa_{\mathcal{S}}-\bar{\varkappa}\right)+\beta \mathcal{A}_{\mathcal{S}}\right], \chi\left|\vec{x}_{\rho}\right|\right)-\left(\left(\vec{x} \cdot \vec{e}_{1}\right) \vec{y}_{\mathcal{S}}, \chi \vec{\nu}\left|\vec{x}_{\rho}\right|\right)=0 \quad \forall \chi \in L^{2}(I),
\end{aligned}
$$




$$
\left(\vec{x} \cdot \vec{e}_{1} \varkappa_{\mathcal{S}} \vec{\nu}+\vec{e}_{1}, \vec{\eta}\left|\vec{x}_{\rho}\right|\right)+\left(\left(\vec{x} \cdot \vec{e}_{1}\right) \vec{x}_{\rho}, \vec{\eta}_{\rho}\left|\vec{x}_{\rho}\right|^{-1}\right)=\sum_{p \in \partial_{C} I}\left[\left(\vec{x} \cdot \vec{e}_{1}\right) \vec{\zeta} \cdot \vec{\eta}\right](p)+\sum_{p \in \partial_{M} I}\left[\left(\vec{x} \cdot \vec{e} \vec{m}_{1}\right) \vec{m} \cdot \vec{\eta}\right](p) \quad \forall \vec{\eta} \in \underline{V}_{\partial_{0}},
$$

where $\mathcal{A}_{\mathcal{S}}(t)$ is given by (3.35). Similarly to (3.22), we note that the number of unknowns fixed via $\left(\vec{x} \cdot \vec{e}_{1}\right) \vec{y}_{\mathcal{S}} \in$ $\mathbb{Y}\left(2 \pi \alpha_{G} \vec{e}_{1}\right)$ on $\partial_{M} I$ is matched by the new degrees of freedom arising from $\{\vec{m}(p)\}_{p \in \partial_{M} I}$.

Similarly to (3.23) and (3.24), one can show that (3.44) is independent of the tangential part $\vec{y}_{\mathcal{S}} \cdot \vec{\tau}$ of $\vec{y}_{\mathcal{S}}$. We refer to Appendix A.2, where we show that (3.44) for a sufficiently smooth solution gives rise to the strong form (2.24) and (2.9).

Remark 3.2. Similarly to the procedure in Section 3.1.1, we can state a weak formulation for the conserving flow (2.42). In particular, on writing (3.44a) as

$$
2 \pi\left(\left(\vec{x} \cdot \vec{e}_{1}\right) \vec{x}_{t} \cdot \vec{\nu}, \vec{\chi} \cdot \vec{\nu}\left|\vec{x}_{\rho}\right|\right)-\left(\left(\vec{x} \cdot \vec{e}_{1}\right)\left(\vec{y}_{\mathcal{S}}\right)_{\rho} \cdot \vec{\nu}, \vec{\chi}_{\rho} \cdot \vec{\nu}\left|\vec{x}_{\rho}\right|^{-1}\right)=\left(\vec{f}_{\mathcal{S}}, \vec{\chi}\left|\vec{x}_{\rho}\right|\right) \quad \forall \vec{\chi} \in \mathbb{X},
$$

we can formulate the conserving flow as

$$
\begin{aligned}
& 2 \pi\left(\left(\vec{x} \cdot \vec{e}_{1}\right) \vec{x}_{t} \cdot \vec{\nu}, \vec{\chi} \cdot \vec{\nu}\left|\vec{x}_{\rho}\right|\right)-\left(\left(\vec{x} \cdot \vec{e}_{1}\right)\left(\vec{y}_{\mathcal{S}}\right)_{\rho} \cdot \vec{\nu}, \vec{\chi}_{\rho} \cdot \vec{\nu}\left|\vec{x}_{\rho}\right|^{-1}\right)=\left(\vec{f}_{\mathcal{S}}, \vec{\chi}\left|\vec{x}_{\rho}\right|\right) \\
& \quad-2 \pi \lambda_{A}\left[\left(\vec{e}_{1}, \vec{\chi}\left|\vec{x}_{\rho}\right|\right)+\left(\left(\vec{x} \cdot \vec{e}_{1}\right) \vec{\tau}, \vec{\chi}_{\rho}\right)\right]-2 \pi \lambda_{V}\left(\left(\vec{x} \cdot \vec{e}_{1}\right) \vec{\nu}, \vec{\chi}\left|\vec{x}_{\rho}\right|\right) \quad \forall \vec{\chi} \in \mathbb{X},
\end{aligned}
$$

where $\left(\lambda_{A}(t), \lambda_{V}(t)\right)^{T} \in \mathbb{R}^{2}$ are chosen such that (2.43) holds.

\section{SEMIDISCRETE SCHEMES}

Let $[0,1]=\bigcup_{j=1}^{J} I_{j}, J \geq 3$, be a decomposition of $[0,1]$ into intervals given by the nodes $q_{j}, I_{j}=\left[q_{j-1}, q_{j}\right]$. For simplicity, and without loss of generality, we assume that the subintervals form an equipartitioning of $[0,1]$, i.e. that

$$
q_{j}=j h, \quad \text { with } \quad h=J^{-1}, \quad j=0, \ldots, J .
$$

Clearly, if $I=\mathbb{R} / \mathbb{Z}$ we identify $0=q_{0}=q_{J}=1$. In addition, we let $q_{J+1}=q_{1}$.

The necessary finite element spaces are defined as follows:

$$
V^{h}=\left\{\chi \in C^{0}(\bar{I}):\left.\chi\right|_{I_{j}} \text { is linear, } j=1, \ldots, J\right\}, V_{0}^{h}=H_{0}^{1}(I) \cap V^{h} \quad \text { and } \quad \underline{V}^{h}=\left[V^{h}\right]^{2}, \quad \underline{V}_{0}^{h}=\left[V_{0}^{h}\right]^{2} .
$$

In addition, we define $\underline{V}_{\partial_{0}}^{h}=\underline{V}^{h} \cap \underline{V}_{\partial_{0}}, W^{h}=V^{h}, W_{\partial_{0}}^{h}=\left\{\eta \in V^{h}: \eta(\rho)=0 \forall \rho \in \partial_{0} I\right\}, \mathbb{X}^{h}=\mathbb{X} \cap \underline{V}^{h}$ and, for a given $\vec{z} \in \mathbb{R}^{2}, \mathbb{Y}^{h}(\vec{z})=\mathbb{Y}(\vec{z}) \cap \underline{V}^{h}$. Let $\left\{\chi_{j}\right\}_{j=j_{0}}^{J}$ denote the standard basis of $V^{h}$, where $j_{0}=0$ if $I=(0,1)$ and $j_{0}=1$ if $I=\mathbb{R} / \mathbb{Z}$. We also set $j_{1}=J-1$ if $I=(0,1)$ and $j_{1}=J$ if $I=\mathbb{R} / \mathbb{Z}$. For later use, we let $\pi^{h}: C^{0}(\bar{I}) \rightarrow V^{h}$ be the standard interpolation operator at the nodes $\left\{q_{j}\right\}_{j=0}^{J}$, and similarly $\pi_{\partial_{0}}^{h}: C^{0}(\bar{I}) \rightarrow W_{\partial_{0}}^{h}$, as well as $\vec{\pi}^{h}:\left[C^{0}(\bar{I})\right]^{2} \rightarrow \underline{V}^{h}$.

Let $(\cdot, \cdot)$ denote the $L^{2}$-inner product on $I$, and define the mass lumped $L^{2}$-inner product $(f, g)^{h}$, for two piecewise continuous functions, with possible jumps at the nodes $\left\{q_{j}\right\}_{j=1}^{J}$, via

$$
(f, g)^{h}=\frac{1}{2} h \sum_{j=1}^{J}\left[(f g)\left(q_{j}^{-}\right)+(f g)\left(q_{j-1}^{+}\right)\right],
$$

where we define $f\left(q_{j}^{ \pm}\right)=\lim _{\delta \searrow 0} f\left(q_{j} \pm \delta\right)$. The definition (4.2) naturally extends to vector valued functions.

Let $\left(\vec{X}^{h}(t)\right)_{t \in[0, T]}$, with $\vec{X}^{h}(\cdot, t) \in \underline{V}_{\partial_{0}}^{h}$, be an approximation to $(\vec{x}(t))_{t \in[0, T]}$ and define $\Gamma^{h}(t)=\vec{X}^{h}(\bar{I}, t)$. A natural discrete analogue of the well-posedness assumptions for the continuous solution $\vec{x}$ is given as follows. 
Assumption 4.1. Let

$$
\vec{X}^{h}(\rho, t) \cdot \vec{e}_{1}>0 \quad \forall \rho \in \bar{I} \backslash \partial_{0} I, t \in[0, T] .
$$

In addition, let $\vec{X}^{h}\left(q_{j}, t\right) \neq \vec{X}^{h}\left(q_{j+1}, t\right), j=0, \ldots, J-1$, for all $t \in[0, T]$.

Then, similarly to $(2.3)$, we set

$$
\vec{\tau}^{h}=\vec{X}_{s}^{h}=\frac{\vec{X}_{\rho}^{h}}{\left|\vec{X}_{\rho}^{h}\right|} \quad \text { and } \quad \vec{\nu}^{h}=-\left(\vec{\tau}^{h}\right)^{\perp} \quad \text { in } \bar{I},
$$

which is well-defined if Assumption 4.1 holds. We note that (4.3) implies $\vec{\tau}^{h} \cdot \vec{e}_{1} \neq 0$ on elements touching the $x_{2}$-axis, and so

$$
\vec{\nu}^{h} \cdot \vec{e}_{2} \neq 0 \quad \text { on } \partial_{0} I
$$

compare also with (2.10) and (2.11).

Assumption 4.2. Let Assumption 4.1 hold and let $\vec{X}^{h}\left(q_{j-1}, t\right) \neq \vec{X}^{h}\left(q_{j+1}, t\right), j=1, \ldots, j_{1}$, for all $t \in[0, T]$.

We note that Assumption 4.2 is only violated if two neighbouring elements of $\Gamma^{h}(t)$ lie identically on top of each other. For our fully discrete schemes, this never happens in practice. For later use, we let $\vec{\omega}^{h} \in \underline{V}^{h}$ be the mass-lumped $L^{2}$-projection of $\vec{\nu}^{h}$ onto $\underline{V}^{h}$, i.e.

$$
\left(\vec{\omega}^{h}, \vec{\varphi}\left|\vec{X}_{\rho}^{h}\right|\right)^{h}=\left(\vec{\nu}^{h}, \vec{\varphi}\left|\vec{X}_{\rho}^{h}\right|\right)=\left(\vec{\nu}^{h}, \vec{\varphi}\left|\vec{X}_{\rho}^{h}\right|\right)^{h} \quad \forall \vec{\varphi} \in \underline{V}^{h} .
$$

Combining (4.5), (4.2) and (4.4) yields that

$$
\vec{\omega}^{h}\left(q_{j}\right)= \begin{cases}-\frac{\left(\vec{X}^{h}\left(q_{j+1}\right)-\vec{X}^{h}\left(q_{j-1}\right)\right)^{\perp}}{\left|\vec{X}^{h}\left(q_{j+1}\right)-\vec{X}^{h}\left(q_{j}\right)\right|+\left|\vec{X}^{h}\left(q_{j}\right)-\vec{X}^{h}\left(q_{j-1}\right)\right|} & q_{j} \in \bar{I} \backslash \partial I, \\ \vec{\nu}^{h}\left(q_{j}\right) & q_{j} \in \partial I .\end{cases}
$$

It follows that $\vec{v}^{h} \in \underline{V}^{h}$, defined by

$$
\vec{v}^{h}=\vec{\pi}^{h}\left[\frac{\vec{\omega}^{h}}{\left|\vec{\omega}^{h}\right|}\right],
$$

is well-defined if Assumption 4.2 holds. We also define $\underline{\underline{Q}}^{h} \in\left[V^{h}\right]^{2 \times 2}$ defined by

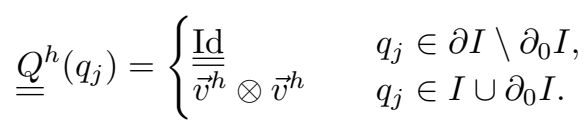

Later on we will describe the evolution of $\Gamma^{h}(t)$ through $\vec{\pi}^{h}\left[\underline{\underline{Q}}^{h} \vec{X}_{t}^{h}\right]$, for $\vec{X}_{t}^{h} \in \mathbb{X}^{h}$. This will allow tangential motion for interior nodes, because we will only let a discrete normal component of $\vec{X}_{t}^{h}$ be specified through an appropriate variation of the discrete energy. But crucially, we will specify the full velocity $\vec{X}_{t}^{h}$ through this energy variation at boundary nodes $q_{j} \in \partial I \backslash \partial_{0} I$. This is because at these boundary nodes we cannot allow an arbitrary tangential motion, as this would affect the evolution of $\Gamma^{h}(t)$ itself, and not just the evolution of its parameterization $\vec{X}^{h}$. A similar strategy has been pursued by the authors in (3.19) of [6].

Similarly to (3.3), on noting (3.2) and (4.4), we have for all $\vec{\chi} \in \mathbb{X}^{h}$ on $I_{j}, j=1, \ldots, J$, that

$$
\left[\frac{\delta}{\delta \vec{X}^{h}}\left|\vec{X}_{\rho}^{h}\right|\right](\vec{\chi})=\frac{\vec{X}_{\rho}^{h} \cdot \vec{\chi}_{\rho}}{\left|\vec{X}_{\rho}^{h}\right|}=\vec{\tau}^{h} \cdot \vec{\chi}_{\rho}
$$




$$
\begin{aligned}
{\left[\frac{\delta}{\delta \vec{X}^{h}} \vec{\tau}^{h}\right](\vec{\chi}) } & =\left[\frac{\delta}{\delta \vec{X}^{h}} \frac{\vec{X}_{\rho}^{h}}{\left|\vec{X}_{\rho}^{h}\right|}\right](\vec{\chi})=\frac{\vec{\chi}_{\rho}}{\left|\vec{X}_{\rho}^{h}\right|}-\frac{\vec{X}_{\rho}^{h}}{\left|\vec{X}_{\rho}^{h}\right|^{2}} \frac{\vec{X}_{\rho}^{h} \cdot \vec{\chi}_{\rho}}{\left|\vec{X}_{\rho}^{h}\right|}=\vec{\chi}_{s}-\vec{\tau}^{h}\left(\vec{\chi}_{s} \cdot \vec{\tau}^{h}\right)=\left(\vec{\chi}_{s} \cdot \vec{\nu}^{h}\right) \vec{\nu}^{h}, \\
{\left[\frac{\delta}{\delta \vec{X}^{h}} \vec{\nu}^{h}\right](\vec{\chi}) } & =-\left[\frac{\delta}{\delta \vec{X}^{h}}\left(\vec{\tau}^{h}\right)^{\perp}\right](\vec{\chi})=-\left(\vec{\chi}_{s} \cdot \vec{\nu}^{h}\right)\left(\vec{\nu}^{h}\right)^{\perp}=-\left(\vec{\chi}_{s} \cdot \vec{\nu}^{h}\right) \vec{\tau}^{h}, \\
{\left[\frac{\delta}{\delta \vec{X}^{h}} \vec{\nu}^{h}\left|\vec{X}_{\rho}^{h}\right|\right](\vec{\chi}) } & =-\left[\frac{\delta}{\delta \vec{X}^{h}}\left(\vec{X}_{\rho}^{h}\right)^{\perp}\right](\vec{\chi})=-\vec{\chi}_{\rho}^{\perp} .
\end{aligned}
$$

In addition to (4.8), we will require $\left[\frac{\delta}{\delta \vec{X}^{h}} \vec{\omega}^{h}\right](\vec{\chi})$. It follows from (4.5), (4.8) and (4.4) that

$$
\begin{aligned}
\left(\left[\frac{\delta}{\delta \vec{X}^{h}} \vec{\omega}^{h}\right](\vec{\chi}), \vec{\varphi}\left|\vec{X}_{\rho}^{h}\right|\right)^{h} & =\left(\left[\frac{\delta}{\delta \vec{X}^{h}} \vec{\nu}^{h}\right](\vec{\chi}), \vec{\varphi}\left|\vec{X}_{\rho}^{h}\right|\right)^{h}-\left(\vec{\omega}^{h}-\vec{\nu}^{h}, \vec{\varphi}\left[\frac{\delta}{\delta \vec{X}^{h}}\left|\vec{X}_{\rho}^{h}\right|\right](\vec{\chi})\right)^{h} \\
& =-\left(\left(\vec{\nu}^{h} \cdot \vec{\chi}_{\rho}\right) \vec{\tau}^{h}, \vec{\varphi}\right)^{h}-\left(\vec{\tau}^{h} \cdot \vec{\chi}_{\rho}\left(\vec{\omega}^{h}-\vec{\nu}^{h}\right), \vec{\varphi}\right)^{h} \quad \forall \vec{\varphi} \in \underline{V}_{\partial_{0}}^{h} .
\end{aligned}
$$

\subsection{Based on $\kappa^{h}$}

As the discrete analogue of (3.8), we let $\vec{X}^{h} \in \underline{V}_{\partial_{0}}^{h}, \kappa^{h} \in V^{h}$ and $\vec{m}^{h}: \partial_{M} I \rightarrow \mathbb{R}^{2}$ be such that

$$
\left(\kappa^{h} \vec{\nu}^{h}, \vec{\eta}\left|\vec{X}_{\rho}^{h}\right|\right)^{h}+\left(\vec{\tau}^{h}, \vec{\eta}_{\rho}\right)=\sum_{p \in \partial_{C} I}[\vec{\zeta} \cdot \vec{\eta}](p)+\sum_{p \in \partial_{M} I}\left[\vec{m}^{h} \cdot \vec{\eta}\right](p) \quad \forall \vec{\eta} \in \underline{V}_{\partial_{0}}^{h},
$$

where we recall (4.4).

We would like to mimic on the discrete level the procedure in Section 3.1. However, a naive discretization of (3.10) will not give a well-defined Lagrangian, since a discrete variant of (2.12) will in general not hold. To overcome the arising singularity in a discretization of (3.10), we now introduce the following discrete approximation of $\varkappa_{\mathcal{S}}$, which will be based on $\kappa^{h}$. In particular, on recalling (2.12) and (4.5), we introduce, given $\vec{X}^{h} \in \underline{V}_{\partial_{0}}^{h}$ and $\kappa^{h} \in V^{h}$, the function $\mathfrak{K}^{h}\left(\vec{X}^{h}, \kappa^{h}\right) \in V^{h}$ such that

$$
\left[\mathfrak{K}^{h}\left(\vec{X}^{h}, \kappa^{h}\right)\right]\left(q_{j}\right)= \begin{cases}\kappa^{h}\left(q_{j}\right)-\frac{\vec{\omega}^{h}\left(q_{j}\right) \cdot \vec{e}_{1}}{\vec{X}^{h}\left(q_{j}\right) \cdot \vec{e}_{1}} & q_{j} \in \bar{I} \backslash \partial_{0} I, \\ 2 \kappa^{h}\left(q_{j}\right) & q_{j} \in \partial_{0} I\end{cases}
$$

Clearly, using $\vec{\nu}^{h}$ in place of $\vec{\omega}^{h}$ in (4.11) would not be well-defined for interior nodes. For later use we also define $\mathfrak{Z}^{h} \in V^{h}$ such that

$$
\mathfrak{Z}^{h}\left(q_{j}\right)= \begin{cases}1 & q_{j} \in \bar{I} \backslash \partial_{0} I \\ 2 & q_{j} \in \partial_{0} I\end{cases}
$$

On noting (4.11), we define the discrete analogue of the energy (2.36)

$$
\begin{aligned}
\widehat{E}^{h}(t)= & \pi\left(\alpha\left[\mathfrak{K}^{h}\left(\vec{X}^{h}, \kappa^{h}\right)-\bar{\varkappa}\right]^{2}+2 \lambda, \vec{X}^{h} \cdot \vec{e}_{1}\left|\vec{X}_{\rho}^{h}\right|\right)^{h}+\frac{\beta}{2}\left(\mathcal{A}^{h}(t)\right)^{2} \\
& -2 \pi \alpha_{G} \sum_{p \in \partial_{M} I} \vec{m}^{h}(p) \cdot \vec{e}_{1}+2 \pi \varsigma \sum_{p \in \partial_{2} I \cup \partial_{F} I} \vec{X}^{h}(p) \cdot \vec{e}_{1}
\end{aligned}
$$

where

$$
\mathcal{A}^{h}(t)=2 \pi\left(\left(\vec{X}^{h} \cdot \vec{e}_{1}\right) \kappa^{h}-\vec{\nu}^{h} \cdot \vec{e}_{1},\left|\vec{X}_{\rho}^{h}\right|\right)^{h}-M_{0}
$$


Remark 4.3. We observe that the energy $\widehat{E}^{h}(t)$ does not depend on the values of $\kappa^{h}$ on $\partial_{0} I$. We will thus fix these values to be zero from now on. A welcome side effect of this procedure is that on assuming that e.g. $0 \in \partial_{0} I$, then choosing $\vec{\eta}=\chi_{0} \vec{e}_{2}$ in (4.10) yields that $\left(\vec{X}^{h}\left(q_{1}\right)-\vec{X}^{h}\left(q_{0}\right)\right) \cdot \vec{e}_{2}=0$. Similarly we get $\left(\vec{X}^{h}\left(q_{J}\right)-\vec{X}^{h}\left(q_{J-1}\right)\right) \cdot \vec{e}_{2}=0$ if $1 \in \partial_{0} I$.

Without fixing $\kappa^{h}$ to be zero on $\partial_{0} I$, we observe numerical difficulties in practice for fully discrete variants of the semidiscrete approximation that we are going to derive.

Similarly to (3.10), we define the discrete Lagrangian

$$
\begin{aligned}
\mathcal{L}^{h}\left(\vec{X}^{h}, \kappa^{h}, \vec{m}^{h}, \vec{Y}^{h}\right)= & \pi\left(\alpha\left[\mathfrak{K}^{h}\left(\vec{X}^{h}, \kappa^{h}\right)-\bar{\varkappa}\right]^{2}+2 \lambda, \vec{X}^{h} \cdot \vec{e}_{1}\left|\vec{X}_{\rho}^{h}\right|\right)^{h}+\frac{\beta}{2}\left[2 \pi \left(\left(\vec{X}^{h} \cdot \vec{e}_{1}\right) \kappa^{h}\right.\right. \\
& \left.\left.-\vec{\nu}^{h} \cdot \vec{e}_{1},\left|\vec{X}_{\rho}^{h}\right|\right)^{h}-M_{0}\right]^{2}-\left(\kappa^{h} \vec{\nu}^{h}, \vec{Y}^{h}\left|\vec{X}_{\rho}^{h}\right|\right)^{h}-\left(\vec{\tau}^{h}, \vec{Y}_{\rho}^{h}\right)+2 \pi \varsigma \sum_{p \in \partial_{2} I \cup \partial_{F} I} \vec{X}^{h}(p) \cdot \vec{e}_{1} \\
& +\sum_{p \in \partial_{C} I}\left[\vec{\zeta} \cdot \vec{Y}^{h}\right](p)+\sum_{p \in \partial_{M} I}\left[\vec{m}^{h} \cdot\left(\vec{Y}^{h}-2 \pi \alpha_{G} \vec{e}_{1}\right)\right](p),
\end{aligned}
$$

for the minimization of the energy (4.13) subject to the side constraint (4.10), where $\vec{X}^{h} \in \underline{V}_{\partial_{0}}^{h}, \kappa^{h} \in W_{\partial_{0}}^{h}$, $\vec{m}^{h}: \partial_{M} I \rightarrow \mathbb{R}^{2}$ and $\vec{Y}^{h} \in \underline{V}_{\partial_{0}}^{h}$.

Taking variations $\vec{\eta} \in \underline{V}_{\partial_{0}}^{h}$ in $\vec{Y}^{h}$, and setting $\left[\frac{\delta}{\delta \vec{Y}^{h}} \mathcal{L}^{h}\right](\vec{\eta})=0$ we obtain (4.10), similarly to (3.11). Taking variations $\chi \in W_{\partial_{0}}^{h}$ in $\kappa^{h}$ and setting $\left[\frac{\delta}{\delta \kappa^{h}} \mathcal{L}^{h}\right](\chi)=0$ we obtain

$$
2 \pi\left(\vec{X}^{h} \cdot \vec{e}_{1}\left(\alpha\left[\mathfrak{K}^{h}\left(\vec{X}^{h}, \kappa^{h}\right)-\bar{\varkappa}\right]+\beta \mathcal{A}^{h}\right), \chi\left|\vec{X}_{\rho}^{h}\right|\right)^{h}-\left(\vec{Y}^{h}, \chi \vec{\nu}^{h}\left|\vec{X}_{\rho}^{h}\right|\right)^{h}=0 \quad \forall \chi \in W_{\partial_{0}}^{h},
$$

where we have recalled (4.11). Taking variations in $\vec{m}^{h}$, and setting them to zero, yields, similarly to (3.15), that

$$
\vec{Y}^{h}=2 \pi \alpha_{G} \vec{e}_{1} \quad \text { on } \partial_{M} I .
$$

Taking variations $\vec{\chi} \in \mathbb{X}^{h}$ in $\vec{X}^{h}$, and setting $2 \pi\left(\left(\vec{X}^{h} \cdot \vec{e}_{1}\right) \underline{\underline{Q}}^{h} \vec{X}_{t}^{h}, \vec{\chi}\left|\vec{X}_{\rho}^{h}\right|\right)^{h}=-\left[\frac{\delta}{\delta \vec{X}^{h}} \mathcal{L}^{h}\right](\vec{\chi})$ we obtain

$$
\begin{aligned}
2 \pi\left(\left(\vec{X}^{h} \cdot \vec{e}_{1}\right) \underline{\underline{Q}}^{h} \vec{X}_{t}^{h}, \vec{\chi}\left|\vec{X}_{\rho}^{h}\right|\right)^{h}= & -\pi\left(\alpha\left[\mathfrak{K}^{h}\left(\vec{X}^{h}, \kappa^{h}\right)-\bar{\varkappa}\right]^{2}+2 \lambda+2 \beta \mathcal{A}^{h} \kappa^{h},\left[\frac{\delta}{\delta \vec{X}^{h}}\left(\vec{X}^{h} \cdot \vec{e}_{1}\right)\left|\vec{X}_{\rho}^{h}\right|\right](\vec{\chi})\right)^{h} \\
& -2 \pi \alpha\left(\left[\mathfrak{K}^{h}\left(\vec{X}^{h}, \kappa^{h}\right)-\bar{\varkappa}\right],\left[\frac{\delta}{\delta \vec{X}^{h}} \mathfrak{K}^{h}\left(\vec{X}^{h}, \kappa^{h}\right)\right](\vec{\chi})\left(\vec{X}^{h} \cdot \vec{e}_{1}\right)\left|\vec{X}_{\rho}^{h}\right|\right)^{h} \\
& +\left(\kappa^{h} \vec{Y}^{h}+2 \pi \beta \mathcal{A}^{h} \vec{e}_{1},\left[\frac{\delta}{\delta \vec{X}^{h}} \vec{\nu}^{h}\left|\vec{X}_{\rho}^{h}\right|\right](\vec{\chi})\right)^{h}+\left(\vec{Y}_{\rho}^{h},\left[\frac{\delta}{\delta \vec{X}^{h}} \vec{\tau}^{h}\right](\vec{\chi})\right) \\
& -2 \pi \varsigma \sum_{p \in \partial_{2} I \cup \partial_{F} I} \vec{\chi}(p) \cdot \vec{e}_{1} \quad \forall \vec{\chi} \in \mathbb{X}^{h} .
\end{aligned}
$$

Choosing $\vec{\chi}=\vec{X}_{t}^{h}$ in (4.17) yields, on noting (3.4), the discrete analogue of (3.17)

$$
\begin{aligned}
2 \pi\left(\vec{X}^{h} \cdot \vec{e}_{1}\left|\underline{\underline{Q}}^{h} \vec{X}_{t}^{h}\right|^{2},\left|\vec{X}_{\rho}^{h}\right|\right)^{h}= & -\pi\left(\alpha\left[\mathfrak{K}^{h}\left(\vec{X}^{h}, \kappa^{h}\right)-\bar{\varkappa}\right]^{2}+2 \lambda+2 \beta \mathcal{A}^{h} \kappa^{h},\left[\left(\vec{X}^{h} \cdot \vec{e}_{1}\right)\left|\vec{X}_{\rho}^{h}\right|\right]_{t}\right)^{h} \\
& -2 \pi \alpha\left(\mathfrak{K}^{h}\left(\vec{X}^{h}, \kappa^{h}\right)-\bar{\varkappa},\left[\frac{\vec{\omega}^{h} \cdot \vec{e}_{1}}{\vec{X}^{h} \cdot \vec{e}_{1}}\right]_{t}\left(\mathfrak{Z}^{h}-2\right)\left(\vec{X}^{h} \cdot \vec{e}_{1}\right)\left|\vec{X}_{\rho}^{h}\right|\right)^{h}
\end{aligned}
$$




$$
+\left(\kappa^{h} \vec{Y}^{h}+2 \pi \beta \mathcal{A}^{h} \vec{e}_{1},\left[\vec{\nu}^{h}\left|\vec{X}_{\rho}^{h}\right|\right]_{t}\right)^{h}+\left(\vec{Y}_{\rho}^{h}, \vec{\tau}_{t}^{h}\right)-2 \pi \varsigma \sum_{p \in \partial_{2} I \cup \partial_{F} I} \vec{X}_{t}^{h}(p) \cdot \vec{e}_{1} .
$$

Differentiating (4.10) with respect to $t$, and then choosing $\vec{\eta}=\vec{Y}^{h} \in \underline{V}_{\partial_{0}}^{h}$ yields, similarly to (3.18), that

$$
\left(\kappa_{t}^{h}, \vec{Y}^{h} \cdot \vec{\nu}^{h}\left|\vec{X}_{\rho}^{h}\right|\right)^{h}+\left(\kappa^{h} \vec{Y}^{h},\left[\vec{\nu}^{h}\left|\vec{X}_{\rho}^{h}\right|\right]_{t}\right)^{h}+\left(\vec{\tau}_{t}^{h}, \vec{Y}_{\rho}^{h}\right)=\sum_{p \in \partial_{M} I}\left[\vec{m}_{t}^{h} \cdot \vec{Y}^{h}\right](p) .
$$

It follows from (4.19), (4.16) and (4.15) with $\chi=\kappa_{t}^{h} \in W_{\partial_{0}}^{h}$ that

$$
\begin{aligned}
\left(\kappa^{h} \vec{Y}^{h},\left[\vec{\nu}^{h}\left|\vec{X}_{\rho}^{h}\right|\right]_{t}\right)^{h}+\left(\vec{\tau}_{t}^{h}, \vec{Y}_{\rho}^{h}\right)= & -\left(\kappa_{t}^{h}, \vec{Y}^{h} \cdot \vec{\nu}^{h}\left|\vec{X}_{\rho}^{h}\right|\right)^{h}+2 \pi \alpha_{G} \sum_{p \in \partial_{M} I}\left[\vec{m}_{t}^{h} \cdot \vec{e}_{1}\right](p) \\
= & -2 \pi\left(\vec{X}^{h} \cdot \vec{e}_{1}\left(\alpha\left[\mathfrak{K}^{h}\left(\vec{X}^{h}, \kappa^{h}\right)-\bar{\varkappa}\right]+\beta \mathcal{A}^{h}\right), \kappa_{t}^{h}\left|\vec{X}_{\rho}^{h}\right|\right)^{h} \\
& +2 \pi \alpha_{G} \sum_{p \in \partial_{M} I}\left[\vec{m}_{t}^{h}(p) \cdot \vec{e}_{1}\right] .
\end{aligned}
$$

Combining (4.18) and (4.20) yields, on recalling (4.13), the discrete analogue of (3.20)

$$
\begin{aligned}
2 \pi\left(\vec{X}^{h} \cdot \vec{e}_{1}\left|\underline{\underline{Q}}^{h} \vec{X}_{t}^{h}\right|^{2},\left|\vec{X}_{\rho}^{h}\right|\right)^{h}= & -\pi\left(\alpha\left[\mathfrak{K}^{h}\left(\vec{X}^{h}, \kappa^{h}\right)-\bar{\varkappa}^{2}+2 \lambda+2 \beta \mathcal{A}^{h} \kappa^{h},\left[\left(\vec{X}^{h} \cdot \vec{e}_{1}\right)\left|\vec{X}_{\rho}^{h}\right|\right]_{t}\right)^{h}\right. \\
& -2 \pi \alpha\left(\mathfrak{K}^{h}\left(\vec{X}^{h}, \kappa^{h}\right)-\bar{\varkappa},\left[\frac{\vec{\omega}^{h} \cdot \vec{e}_{1}}{\vec{X}^{h} \cdot \vec{e}_{1}}\right]_{t}\left(\mathfrak{Z}^{h}-2\right)\left(\vec{X}^{h} \cdot \vec{e}_{1}\right)\left|\vec{X}_{\rho}^{h}\right|\right)^{h} \\
& -2 \pi\left(\vec{X}^{h} \cdot \vec{e}_{1}\left(\alpha\left[\mathfrak{K}^{h}\left(\vec{X}^{h}, \kappa^{h}\right)-\bar{\varkappa}\right]+\beta \mathcal{A}^{h}\right), \kappa_{t}^{h}\left|\vec{X}_{\rho}^{h}\right|\right)^{h} \\
& +2 \pi \beta \mathcal{A}^{h}\left(\vec{e}_{1},\left[\vec{\nu}^{h}\left|\vec{X}_{\rho}^{h}\right|\right]_{t}\right)^{h} \\
& +2 \pi \alpha_{G} \sum_{p \in \partial_{M} I}\left[\vec{m}_{t}^{h}(p) \cdot \vec{e}_{1}\right]-2 \pi \varsigma \sum_{p \in \partial_{2} I \cup \partial_{F} I} \vec{X}_{t}^{h}(p) \cdot \vec{e}_{1} \\
= & -\frac{\mathrm{d}}{\mathrm{d} t} \widehat{E}^{h}(t) .
\end{aligned}
$$

We now return to (4.17), which, on recalling (4.8), (4.11), (4.12) and (4.9), can be rewritten as

$$
\begin{aligned}
& 2 \pi\left(\left(\vec{X}^{h} \cdot \vec{e}_{1}\right) \underline{\underline{Q}}^{h} \vec{X}_{t}^{h}, \vec{\chi}\left|\vec{X}_{\rho}^{h}\right|\right)^{h}=-\pi\left(\alpha\left[\mathfrak{K}^{h}\left(\vec{X}^{h}, \kappa^{h}\right)-\bar{\varkappa}\right]^{2}+2 \lambda+2 \beta \mathcal{A}^{h} \kappa^{h}, \vec{\chi} \cdot \vec{e}_{1}\left|\vec{X}_{\rho}^{h}\right|+\left(\vec{X}^{h} \cdot \vec{e}_{1}\right) \vec{\tau}^{h} \cdot \vec{\chi}_{\rho}\right)^{h} \\
& +2 \pi \alpha\left(\left[\mathfrak{K}^{h}\left(\vec{X}^{h}, \kappa^{h}\right)-\bar{\varkappa}\right]\left(\mathfrak{Z}^{h}-2\right), \frac{\vec{\omega}^{h} \cdot \vec{e}_{1}}{\vec{X}^{h} \cdot \vec{e}_{1}} \vec{\chi} \cdot \vec{e}_{1}\left|\vec{X}_{\rho}^{h}\right|\right)^{h} \\
& +2 \pi \alpha\left(\left[\mathfrak{K}^{h}\left(\vec{X}^{h}, \kappa^{h}\right)-\bar{\varkappa}\right]\left(\mathfrak{Z}^{h}-2\right) \vec{e}_{1},\left(\vec{\nu}^{h} \cdot \vec{\chi}_{\rho}\right) \vec{\tau}^{h}+\left(\vec{\tau}^{h} \cdot \vec{\chi}_{\rho}\right)\left(\vec{\omega}^{h}-\vec{\nu}^{h}\right)\right)^{h} \\
& -\left(\kappa^{h} \vec{Y}^{h}+2 \pi \beta \mathcal{A}^{h} \vec{e}_{1}, \vec{\chi}_{\rho}^{\perp}\right)^{h}+\left(\vec{Y}_{\rho}^{h} \cdot \vec{\nu}^{h}, \vec{\chi}_{\rho} \cdot \vec{\nu}^{h}\left|\vec{X}_{\rho}^{h}\right|^{-1}\right) \\
& -2 \pi \varsigma \sum_{p \in \partial_{2} I \cup \partial_{F} I} \vec{\chi}(p) \cdot \vec{e}_{1} \quad \forall \vec{\chi} \in \mathbb{X}^{h} \text {. }
\end{aligned}
$$

Combining (4.22), (4.15), (4.10), (4.16) and (4.14), our semidiscrete approximation based on $\kappa^{h}$ is given, on noting (3.1a) and (4.4), as follows. 
$\left(\mathcal{P}^{h}\right)^{h}$ Let $\vec{X}^{h}(\cdot, 0) \in \underline{V}_{\partial_{0}}^{h}$ and $\alpha \in \mathbb{R}_{>0}, \bar{\varkappa}, M_{0}, \alpha_{G}, \lambda, \varsigma \in \mathbb{R}, \beta \in \mathbb{R}_{\geq 0}, \vec{\zeta}: \partial_{C} I \rightarrow \mathbb{S}^{1}$ be given. Then, for $t \in(0, T]$ find $\vec{X}^{h}(\cdot, t) \in \underline{V}_{\partial_{0}}^{h}$, with $\vec{X}_{t}^{h}(\cdot, t) \in \mathbb{X}^{h}, \kappa^{h}(\cdot, t) \in W_{\partial_{0}}^{h}, \vec{Y}^{h}(\cdot, t) \in \mathbb{Y}^{h}\left(2 \pi \alpha_{G} \vec{e}_{1}\right)$ and $\vec{m}^{h}(\cdot, t): \partial_{M} I \rightarrow \mathbb{R}^{2}$ such that

$$
\begin{aligned}
& 2 \pi\left(\left(\vec{X}^{h} \cdot \vec{e}_{1}\right) \underline{\underline{Q}}^{h} \vec{X}_{t}^{h}, \vec{\chi}\left|\vec{X}_{\rho}^{h}\right|\right)^{h}-\left(\vec{Y}_{\rho}^{h} \cdot \vec{\nu}^{h}, \vec{\chi}_{\rho} \cdot \vec{\nu}^{h}\left|\vec{X}_{\rho}^{h}\right|^{-1}\right) \\
& =-\pi\left(\alpha\left[\mathfrak{K}^{h}\left(\vec{X}^{h}, \kappa^{h}\right)-\bar{\varkappa}\right]^{2}+2 \lambda+2 \beta \mathcal{A}^{h} \kappa^{h}, \vec{\chi} \cdot \vec{e}_{1}\left|\vec{X}_{\rho}^{h}\right|+\left(\vec{X}^{h} \cdot \vec{e}_{1}\right) \vec{\tau}^{h} \cdot \vec{\chi}_{\rho}\right)^{h} \\
& +2 \pi \alpha\left(\left[\mathfrak{K}^{h}\left(\vec{X}^{h}, \kappa^{h}\right)-\bar{\varkappa}\right]\left(\mathfrak{Z}^{h}-2\right) \frac{\vec{\omega}^{h} \cdot \vec{e}_{1}}{\vec{X}^{h} \cdot \vec{e}_{1}} \vec{\chi} \cdot \vec{e}_{1}\left|\vec{X}_{\rho}^{h}\right|\right)^{h} \\
& +2 \pi \alpha\left(\left[\mathfrak{K}^{h}\left(\vec{X}^{h}, \kappa^{h}\right)-\bar{\varkappa}\right]\left(\mathfrak{Z}^{h}-2\right) \vec{e}_{1},\left(\vec{\nu}^{h} \cdot \vec{\chi}_{\rho}\right) \vec{\tau}^{h}+\left(\vec{\tau}^{h} \cdot \vec{\chi}_{\rho}\right)\left(\vec{\omega}^{h}-\vec{\nu}^{h}\right)\right)^{h} \\
& +\left(\kappa^{h}\left(\vec{Y}^{h}\right)^{\perp}-2 \pi \beta \mathcal{A}^{h} \vec{e}_{2}, \vec{\chi}_{\rho}\right)^{h}-2 \pi \varsigma \sum_{p \in \partial_{2} I \cup \partial_{F} I} \vec{\chi}(p) \cdot \vec{e}_{1} \quad \forall \vec{\chi} \in \mathbb{X}^{h}, \\
& 2 \pi\left(\vec{X}^{h} \cdot \vec{e}_{1}\left(\alpha\left[\mathfrak{K}^{h}\left(\vec{X}^{h}, \kappa^{h}\right)-\bar{\varkappa}\right]+\beta \mathcal{A}^{h}\right), \chi\left|\vec{X}_{\rho}^{h}\right|\right)^{h}-\left(\vec{Y}^{h}, \chi \vec{\nu}^{h}\left|\vec{X}_{\rho}^{h}\right|\right)^{h}=0 \quad \forall \chi \in W_{\partial_{0}}^{h}, \\
& \left(\kappa^{h} \vec{\nu}^{h}, \vec{\eta}\left|\vec{X}_{\rho}^{h}\right|\right)^{h}+\left(\vec{X}_{\rho}^{h}, \vec{\eta}_{\rho}\left|\vec{X}_{\rho}^{h}\right|^{-1}\right)=\sum_{p \in \partial_{C} I}[\vec{\zeta} \cdot \vec{\eta}](p)+\sum_{p \in \partial_{M} I}\left[\vec{m}^{h} \cdot \vec{\eta}\right](p) \quad \forall \vec{\eta} \in \underline{V}_{\partial_{0}}^{h},
\end{aligned}
$$

where $\mathcal{A}^{h}(t)$ is given by (4.14).

Theorem 4.4. Let Assumption 4.2 be satisfied and let $\left(\vec{X}^{h}(t), \kappa^{h}(t), \vec{Y}^{h}(t), \vec{m}^{h}(t)\right)_{t \in(0, T]}$ be a solution to (4.23). Then the solution satisfies the stability bound

$$
\frac{\mathrm{d}}{\mathrm{d} t} \widehat{E}^{h}(t)+2 \pi\left(\vec{X}^{h} \cdot \vec{e}_{1}\left|\underline{\underline{Q^{h}}} \vec{X}_{t}^{h}\right|^{2},\left|\vec{X}_{\rho}^{h}\right|\right)^{h}=0 .
$$

Proof. The desired result follows as (4.23) is just a rewrite of (4.17), (4.15), (4.10) and (4.14), and then noting (4.18)-(4.21).

Remark 4.5. We note that on choosing $\vec{\eta}=\chi_{j}\left[\vec{\omega}^{h}\left(q_{j}\right)\right]^{\perp}$, for $j \in\left\{1, \ldots, j_{1}\right\}$ so $\vec{\eta} \in \underline{V}_{\partial_{0}}^{h}$, in (4.23c) we obtain that

$$
\left|\vec{X}^{h}\left(q_{j}\right)-\vec{X}^{h}\left(q_{j-1}\right)\right|=\left|\vec{X}^{h}\left(q_{j+1}\right)-\vec{X}^{h}\left(q_{j}\right)\right| \text { or } \vec{X}^{h}\left(q_{j}\right)-\vec{X}^{h}\left(q_{j-1}\right) \| \vec{X}^{h}\left(q_{j+1}\right)-\vec{X}^{h}\left(q_{j}\right)
$$

for $j=1, \ldots, j_{1}$. See Remark 2.4 of [1] for details. Hence the curve $\Gamma^{h}(t)$ will be equidistributed where-ever two neighbouring elements are not parallel. This aspect of the solution for the semidiscrete problem (4.23) means that it can be viewed as a highly nonlinear and degenerate system of differential-algebraic equations, see also Remark 81 of [11] for a related discussion. In particular, at present we are unable to prove the existence of solutions to (4.23). In addition, an error analysis for our semidiscrete approximations also appears to be out of reach.

Remark 4.6. We now revisit the discussion in Remark 4.3. Assuming that e.g. $0=q_{0} \in \partial_{0} I$, then choosing $\vec{\eta}=\chi_{0} \vec{e}_{2}$ in (4.23c) yields that $\kappa^{h}\left(q_{0}\right) \vec{\nu}^{h}\left(q_{0}\right) \cdot \vec{e}_{2}=2 \frac{\left(\vec{X}^{h}\left(q_{1}\right)-\vec{X}^{h}\left(q_{0}\right)\right) \cdot \vec{e}_{2}}{\left|\vec{X}^{h}\left(q_{1}\right)-\vec{X}^{h}\left(q_{0}\right)\right|^{2}}$, and so

$$
\vec{\tau}^{h}\left(q_{0}\right) \cdot \vec{e}_{2}=\frac{1}{2}\left|\vec{X}^{h}\left(q_{1}\right)-\vec{X}^{h}\left(q_{0}\right)\right| \kappa^{h}\left(q_{0}\right) \vec{\nu}^{h}\left(q_{0}\right) \cdot \vec{e}_{2},
$$

where we have noted (4.4). Clearly, (4.24) is a discrete approximation of (2.11), which stipulates a $90^{\circ}$ degree contact angle between $\Gamma(t)$ and the $x_{2}$-axis.

For the scheme (4.23) we fix $\kappa^{h} \in W_{\partial_{0}}^{h}$, and so the right hand side in (4.24) is zero. For a more general scheme, where we allow $\kappa^{h} \in V^{h}$ and let $\chi \in V^{h}$ in (4.23b), the right hand side in (4.24) is still of order $O\left(J^{-1}\right)$ on 
assuming that $\kappa^{h}\left(q_{0}\right)$ is bounded. However, we observe that $\kappa^{h}\left(q_{0}\right)$, if $0 \in \partial_{0} I$, only appears in (4.23c) for this more general scheme. Hence there is no reason to assume that $\kappa^{h}\left(q_{0}\right)$ should remain bounded. In fact, $\kappa^{h}\left(q_{0}\right)$ simply acts as a register for the value $\frac{\vec{\tau}^{h}\left(q_{0}\right) \cdot \vec{e}_{2}}{\vec{\nu}^{h}\left(q_{0}\right) \cdot \vec{e}_{2}} \frac{2 J}{\mathcal{H}^{1}\left(\Gamma^{h}(t)\right)}$. Moreover, a fully discrete variant of the discussed more general version of (4.23) leads to incorrect contact angles at the $x_{2}$-axis and to the numerical breakdown of the scheme. This is the main reason why we consider the scheme (4.23) as it is.

\subsubsection{Conserved flows}

We rewrite (4.23a) as

$$
2 \pi\left(\left(\vec{X}^{h} \cdot \vec{e}_{1}\right) \underline{\underline{Q}}^{h} \vec{X}_{t}^{h}, \vec{\chi}\left|\vec{X}_{\rho}^{h}\right|\right)^{h}-\left(\vec{Y}_{\rho}^{h} \cdot \vec{\nu}^{h}, \vec{\chi}_{\rho} \cdot \vec{\nu}^{h}\left|\vec{X}_{\rho}^{h}\right|^{-1}\right)=\left(\vec{f}^{h}, \vec{\chi}\left|\vec{X}_{\rho}^{h}\right|\right)^{h} \quad \forall \vec{\chi} \in \mathbb{X}^{h} .
$$

Then the natural generalization of $\left(\mathcal{P}^{h}\right)^{h},(4.23)$, that approximates the weak formulation (3.26), (3.22b), (3.22c) and (3.31) is given by (4.23), with (4.23a) replaced by

$$
\begin{aligned}
2 \pi\left(\left(\vec{X}^{h} \cdot \vec{e}_{1}\right)\right. & \left.\underline{\underline{Q}}^{h} \vec{X}_{t}^{h}, \vec{\chi}\left|\vec{X}_{\rho}^{h}\right|\right)^{h}-\left(\vec{Y}_{\rho}^{h} \cdot \vec{\nu}^{h}, \vec{\chi}_{\rho} \cdot \vec{\nu}^{h}\left|\vec{X}_{\rho}^{h}\right|^{-1}\right)=\left(\vec{f}^{h}, \vec{\chi}\left|\vec{X}_{\rho}^{h}\right|\right)^{h} \\
& -2 \pi \lambda_{A}^{h}\left[\left(\vec{e}_{1}, \vec{\chi}\left|\vec{X}_{\rho}^{h}\right|\right)+\left(\left(\vec{X}^{h} \cdot \vec{e}_{1}\right) \vec{\tau}^{h}, \vec{\chi}_{\rho}\right)\right]-2 \pi \lambda_{V}^{h}\left(\left(\vec{X}^{h} \cdot \vec{e}_{1}\right) \vec{\nu}^{h}, \vec{\chi}\left|\vec{X}_{\rho}^{h}\right|\right) \quad \forall \vec{\chi} \in \mathbb{X}^{h},
\end{aligned}
$$

where $\left(\lambda_{A}^{h}(t), \lambda_{V}^{h}(t)\right)^{T} \in \mathbb{R}^{2}$ are such that

$$
A\left(\vec{X}^{h}(t)\right)=A\left(\vec{X}^{h}(0)\right) \text { and } V\left(\vec{X}^{h}(t)\right)=V\left(\vec{X}^{h}(0)\right) .
$$

Here, on recalling (3.29) and (3.30), we note that $A\left(\vec{X}^{h}(t)\right)$ denotes the surface area of $\mathcal{S}^{h}(t)$, where, similarly to $(2.1)$, we set

$$
\mathcal{S}^{h}(t)=\bigcup_{\rho \in \bar{I}} \Pi_{2}^{3}\left(\vec{X}^{h}(\rho, t)\right)
$$

Moreover, $V\left(\vec{X}^{h}(t)\right)$ is the volume of the domain $\Omega^{h}(t)$ with $\partial \Omega^{h}(t)=\mathcal{S}^{h}(t)$ in the case that $\mathcal{S}^{h}(t)$ has no boundary. We remark that

$$
A\left(\vec{Z}^{h}\right)=2 \pi\left(\vec{Z}^{h} \cdot \vec{e}_{1},\left|\vec{Z}_{\rho}^{h}\right|\right) \quad \vec{Z}^{h} \in \underline{V}_{\partial_{0}}^{h}
$$

and

$$
V\left(\vec{Z}^{h}\right)=-\pi\left(\left(\vec{Z}^{h} \cdot \vec{e}_{1}\right)^{2},\left[\vec{Z}_{\rho}^{h}\right]^{\perp} \cdot \vec{e}_{1}\right) \quad \vec{Z}^{h} \in \underline{V}_{\partial_{0}}^{h}
$$

recall (3.29), (3.30) and (4.4). Moreover, we recall from (3.7) and (3.11) of [7] that, similarly to (2.38) and (2.39), it holds that

and

$$
\frac{\mathrm{d}}{\mathrm{d} t} A\left(\vec{X}^{h}(t)\right)=2 \pi\left[\left(\vec{e}_{1}, \vec{X}_{t}^{h}\left|\vec{X}_{\rho}^{h}\right|\right)+\left(\left(\vec{X}^{h} \cdot \vec{e}_{1}\right) \vec{\tau}^{h},\left(\vec{X}_{t}^{h}\right)_{\rho}\right)\right]
$$

$$
\frac{\mathrm{d}}{\mathrm{d} t} V\left(\vec{X}^{h}(t)\right)=2 \pi\left(\left(\vec{X}^{h} \cdot \vec{e}_{1}\right) \vec{\nu}^{h}, \vec{X}_{t}^{h}\left|\vec{X}_{\rho}^{h}\right|\right)
$$

Theorem 4.7. Let Assumption 4.2 be satisfied and let $\left(\vec{X}^{h}(t), \kappa^{h}(t), \vec{Y}^{h}(t), \vec{m}^{h}(t), \lambda_{A}^{h}(t), \lambda_{V}^{h}(t)\right)_{t \in(0, T]}$ be a solution to (4.25), (4.23b), (4.23c), (4.26). Then the solution satisfies the stability bound

$$
\frac{\mathrm{d}}{\mathrm{d} t} \widehat{E}^{h}(t)+2 \pi\left(\vec{X}^{h} \cdot \vec{e}_{1}\left|\underline{\underline{Q}}^{h} \vec{X}_{t}^{h}\right|^{2},\left|\vec{X}_{\rho}^{h}\right|\right)^{h}=0 .
$$

Proof. Differentiating the two equations in (4.26) with respect to $t$, noting (4.27), (4.28), and choosing $\vec{\chi}=\vec{X}_{t}^{h}$ in $(4.25)$ yields

$$
2 \pi\left(\vec{X}^{h} \cdot \vec{e}_{1}\left|\underline{\underline{Q^{h}}} \vec{X}_{t}^{h}\right|^{2},\left|\vec{X}_{\rho}^{h}\right|\right)^{h}-\left(\vec{Y}_{\rho}^{h} \cdot \vec{\nu}^{h},\left(\vec{X}_{t}^{h}\right)_{\rho} \cdot \vec{\nu}^{h}\left|\vec{X}_{\rho}^{h}\right|^{-1}\right)=\left(\vec{f}^{h}, \vec{X}_{t}^{h}\left|\vec{X}_{\rho}^{h}\right|\right)^{h},
$$

which is equivalent to (4.18). Hence the stability result follows as in the proof of Theorem 4.4. 


\subsection{Based on $\kappa_{\mathcal{S}}^{h}$}

The approach in Section 4.1 required the introduction of the auxiliary finite element function (4.11) in order to be able to obtain a well-defined Lagrangian. An alternative is to define the Lagrangian in terms of $\kappa_{\mathcal{S}}^{h}$, a direct discrete analogue of $\varkappa_{\mathcal{S}}$. Then it is possible to repeat the proof of (3.42) on the discrete level.

As the discrete analogue of (3.9), we let $\vec{X}^{h} \in \underline{V}_{\partial_{0}}^{h}, \kappa_{\mathcal{S}}^{h} \in W_{\left(\partial_{0}\right)}^{h}$ and $\vec{m}^{h}: \partial_{M} I \rightarrow \mathbb{R}^{2}$ be such that

$$
\begin{aligned}
& \left(\vec{X}^{h} \cdot \vec{e}_{1} \kappa_{\mathcal{S}}^{h} \vec{\nu}^{h}, \vec{\eta}\left|\vec{X}_{\rho}^{h}\right|\right)^{(h)}+\left(\vec{e}_{1}, \vec{\eta}\left|\vec{X}_{\rho}^{h}\right|\right)+\left(\left(\vec{X}^{h} \cdot \vec{e}_{1}\right) \vec{\tau}^{h}, \vec{\eta}_{\rho}\right) \\
& \quad=\sum_{p \in \partial_{C} I}\left[\left(\vec{X}^{h} \cdot \vec{e}_{1}\right) \vec{\zeta} \cdot \vec{\eta}\right](p)+\sum_{p \in \partial_{M} I}\left[\left(\vec{X}^{h} \cdot \vec{e}_{1}\right) \vec{m}^{h} \cdot \vec{\eta}\right](p) \quad \forall \vec{\eta} \in \underline{V}_{\partial_{0}}^{h} .
\end{aligned}
$$

Here, and throughout, we use the notation ${ }^{(h)}$ to denote an expression with or without the superscript $h$, and simultaneously for the notation with subscripts $\cdot\left(\partial_{0}\right)$. i.e. we consider two separate situations: either mass lumping is used on the first integral, and then we let $\kappa_{\mathcal{S}}^{h} \in W_{\partial_{0}}^{h}$, or true integration is employed and we let $\kappa_{\mathcal{S}}^{h} \in W^{h}$. We define the discrete analogue of $(2.36)$

$$
\widehat{E}_{\mathcal{S}}^{h}(t)=\pi\left(\alpha\left[\kappa_{\mathcal{S}}^{h}-\bar{\varkappa}\right]^{2}+2 \lambda, \vec{X}^{h} \cdot \vec{e}_{1}\left|\vec{X}_{\rho}^{h}\right|\right)^{(h)}+\frac{\beta}{2}\left(\mathcal{A}_{\mathcal{S}}^{h}(t)\right)^{2}-2 \pi \alpha_{G} \sum_{p \in \partial_{M} I} \vec{m}^{h}(p) \cdot \vec{e}_{1}+2 \pi \varsigma \sum_{p \in \partial_{2} I \cup \partial_{F} I} \vec{X}^{h}(p) \cdot \vec{e}_{1},
$$

where

$$
\mathcal{A}_{\mathcal{S}}^{h}(t)=2 \pi\left(\vec{X}^{h} \cdot \vec{e}_{1} \kappa_{\mathcal{S}}^{h},\left|\vec{X}_{\rho}^{h}\right|\right)^{(h)}-M_{0} .
$$

Similarly to (3.32), we define the discrete Lagrangian

$$
\begin{aligned}
\mathcal{L}_{\mathcal{S}}^{h}\left(\vec{X}^{h}, \kappa_{\mathcal{S}}^{h}, \vec{m}^{h}, \vec{Y}_{\mathcal{S}}^{h}\right)= & \pi\left(\alpha\left[\kappa_{\mathcal{S}}^{h}-\bar{\varkappa}\right]^{2}+2 \lambda, \vec{X}^{h} \cdot \vec{e}_{1}\left|\vec{X}_{\rho}^{h}\right|\right)^{(h)}+\frac{\beta}{2}\left[2 \pi\left(\vec{X}^{h} \cdot \vec{e}_{1} \kappa_{\mathcal{S}}^{h},\left|\vec{X}_{\rho}^{h}\right|\right)^{(h)}-M_{0}\right]^{2} \\
& -\left(\vec{X}^{h} \cdot \vec{e}_{1} \kappa_{\mathcal{S}}^{h} \vec{\nu}^{h}+\vec{e}_{1}, \vec{Y}_{\mathcal{S}}^{h}\left|\vec{X}_{\rho}^{h}\right|\right)^{(h)}-\left(\left(\vec{X}^{h} \cdot \vec{e}_{1}\right) \vec{\tau}^{h},\left(\vec{Y}_{\mathcal{S}}^{h}\right)_{\rho}\right)+2 \pi \varsigma \sum_{p \in \partial_{2} I \cup \partial_{F} I} \vec{X}^{h}(p) \\
& \cdot \vec{e}_{1}+\sum_{p \in \partial_{C} I}\left[\left(\vec{X}^{h} \cdot \vec{e}_{1}\right) \vec{\zeta} \cdot \vec{Y}_{\mathcal{S}}^{h}\right](p)+\sum_{p \in \partial_{M} I}\left[\vec{m}^{h} \cdot\left(\left(\vec{X}^{h} \cdot \vec{e}_{1}\right) \vec{Y}_{\mathcal{S}}^{h}-2 \pi \alpha_{G} \vec{e}_{1}\right)\right](p),
\end{aligned}
$$

for $\vec{X}^{h} \in \underline{V}_{\partial_{0}}^{h}, \kappa_{\mathcal{S}}^{h} \in W_{\left(\partial_{0}\right)}^{h}, \vec{m}^{h}: \partial_{M} I \rightarrow \mathbb{R}^{2}$ and $\vec{Y}_{\mathcal{S}}^{h} \in \underline{V}_{\partial_{0}}^{h}$. Here we observe that in the case of numerical integration, we fix $\kappa_{\mathcal{S}}^{h}$ to be zero on the boundary $\partial_{0} I$, as the Lagrangian (4.32) does not depend on these boundary values at all.

Taking variations $\vec{\eta} \in \underline{V}_{\partial_{0}}^{h}$ in $\vec{Y}_{\mathcal{S}}^{h}$, and setting $\left[\frac{\delta}{\delta \vec{Y}_{\mathcal{S}}^{h}} \mathcal{L}_{\mathcal{S}}^{h}\right](\vec{\eta})=0$ we obtain (4.29), similarly to (3.33). Taking variations $\chi \in W_{\left(\partial_{0}\right)}^{h}$ in $\kappa_{\mathcal{S}}^{h}$ and setting $\left[\frac{\delta}{\delta \kappa_{\mathcal{S}}^{h}} \mathcal{L}_{\mathcal{S}}^{h}\right](\chi)=0$ we obtain, similarly to (3.34),

$$
2 \pi\left(\alpha\left(\kappa_{\mathcal{S}}^{h}-\bar{\varkappa}\right)+\beta \mathcal{A}_{\mathcal{S}}^{h}, \vec{X}^{h} \cdot \vec{e}_{1} \chi\left|\vec{X}_{\rho}^{h}\right|\right)^{(h)}-\left(\left(\vec{X}^{h} \cdot \vec{e}_{1}\right) \vec{Y}_{\mathcal{S}}^{h}, \chi \vec{\nu}^{h}\left|\vec{X}_{\rho}^{h}\right|\right)^{(h)}=0 \quad \forall \chi \in W_{\left(\partial_{0}\right)}^{h} .
$$

Taking variations in $\vec{m}^{h}$, and setting them to zero, yields, similarly to (3.37), that

$$
\left(\vec{X}^{h} \cdot \vec{e}_{1}\right) \vec{Y}_{\mathcal{S}}^{h}=2 \pi \alpha_{G} \vec{e}_{1} \quad \text { on } \partial_{M} I
$$

Taking variations $\vec{\chi} \in \mathbb{X}^{h}$ in $\vec{X}^{h}$ and setting $2 \pi\left(\left(\vec{X}^{h} \cdot \vec{e}_{1}\right) \underline{\underline{Q}}^{h} \vec{X}_{t}^{h}, \vec{\chi}\left|\vec{X}_{\rho}^{h}\right|\right)^{h}=-\left[\frac{\delta}{\delta \vec{X}^{h}} \mathcal{L}_{\mathcal{S}}^{h}\right](\vec{\chi})$ we obtain, similarly to (3.38), that

$$
2 \pi\left(\left(\vec{X}^{h} \cdot \vec{e}_{1}\right) \underline{\underline{Q}}^{h} \vec{X}_{t}^{h}, \vec{\chi}\left|\vec{X}_{\rho}^{h}\right|\right)^{h}=-\pi\left(\alpha\left[\kappa_{\mathcal{S}}^{h}-\bar{\varkappa}\right]^{2}+2 \lambda+2 \beta \mathcal{A}_{\mathcal{S}}^{h} \kappa_{\mathcal{S}}^{h},\left[\frac{\delta}{\delta \vec{X}^{h}}\left(\vec{X}^{h} \cdot \vec{e}_{1}\right)\left|\vec{X}_{\rho}^{h}\right|\right](\vec{\chi})\right)^{(h)}
$$




$$
\begin{aligned}
& +\left(\kappa_{\mathcal{S}}^{h} \vec{Y}_{\mathcal{S}}^{h},\left[\frac{\delta}{\delta \vec{X}^{h}}\left(\vec{X}^{h} \cdot \vec{e}_{1}\right) \vec{\nu}^{h}\left|\vec{X}_{\rho}^{h}\right|\right](\vec{\chi})\right)^{(h)}+\left(\vec{e}_{1} \cdot \vec{Y}_{\mathcal{S}}^{h},\left[\frac{\delta}{\delta \vec{X}^{h}}\left|\vec{X}_{\rho}^{h}\right|\right](\vec{\chi})\right) \\
& \times\left(\left(\vec{Y}_{\mathcal{S}}^{h}\right)_{\rho},\left[\frac{\delta}{\delta \vec{X}^{h}}\left(\vec{X}^{h} \cdot \vec{e}_{1}\right) \vec{\tau}^{h}\right](\vec{\chi})\right) \\
& -\sum_{p \in \partial_{2} I \cup \partial_{F} I}\left[\left[2 \pi \varsigma+\vec{m}^{h} \cdot \vec{Y}_{\mathcal{S}}^{h}\right]\left(\vec{\chi} \cdot \vec{e}_{1}\right)\right](p) \quad \forall \vec{\chi} \in \mathbb{X}^{h} .
\end{aligned}
$$

Choosing $\vec{\chi}=\vec{X}_{t}^{h} \in \mathbb{X}^{h}$ in (4.35), on noting (3.4), yields the discrete analogue of (3.39)

$$
\begin{aligned}
2 \pi\left(\vec{X}^{h} \cdot \vec{e}_{1}\left|\underline{\underline{Q}}^{h} \vec{X}_{t}^{h}\right|^{2},\left|\vec{X}_{\rho}^{h}\right|\right)^{h}= & -\pi\left(\alpha\left[\kappa_{\mathcal{S}}^{h}-\vec{\varkappa}^{2}+2 \lambda+2 \beta \mathcal{A}_{\mathcal{S}}^{h} \kappa_{\mathcal{S}}^{h},\left[\left(\vec{X}^{h} \cdot \vec{e}_{1}\right)\left|\vec{X}_{\rho}^{h}\right|\right]_{t}\right)^{(h)}\right. \\
& +\left(\kappa_{\mathcal{S}}^{h} \vec{Y}_{\mathcal{S}}^{h},\left[\left(\vec{X}^{h} \cdot \vec{e}_{1}\right) \vec{\nu}^{h}\left|\vec{X}_{\rho}^{h}\right|\right]_{t}\right)^{(h)}+\left(\vec{e}_{1} \cdot \vec{Y}_{\mathcal{S}}^{h},\left(\left|\vec{X}_{\rho}^{h}\right|\right)_{t}\right) \\
& +\left(\left(\vec{Y}_{\mathcal{S}}^{h}\right)_{\rho},\left[\left(\vec{X}^{h} \cdot \vec{e}_{1}\right) \vec{\tau}^{h}\right]_{t}\right)-\sum_{p \in \partial_{2} I \cup \partial_{F} I}\left[\left[2 \pi \varsigma+\vec{m}^{h} \cdot \vec{Y}_{\mathcal{S}}^{h}\right]\left(\vec{X}_{t}^{h} \cdot \vec{e}_{1}\right)\right](p) .
\end{aligned}
$$

Differentiating (4.29) with respect to $t$, and then choosing $\vec{\eta}=\vec{Y}_{\mathcal{S}}^{h} \in \underline{V}_{\partial_{0}}^{h}$, we obtain, on recalling that $\vec{X}_{t}^{h} \in \mathbb{X}^{h}$,

$$
\begin{gathered}
\left(\left(\kappa_{\mathcal{S}}^{h}\right)_{t} \vec{Y}_{\mathcal{S}}^{h},\left(\vec{X}^{h} \cdot \vec{e}_{1}\right) \vec{\nu}^{h}\left|\vec{X}_{\rho}^{h}\right|\right)^{(h)}+\left(\kappa_{\mathcal{S}}^{h} \vec{Y}_{\mathcal{S}}^{h},\left[\left(\vec{X}^{h} \cdot \vec{e}_{1}\right) \vec{\nu}^{h}\left|\vec{X}_{\rho}^{h}\right|\right]_{t}\right)^{(h)}+\left(\vec{e}_{1} \cdot \vec{Y}_{\mathcal{S}}^{h},\left[\left|\vec{X}_{\rho}^{h}\right|\right]_{t}\right) \\
+\left(\left(\vec{Y}_{\mathcal{S}}^{h}\right)_{\rho},\left[\left(\vec{X}^{h} \cdot \vec{e}_{1}\right) \vec{\tau}^{h}\right]_{t}\right)=\sum_{p \in \partial_{M} I}\left[\left(\vec{X}_{t}^{h} \cdot \vec{e}_{1}\right) \vec{m}^{h} \cdot \vec{Y}_{\mathcal{S}}^{h}+\left(\vec{X}^{h} \cdot \vec{e}_{1}\right)\left(\vec{m}^{h}\right)_{t} \cdot \vec{Y}_{\mathcal{S}}^{h}\right](p) .
\end{gathered}
$$

It follows from (4.37), (4.34) and (4.33) with $\chi=\left(\kappa_{\mathcal{S}}^{h}\right)_{t} \in W_{\left(\partial_{0}\right)}^{h}$ that

$$
\begin{aligned}
& \left(\kappa_{\mathcal{S}}^{h} \vec{Y}_{\mathcal{S}}^{h},\left[\left(\vec{X}^{h} \cdot \vec{e}_{1}\right) \vec{\nu}^{h}\left|\vec{X}_{\rho}^{h}\right|\right]_{t}\right)^{(h)}+\left(\vec{e}_{1} \cdot \vec{Y}_{\mathcal{S}}^{h},\left[\left|\vec{X}_{\rho}^{h}\right|\right]_{t}\right)+\left(\left(\vec{Y}_{\mathcal{S}}^{h}\right)_{\rho},\left[\left(\vec{X}^{h} \cdot \vec{e}_{1}\right) \vec{\tau}^{h}\right]_{t}\right) \\
& \quad=-\left(\left(\kappa_{\mathcal{S}}^{h}\right)_{t},\left(\vec{X}^{h} \cdot \vec{e}_{1}\right) \vec{Y}_{\mathcal{S}}^{h} \cdot \vec{\nu}^{h}\left|\vec{X}_{\rho}^{h}\right|\right)^{(h)}+\sum_{p \in \partial_{M} I}\left[\left(\vec{X}_{t}^{h} \cdot \vec{e}_{1}\right) \vec{m}^{h} \cdot \vec{Y}_{\mathcal{S}}^{h}+2 \pi \alpha_{G} \vec{m}_{t}^{h} \cdot \vec{e}_{1}\right](p) \\
& \quad=-2 \pi\left(\alpha\left(\kappa_{\mathcal{S}}^{h}-\bar{\varkappa}\right)+\beta \mathcal{A}_{\mathcal{S}}^{h}, \vec{X}^{h} \cdot \vec{e}_{1}\left(\kappa_{\mathcal{S}}^{h}\right)_{t}\left|\vec{X}_{\rho}^{h}\right|\right)^{(h)}+\sum_{p \in \partial_{M} I}\left[\left(\vec{X}_{t}^{h} \cdot \vec{e}_{1}\right) \vec{m}^{h} \cdot \vec{Y}_{\mathcal{S}}^{h}+2 \pi \alpha_{G} \vec{m}_{t}^{h} \cdot \vec{e}_{1}\right](p) .
\end{aligned}
$$

Combining (4.36) and (4.38) yields the discrete analogue of (3.42)

$$
\begin{aligned}
& 2 \pi\left(\vec{X}^{h} \cdot \vec{e}_{1}\left|\underline{\underline{Q}}^{h} \vec{X}_{t}^{h}\right|^{2},\left|\vec{X}_{\rho}^{h}\right|\right)^{h}=-\pi\left(\alpha\left[\kappa_{\mathcal{S}}^{h}-\bar{\varkappa}\right]^{2}+2 \lambda+2 \beta \mathcal{A}_{\mathcal{S}}^{h} \kappa_{\mathcal{S}}^{h},\left[\vec{X}^{h} \cdot \vec{e}_{1}\left|\vec{X}_{\rho}^{h}\right|\right]_{t}\right)^{(h)} \\
& \quad-2 \pi\left(\alpha\left(\kappa_{\mathcal{S}}^{h}-\bar{\varkappa}\right)+\beta \mathcal{A}_{\mathcal{S}}^{h}, \vec{X}^{h} \cdot \vec{e}_{1}\left(\kappa_{\mathcal{S}}^{h}\right)_{t}\left|\vec{X}_{\rho}^{h}\right|\right)^{(h)}-2 \pi \varsigma \sum_{p \in \partial_{2} I \cup \partial_{F} I} \vec{X}_{t}^{h}(p) \cdot \vec{e}_{1}+2 \pi \alpha_{G} \sum_{p \in \partial_{M} I} \vec{m}_{t}^{h}(p) \cdot \vec{e}_{1} \\
& =-\pi \frac{\mathrm{d}}{\mathrm{d} t}\left(\alpha\left[\kappa_{\mathcal{S}}^{h}-\bar{\varkappa}\right]^{2}+2 \lambda, \vec{X}^{h} \cdot \vec{e}_{1}\left|\vec{X}_{\rho}^{h}\right|\right)^{(h)}-\frac{\beta}{2} \frac{\mathrm{d}}{\mathrm{d} t}\left(\mathcal{A}_{\mathcal{S}}^{h}\right)^{2}-2 \pi \varsigma \sum_{p \in \partial_{2} I \cup \partial_{F} I} \vec{X}_{t}^{h}(p) \cdot \vec{e}_{1}+2 \pi \alpha_{G} \sum_{p \in \partial_{M} I} \vec{m}_{t}^{h}(p) \cdot \vec{e}_{1} \\
& =-\frac{\mathrm{d}}{\mathrm{d} t} \widehat{E}_{\mathcal{S}}^{h}(t)
\end{aligned}
$$

where we have recalled the definition (4.30). 
In order to derive a suitable semidiscrete approximation, we now return to (4.35). Substituting (4.8) into (4.35) yields that

$$
\begin{aligned}
2 \pi\left(\left(\vec{X}^{h} \cdot \vec{e}_{1}\right) \underline{\underline{Q}^{h}} \vec{X}_{t}^{h}, \vec{\chi}\left|\vec{X}_{\rho}^{h}\right|\right)^{h}= & -\left(\pi \vec{X}^{h} \cdot \vec{e}_{1}\left[\alpha\left[\kappa_{\mathcal{S}}^{h}-\bar{\varkappa}\right]^{2}+2 \lambda+2 \beta \mathcal{A}_{\mathcal{S}}^{h} \kappa_{\mathcal{S}}^{h}\right]-\vec{Y}_{\mathcal{S}}^{h} \cdot \vec{e}_{1}, \vec{\chi}_{\rho} \cdot \vec{\tau}^{h}\right)^{(h)} \\
& -\left(\left[\pi\left[\alpha\left[\kappa_{\mathcal{S}}^{h}-\vec{\varkappa}^{2}+2 \lambda+2 \beta \mathcal{A}_{\mathcal{S}}^{h} \kappa_{\mathcal{S}}^{h}\right]-\kappa_{\mathcal{S}}^{h} \vec{Y}_{\mathcal{S}}^{h} \cdot \vec{\nu}^{h}\right]\left|\vec{X}_{\rho}^{h}\right|\right.\right. \\
& \left.-\left(\vec{Y}_{\mathcal{S}}^{h}\right)_{\rho} \cdot \vec{\tau}^{h}, \vec{\chi} \cdot \vec{e}_{1}\right)^{(h)}-\left(\vec{X}^{h} \cdot \vec{e}_{1} \kappa_{\mathcal{S}}^{h} \vec{Y}_{\mathcal{S}}^{h}, \vec{\chi}_{\rho}^{\perp}\right)^{(h)} \\
& +\left(\left(\vec{X}^{h} \cdot \vec{e}_{1}\right)\left(\vec{Y}_{\mathcal{S}}^{h}\right)_{\rho} \cdot \vec{\nu}^{h}, \vec{\chi}_{\rho} \cdot \vec{\nu}^{h}\left|\vec{X}_{\rho}^{h}\right|^{-1}\right) \\
& -\sum_{p \in \partial_{2} I \cup \partial_{F} I}\left[\left[2 \pi \varsigma+\vec{m}^{h} \cdot \vec{Y}_{\mathcal{S}}^{h}\right]\left(\vec{\chi} \cdot \vec{e}_{1}\right)\right](p) \quad \forall \vec{\chi} \in \mathbb{X}^{h} .
\end{aligned}
$$

On combining (4.40), (4.33), (4.29), (4.34) and (4.31), on noting (3.1a) and (4.4), our semidiscrete approximation based on $\kappa_{\mathcal{S}}^{h}$ is given as follows.

$\left(\mathcal{P}_{\mathcal{S}}^{h}\right)^{(h)}$ Let $\vec{X}^{h}(\cdot, 0) \in \underline{V}_{\partial_{0}}^{h}$ and $\alpha \in \mathbb{R}_{>0}, \bar{\varkappa}, M_{0}, \alpha_{G} \lambda, \varsigma \in \mathbb{R}, \beta \in \mathbb{R}_{\geq 0}, \vec{\zeta}: \partial_{C} I \rightarrow \mathbb{S}^{1}$ be given. For $t \in(0, T]$ find $\vec{X}^{h}(\cdot, t)$, with $\vec{X}_{t}^{h}(\cdot, t) \in \mathbb{X}^{h}, \kappa_{\mathcal{S}}^{h}(\cdot, t) \in W_{\left(\partial_{0}\right)}^{h}, \vec{Y}_{\mathcal{S}}^{h}(\cdot, t) \in \underline{V}_{\partial_{0}}^{h}$, with $\vec{\pi}^{h}\left[\left(\vec{X}^{h} \cdot \vec{e}_{1}\right) \vec{Y}_{\mathcal{S}}^{h}\right](\cdot, t) \in \mathbb{Y}^{h}\left(2 \pi \alpha_{G} \vec{e}_{1}\right)$, and $\vec{m}^{h}(\cdot, t): \partial_{M} I \rightarrow \mathbb{R}^{2}$ such that

$$
\begin{aligned}
& 2 \pi\left(\left(\vec{X}^{h} \cdot \vec{e}_{1}\right) \underline{\underline{Q}}^{h} \vec{X}_{t}^{h}, \vec{\chi}\left|\vec{X}_{\rho}^{h}\right|\right)^{h}-\left(\left(\vec{X}^{h} \cdot \vec{e}_{1}\right)\left(\vec{Y}_{\mathcal{S}}^{h}\right)_{\rho} \cdot \vec{\nu}^{h}, \vec{\chi}_{\rho} \cdot \vec{\nu}^{h}\left|\vec{X}_{\rho}^{h}\right|^{-1}\right) \\
& =-\left(\pi \vec{X}^{h} \cdot \vec{e}_{1}\left[\alpha\left[\kappa_{\mathcal{S}}^{h}-\bar{\varkappa}\right]^{2}+2 \lambda+2 \beta \mathcal{A}_{\mathcal{S}}^{h} \kappa_{\mathcal{S}}^{h}\right]-\vec{Y}_{\mathcal{S}}^{h} \cdot \vec{e}_{1}, \vec{\chi}_{\rho} \cdot \vec{\tau}^{h}\right)^{(h)} \\
& \quad-\left(\left[\pi\left[\alpha\left[\kappa_{\mathcal{S}}^{h}-\bar{\varkappa}\right]^{2}+2 \lambda+2 \beta \mathcal{A}_{\mathcal{S}}^{h} \kappa_{\mathcal{S}}^{h}\right]-\kappa_{\mathcal{S}}^{h} \vec{Y}_{\mathcal{S}}^{h} \cdot \vec{\nu}^{h}\right]\left|\vec{X}_{\rho}^{h}\right|-\left(\vec{Y}_{\mathcal{S}}^{h}\right)_{\rho} \cdot \vec{\tau}^{h}, \vec{\chi} \cdot \vec{e}_{1}\right)^{(h)} \\
& \quad+\left(\vec{X}^{h} \cdot \vec{e}_{1} \kappa_{\mathcal{S}}^{h}\left(\vec{Y}_{\mathcal{S}}^{h}\right)^{\perp}, \vec{\chi}_{\rho}\right)^{(h)}-\sum_{p \in \partial_{2} I \cup \partial_{F} I}\left[\left[2 \pi \varsigma+\vec{m}^{h} \cdot \vec{Y}_{\mathcal{S}}^{h}\right]\left(\vec{\chi} \cdot \vec{e}_{1}\right)\right](p) \quad \forall \vec{\chi} \in \mathbb{X}^{h}, \\
& 2 \pi\left(\vec{X}^{h} \cdot \vec{e}_{1}\left[\alpha\left(\kappa_{\mathcal{S}}^{h}-\bar{\varkappa}\right)+\beta \mathcal{A}_{\mathcal{S}}^{h}\right], \chi\left|\vec{X}_{\rho}^{h}\right|\right)^{(h)}-\left(\left(\vec{X}^{h} \cdot \vec{e}_{1}\right) \vec{Y}_{\mathcal{S}}^{h}, \chi \vec{\nu}^{h}\left|\vec{X}_{\rho}^{h}\right|\right)^{(h)}=0 \quad \forall \chi \in W_{\left(\partial_{0}\right)}^{h}, \\
& \left(\vec{X}^{h} \cdot \vec{e}_{1} \kappa_{\mathcal{S}}^{h} \vec{\nu}^{h}, \vec{\eta}\left|\vec{X}_{\rho}^{h}\right|\right)^{(h)}+\left(\vec{e}_{1}, \vec{\eta}\left|\vec{X}_{\rho}^{h}\right|\right)+\left(\left(\vec{X}^{h} \cdot \vec{e}_{1}\right) \vec{X}_{\rho}^{h}, \vec{\eta}_{\rho}\left|\vec{X}_{\rho}^{h}\right|^{-1}\right) \\
& \quad=\sum_{p \in \partial_{C} I}\left[\left(\vec{X}^{h} \cdot \vec{e}_{1}\right) \vec{\zeta} \cdot \vec{\eta}\right](p)+\sum_{p \in \partial_{M} I}\left[\left(\vec{X}^{h} \cdot \vec{e}_{1}\right) \vec{m}^{h} \cdot \vec{\eta}\right](p) \quad \forall \vec{\eta} \in V_{\partial_{0}}^{h},
\end{aligned}
$$

where $\mathcal{A}_{\mathcal{S}}^{h}(t)$ is given by $(4.31)$.

Theorem 4.8. Let Assumption 4.2 be satisfied and let $\left(\vec{X}^{h}(t), \kappa_{\mathcal{S}}^{h}(t), \vec{Y}_{\mathcal{S}}^{h}(t), \vec{m}^{h}(t)\right)_{t \in(0, T]}$ be a solution to (4.41). Then the solution satisfies the stability bound

$$
\frac{\mathrm{d}}{\mathrm{d} t} \widehat{E}_{\mathcal{S}}^{h}(t)+2 \pi\left(\vec{X}^{h} \cdot \vec{e}_{1}\left|\underline{\underline{Q}}^{h} \vec{X}_{t}^{h}\right|^{2},\left|\vec{X}_{\rho}^{h}\right|\right)^{h}=0 .
$$

Proof. The desired result follows as (4.41) is just a rewrite of (4.35), (4.33), (4.29) and (4.31), and then noting $(4.36)-(4.39)$.

Remark 4.9. For the scheme $\left(\mathcal{P}_{\mathcal{S}}^{h}\right)^{h}$ we note that if $0 \in \partial_{0} I$, then choosing $\vec{\eta}=\chi_{0} \vec{e}_{2}$ in (4.41c) yields that $\left(\vec{X}^{h}\left(q_{1}\right)-\vec{X}^{h}\left(q_{0}\right)\right) \cdot \vec{e}_{2}=0$, recall also Remark 4.6. While the same is not true for the scheme $\left(\mathcal{P}_{\mathcal{S}}^{h}\right)$, a weaker form of these constraints is still enforced via (4.41c), leading to a nearly $90^{\circ}$ degree contact angle on the $x_{2}$-axis in practice for fully discrete variants based on $\left(\mathcal{P}_{\mathcal{S}}^{h}\right)$. 


\section{Fully Discrete SCHEME}

Let $0=t_{0}<t_{1}<\ldots<t_{M-1}<t_{M}=T$ be a partitioning of $[0, T]$ into possibly variable time steps $\Delta t_{m}=t_{m+1}-t_{m}, m=0, \ldots, M-1$. For $\vec{X}^{m} \in \underline{V}_{\partial_{0}}^{h}$, we let $\vec{\tau}^{m}$ and $\vec{\nu}^{m}$ be the natural fully discrete analogues of $\vec{\tau}^{h}$ and $\vec{\nu}^{h}$ on $\Gamma^{m}=\vec{X}^{m}(I)$, recall (4.4). In addition, let $\vec{\omega}^{m} \in \underline{V}^{h}$ be the natural fully discrete analogue of $\vec{\omega}^{h} \in \underline{V}^{h}$, recall (4.5); and similarly for $\vec{v}^{m} \in \underline{V}^{h}$, recall (4.6). Finally, let $\vec{Q}^{m} \in\left[V^{h}\right]^{2 \times 2}$ be the natural fully discrete analogue of $\vec{Q}^{h}$, recall (4.7).

\subsection{Based on $\kappa^{m+1}$}

We propose the following fully discrete approximation of $\left(\mathcal{P}^{h}\right)^{h}$.

$\left(\mathcal{P}^{m}\right)^{h}$ Let $\vec{X}^{0} \in \underline{V}_{\partial_{0}}^{h}, \kappa^{0} \in V^{h}, \vec{Y}^{0} \in \underline{V}_{\partial_{0}}^{h}$ and $\alpha \in \mathbb{R}_{>0}, \bar{\varkappa}, M_{0}, \alpha_{G}, \lambda, \varsigma \in \mathbb{R}, \beta \in \mathbb{R}_{\geq 0}, \vec{\zeta}: \partial_{C} I \rightarrow \mathbb{S}^{1}$ be given. For $m=0, \ldots, M-1$, find $\delta \vec{X}^{m+1} \in \mathbb{X}^{h}$, with $\vec{X}^{m+1}=\vec{X}^{m}+\delta \vec{X}^{m+1}, \kappa^{m+1} \in W_{\partial_{0}}^{h}, \vec{Y}^{m+1} \in \mathbb{Y}^{h}\left(2 \pi \alpha_{G} \vec{e}_{1}\right)$ and $\vec{m}^{m+1}: \partial_{M} I \rightarrow \mathbb{R}^{2}$ such that

$$
\begin{aligned}
& 2 \pi\left(\vec{X}^{m} \cdot \vec{e}_{1} \underline{\underline{Q}}^{m} \frac{\vec{X}^{m+1}-\vec{X}^{m}}{\Delta t_{m}}, \vec{\chi}\left|\vec{X}_{\rho}^{m}\right|\right)^{h}-\left(\vec{Y}_{\rho}^{m+1}, \vec{\chi}_{\rho}\left|\vec{X}_{\rho}^{m}\right|^{-1}\right)+\left(\vec{Y}_{\rho}^{m} \cdot \vec{\tau}^{m}, \vec{\chi}_{\rho} \cdot \vec{\tau}^{m}\left|\vec{X}_{\rho}^{m}\right|^{-1}\right) \\
& =-\pi\left(\alpha\left[\mathfrak{K}^{h}\left(\vec{X}^{m}, \kappa^{m}\right)-\bar{\varkappa}\right]^{2}+2 \lambda+2 \beta \mathcal{A}^{m} \kappa^{m}, \vec{\chi} \cdot \vec{e}_{1}\left|\vec{X}_{\rho}^{m}\right|+\left(\vec{X}^{m} \cdot \vec{e}_{1}\right) \vec{\tau}^{m} \cdot \vec{\chi}_{\rho}\right)^{h} \\
& +2 \pi \alpha\left(\left[\mathfrak{K}^{h}\left(\vec{X}^{m}, \kappa^{m}\right)-\bar{\varkappa}\right]\left(\mathfrak{Z}^{h}-2\right), \frac{\vec{\omega}^{m} \cdot \vec{e}_{1}}{\vec{X}^{m} \cdot \vec{e}_{1}} \vec{\chi} \cdot \vec{e}_{1}\left|\vec{X}_{\rho}^{m}\right|\right)^{h} \\
& +2 \pi \alpha\left(\left[\mathfrak{K}^{h}\left(\vec{X}^{m}, \kappa^{m}\right)-\bar{\varkappa}\right]\left(\mathfrak{Z}^{h}-2\right) \vec{e}_{1},\left(\vec{\nu}^{m} \cdot \vec{\chi}_{\rho}\right) \vec{\tau}^{m}+\left(\vec{\tau}^{m} \cdot \vec{\chi}_{\rho}\right)\left(\vec{\omega}^{m}-\vec{\nu}^{m}\right)\right)^{h} \\
& +\left(\kappa^{m}\left(\vec{Y}^{m}\right)^{\perp}-2 \pi \beta \mathcal{A}^{m} \vec{e}_{2}, \vec{\chi}_{\rho}\right)^{h}-2 \pi \varsigma \sum_{p \in \partial_{2} I \cup \partial_{F} I} \vec{\chi}(p) \cdot \vec{e}_{1} \quad \forall \vec{\chi} \in \mathbb{X}^{h}, \\
& 2 \pi\left(\vec{X}^{m} \cdot \vec{e}_{1}\left(\alpha\left[\mathfrak{K}^{h}\left(\vec{X}^{m}, \kappa^{m+1}\right)-\bar{\varkappa}\right]+\beta \mathcal{A}^{m}\right), \chi\left|\vec{X}_{\rho}^{m}\right|\right)^{h}-\left(\vec{Y}^{m+1}, \chi \vec{\nu}^{m}\left|\vec{X}_{\rho}^{m}\right|\right)^{h}=0 \quad \forall \chi \in W_{\partial_{0}}^{h}, \\
& \left(\kappa^{m+1} \vec{\nu}^{m}, \vec{\eta}\left|\vec{X}_{\rho}^{m}\right|\right)^{h}+\left(\vec{X}_{\rho}^{m+1}, \vec{\eta}_{\rho}\left|\vec{X}_{\rho}^{m}\right|^{-1}\right)=\sum_{p \in \partial_{C} I}[\vec{\zeta} \cdot \vec{\eta}](p)+\sum_{p \in \partial_{M} I}\left[\vec{m}^{m+1} \cdot \vec{\eta}\right](p) \quad \forall \vec{\eta} \in \underline{V}_{\partial_{0}}^{h},
\end{aligned}
$$

where

$$
\mathcal{A}^{m}=2 \pi\left(\vec{X}^{m} \cdot \vec{e}_{1} \kappa^{m}-\vec{\nu}^{m} \cdot \vec{e}_{1},\left|\vec{X}_{\rho}^{m}\right|\right)^{h}-M_{0} .
$$

Assumption 5.1. Let $\vec{X}^{m}$ satisfy Assumption 4.2 with $\vec{X}^{h}$ replaced by $\vec{X}^{m}$, and let $\operatorname{dim} \operatorname{span}\left\{\vec{v}^{m}\left(q_{j}\right)\right\}_{j=1}^{J-1}=2$.

Assumption 5.2. Let $\vec{X}^{m}$ satisfy Assumption 4.1 with $\vec{X}^{h}$ replaced by $\vec{X}^{m}$, and be such that the following holds. If $\vec{U} \in \mathbb{Y}^{h}(\overrightarrow{0})$ with

$$
\left(\vec{U}_{\rho}, \vec{\chi}_{\rho}\left|\vec{X}_{\rho}^{m}\right|^{-1}\right)=0 \quad \forall \vec{\chi} \in \mathbb{X}^{h} \quad \text { and } \quad\left(\vec{U}, \chi \vec{\nu}^{m}\left|\vec{X}_{\rho}^{m}\right|\right)^{h}=0 \quad \forall \chi \in W_{\partial_{0}}^{h},
$$

then $\vec{U}=\overrightarrow{0}$.

We note that Assumption 5.2 is only violated in very rare cases. For example, if $\emptyset \neq \partial_{C} I=\partial I$ and if $\vec{X}^{m}$ parameterizes a straight line, then $\vec{U}=\vec{\tau}^{m}$ constant in $I$ satisfies (5.2). However, the following lemma shows that in most cases the assumption holds.

Lemma 5.3. Let $\vec{X}^{m}$ satisfy Assumption 5.1. Then if $\partial_{C} I=\emptyset$, or if $\partial_{C} I \neq \partial I \backslash \partial_{0} I$, then Assumption 5.2 holds. 
Proof. Let $\vec{U} \in \mathbb{Y}^{h}(\overrightarrow{0})$ satisfy (5.2). If $\partial_{C} I=\emptyset$ then we can choose $\vec{\chi}=\vec{U} \in \mathbb{Y}^{h}(\overrightarrow{0}) \subset \mathbb{X}^{h}$ in (5.2), recall (3.7), to obtain that $\vec{U}$ is constant in $\bar{I}$. The second property in (5.2), on recalling (4.5), then yields that $\vec{U} \cdot \vec{\omega}^{m}\left(q_{j}\right)=\vec{U} \cdot \vec{v}^{m}\left(q_{j}\right)=0, j=1, \ldots, J-1$. Hence Assumption 5.1 gives that $\vec{U}=\overrightarrow{0}$.

We now consider the case $\partial_{C} I \neq \emptyset$. As we assume $\partial_{C} I \neq \partial I \backslash \partial_{0} I$, it holds that also $\partial_{M} I \neq \emptyset$, recall (2.37). For ease of exposition, let $\partial_{M} I=\{0\}$ and $\partial_{C} I=\{1\}$. It follows from the first condition in (5.2) that

$$
\frac{\vec{U}\left(q_{j+1}\right)-\vec{U}\left(q_{j}\right)}{\left|\vec{X}^{m}\left(q_{j+1}\right)-\vec{X}^{m}\left(q_{j}\right)\right|}=\frac{\vec{U}\left(q_{j}\right)-\vec{U}\left(q_{j-1}\right)}{\left|\vec{X}^{m}\left(q_{j}\right)-\vec{X}^{m}\left(q_{j-1}\right)\right|}, \quad j=1, \ldots, J-1,
$$

and so, on recalling Assumption 5.1, there exist positive numbers $\alpha_{j}$ such that

$$
\vec{U}\left(q_{j+1}\right)=\left(1+\alpha_{j}\right) \vec{U}\left(q_{j}\right)-\alpha_{j} \vec{U}\left(q_{j-1}\right), \quad j=1, \ldots, J-1 .
$$

Combining (5.3) and the fact that $\vec{U} \in \mathbb{Y}^{h}(\overrightarrow{0})$, i.e. $\vec{U}\left(q_{0}\right)=\overrightarrow{0}$, we obtain, via induction, that there exist numbers $\zeta_{J-1} \geq \zeta_{J-2} \geq \ldots \geq \zeta_{1}>0$ such that

$$
\vec{U}\left(q_{j+1}\right)=\left(1+\zeta_{j}\right) \vec{U}\left(q_{1}\right), \quad j=1, \ldots, J-1 .
$$

Hence it follows from the second property in (5.2) and the Assumption 5.1, recall (4.5), that $\vec{U}\left(q_{1}\right)=\overrightarrow{0}$, and so $\vec{U}=\overrightarrow{0}$ in $I$. This completes the proof.

Lemma 5.4. Let Assumption 5.1 hold. Moreover, if $\emptyset \neq \partial_{C} I$ and if $\partial_{C} I=\partial I \backslash \partial_{0} I$ then let also Assumption 5.2 hold. Let $\vec{X}^{m}, \vec{Y}^{m} \in \underline{V}_{\partial_{0}}^{h}, \kappa^{m} \in V^{h}, \vec{m}^{m} \in \mathbb{R}^{2}$ and $\alpha \in \mathbb{R}_{>0}, \bar{\varkappa}, M_{0}, \alpha_{G} \in \mathbb{R}, \beta \in \mathbb{R}_{\geq 0}, \vec{\zeta}: \partial_{C} I \rightarrow \mathbb{R}^{2}$ be given. Then there exists a unique solution to $\left(\mathcal{P}^{m}\right)^{h},(5.1)$.

Proof. As we have a linear system of equations, with the same number of equations as unknowns, existence follows from uniqueness. Hence we consider a solution to the homogeneous equivalent of (5.1), and need to show that this solution is in fact zero. In particular, let $\delta \vec{X} \in \mathbb{X}^{h}, \kappa \in W_{\partial_{0}}^{h}, \vec{Y} \in \mathbb{Y}^{h}(\overrightarrow{0})$, and $\vec{m}: \partial_{M} I \rightarrow \mathbb{R}^{2}$ be such that

$$
\begin{aligned}
2 \pi\left(\left(\vec{X}^{m} \cdot \vec{e}_{1}\right) \underline{\underline{Q}}^{m} \delta \vec{X}, \vec{\chi}\left|\vec{X}_{\rho}^{m}\right|\right)^{h}-\Delta t_{m}\left(\vec{Y}_{\rho}, \vec{\chi}_{\rho}\left|\vec{X}_{\rho}^{m}\right|^{-1}\right) & =0 & & \forall \vec{\chi} \in \mathbb{X}^{h}, \\
2 \pi \alpha\left(\vec{X}^{m} \cdot \vec{e}_{1} \kappa, \chi\left|\vec{X}_{\rho}^{m}\right|\right)^{h}-\left(\vec{Y}, \chi \vec{\nu}^{m}\left|\vec{X}_{\rho}^{m}\right|\right)^{h} & =0 & & \forall \chi \in W_{\partial_{0}}^{h}, \\
\left(\kappa \vec{\nu}^{m}, \vec{\eta}\left|\vec{X}_{\rho}^{m}\right|\right)^{h}+\left((\delta \vec{X})_{\rho}, \vec{\eta}_{\rho}\left|\vec{X}_{\rho}^{m}\right|^{-1}\right) & =\sum_{p \in \partial_{M} I}[\vec{m} \cdot \vec{\eta}](p) & & \forall \vec{\eta} \in \underline{V}_{\partial_{0}}^{h} .
\end{aligned}
$$

Choosing $\vec{\chi}=\delta \vec{X}$ in (5.4a), $\chi=\kappa$ in (5.4b) and $\vec{\eta}=\vec{Y}$ in (5.4c) yields that

$$
2 \pi\left(\vec{X}^{m} \cdot \vec{e}_{1}\left|\underline{\underline{Q}}^{m} \delta \vec{X}\right|^{2},\left|\vec{X}_{\rho}^{m}\right|\right)^{h}+2 \pi \alpha \Delta t_{m}\left(\vec{X}^{m} \cdot \vec{e}_{1} \kappa^{2},\left|\vec{X}_{\rho}^{m}\right|\right)^{h}=0 .
$$

It follows from (5.5) and $\kappa \in W_{\partial_{0}}^{h}$ that $\kappa=0$. Similarly, it follows from (5.5), $\delta \vec{X} \in \underline{V}_{\partial_{0}}^{h},(3.5 \mathrm{a}),(4.6)$ and (4.7) that $\pi^{h}\left[\delta \vec{X} \cdot \vec{v}^{m}\right]=0$ with $\delta \vec{X}=\overrightarrow{0}$ on $\partial I \backslash \partial_{0} I$. Then choosing $\vec{\eta}=\delta \vec{X} \in \mathbb{X}^{h} \subset \underline{V}_{\partial_{0}}^{h}$ in (5.4c) yields, on recalling (2.37) and $\delta \vec{X}=\overrightarrow{0}$ on $\partial I \backslash \partial_{0} I$, that

$$
\left(\left|(\delta \vec{X})_{\rho}\right|^{2},\left|\vec{X}_{\rho}^{m}\right|^{-1}\right)=\sum_{p \in \partial_{M} I}[\vec{m} \cdot \delta \vec{X}](p)=0 .
$$

It follows from (5.6) that $\delta \vec{X}$ is constant in $\bar{I}$. Together with $\pi^{h}\left[\delta \vec{X} \cdot \vec{v}^{m}\right]=0$ and Assumption 5.1 we obtain that $\delta \vec{X}=\overrightarrow{0}$. Now (5.4c) implies that $\vec{m}=\overrightarrow{0}$. 
It remains to show that $\vec{Y}=\overrightarrow{0}$. We have from (5.4a) and (5.4b) that

$$
\left(\vec{Y}_{\rho}, \vec{\chi}_{\rho}\left|\vec{X}_{\rho}^{m}\right|^{-1}\right)=0 \quad \forall \vec{\chi} \in \mathbb{X}^{h} \quad \text { and } \quad\left(\vec{Y}, \chi \vec{\nu}^{m}\left|\vec{X}_{\rho}^{m}\right|\right)^{h}=0 \quad \forall \chi \in W_{\partial_{0}}^{h}
$$

Moreover, our assumptions and Lemma 5.3 yield that Assumption 5.2 holds. Hence we have that $\vec{Y}=\overrightarrow{0}$, and thus we have shown the existence of a unique solution to $\left(\mathcal{P}^{m}\right)^{h}$.

Remark 5.5. We note that it is not possible to prove the existence of a unique solution to (5.1) in the case of clamped boundary conditions, when $\partial_{C} I=\partial I=\{0,1\}$. The authors faced a similar issue in the context of the approximation of Willmore flow for general open surfaces in [6]. There the problem could be overcome by a suitable tweak to the discretization, see (3.19) and Theorem 4.1 there. In particular, the approach applied in [6] relied on the discretization of the side constraint (2.22) there, which is formulated in terms of the mean curvature vector $\vec{k}_{m}=k_{m} \vec{n}_{\mathcal{S}}$ of $\mathcal{S}$, rather than in terms of the scalar curvature variables $\varkappa$ and $\varkappa_{\mathcal{S}}$ that we consider here, recall (3.8) and (3.9). Hence the approach from [6] is not applicable to the situation considered in this paper.

Remark 5.6. We note that in practice it is easiest to find the solution to (5.1) by first eliminating $\vec{m}^{m+1}$ from (5.1) via replacing the test space $\underline{V}_{\partial_{0}}^{h}$ in (5.1c) with $\mathbb{Y}^{h}(\overrightarrow{0})$. Having computed $\left(\delta \vec{X}^{m+1}, \kappa^{m+1}, \vec{Y}^{m+1}\right)$ in this way, for example with the help of a sparse factorization package like UMFPACK, see [18], the values $\vec{m}^{m+1}$ can be obtained from (5.1c). For example, if $q_{0} \in \partial_{M} I$ then we have, on recalling (4.5), that

$$
\vec{m}^{m+1}\left(q_{0}\right)=\left(1, \chi_{0}\left|\vec{X}_{\rho}^{m}\right|\right) \kappa^{m+1}\left(q_{0}\right) \vec{\omega}^{m}\left(q_{0}\right)+\left(1,\left(\chi_{0}\right)_{\rho}\left|\vec{X}_{\rho}^{m}\right|^{-1}\right) \vec{X}_{\rho}^{m+1}\left(q_{0}\right) .
$$

Remark 5.7. In light of our stability result Theorem 4.4 for the semidiscrete scheme (4.23), it would be desirable to also prove (conditional) stability for the fully discrete scheme (5.1). However, at present this remains an open problem, in line with other fully discrete schemes for Willmore flow and elastic flow in the literature, see e.g. $[2,4,5,19,21]$. Note that the only stability result for a fully discrete scheme for Willmore flow, that we are aware of, is given in [32], for a scheme where the tangential velocity is zero.

\subsubsection{Conserved flows}

Here, following the approach in Section 4.3.1 of [7], we consider fully discrete variants of the semidiscrete conserving approximations in Section 4.1.1. In particular, on rewriting (5.1a) as

$$
2 \pi\left(\vec{X}^{m} \cdot \vec{e}_{1} \underline{\underline{Q}}^{m} \frac{\vec{X}^{m+1}-\vec{X}^{m}}{\Delta t_{m}}, \vec{\chi}\left|\vec{X}_{\rho}^{m}\right|\right)^{h}-\left(\vec{Y}_{\rho}^{m+1}, \vec{\chi}_{\rho}\left|\vec{X}_{\rho}^{m}\right|^{-1}\right)=\left(\vec{f}^{m}, \vec{\chi}\left|\vec{X}_{\rho}^{m}\right|\right)^{h}
$$

we can formulate our surface area and volume conserving variant for $\left(\mathcal{P}^{m}\right)^{h}$ as follows. Here, for ease of presentation, we assume that $\partial_{M} I=\emptyset$, so that we do not need to consider the discrete conormals $\vec{m}^{m+1}$. Moreover, on recalling (3.6), we have that $\mathbb{Y}^{h}\left(2 \pi \alpha_{G} \vec{e}_{1}\right)=\underline{V}_{\partial_{0}}^{h}$.

$\left(\mathcal{P}_{A, V}^{m}\right)^{h}:$ Let $\vec{X}^{0} \in \underline{V}_{\partial_{0}}^{h}, \kappa^{0} \in V^{h}, \vec{Y}^{0} \in \underline{V}_{\partial_{0}}^{h}$ and $\alpha \in \mathbb{R}_{>0}, \bar{\varkappa}, M_{0}, \alpha_{G}, \lambda, \varsigma \in \mathbb{R}, \beta \in \mathbb{R}_{\geq 0}, \vec{\zeta}: \partial_{C} I \rightarrow \mathbb{S}^{1}$ be given. For $m=0, \ldots, M-1$, find $\delta \vec{X}^{m+1} \in \mathbb{X}^{h}$, with $\vec{X}^{m+1}=\vec{X}^{m}+\delta \vec{X}^{m+1}, \kappa^{m+1} \in W_{\partial_{0}}^{h}, \vec{Y}^{m+1} \in \underline{V}_{\partial_{0}}^{h}$, and $\lambda_{A}^{m+1}, \lambda_{V}^{m+1} \in \mathbb{R}$ such that (5.1b), (5.1c) and

$$
\begin{aligned}
& 2 \pi\left(\vec{X}^{m} \cdot \vec{e}_{1} \underline{\underline{Q}}^{m} \frac{\vec{X}^{m+1}-\vec{X}^{m}}{\Delta t_{m}}, \vec{\chi}\left|\vec{X}_{\rho}^{m}\right|\right)^{h}-\left(\vec{Y}_{\rho}^{m+1}, \vec{\chi}_{\rho}\left|\vec{X}_{\rho}^{m}\right|^{-1}\right)=\left(\vec{f}^{m}, \vec{\chi}\left|\vec{X}_{\rho}^{m}\right|\right)^{h} \\
& \quad-2 \pi \lambda_{A}^{m+1}\left[\left(\vec{e}_{1}, \vec{\chi}\left|\vec{X}_{\rho}^{m}\right|\right)+\left(\left(\vec{X}^{m} \cdot \vec{e}_{1}\right) \vec{\tau}^{m}, \vec{\chi}_{\rho}\right)\right]-2 \pi \lambda_{V}^{m+1}\left(\left(\vec{X}^{m} \cdot \vec{e}_{1}\right) \vec{\nu}^{m}, \vec{\chi}\left|\vec{X}_{\rho}^{m}\right|\right) \quad \forall \vec{\chi} \in \mathbb{X}^{h}, \\
& \text { (i) } A\left(\vec{X}^{m+1}\right)=A\left(\vec{X}^{0}\right), \quad \text { (ii) } V\left(\vec{X}^{m+1}\right)=V\left(\vec{X}^{0}\right)
\end{aligned}
$$


hold. The nonlinear system of equations arising at each time level of $\left(\mathcal{P}_{A, V}^{m}\right)^{h}$ can be solved with a suitable iterative solution method, see below. In the simpler case of surface area conserving flow, we need to find $\left(\delta \vec{X}^{m+1}, \kappa^{m+1}, \vec{Y}^{m+1}, \lambda_{A}^{m+1}, \lambda_{V}^{m+1}\right) \in \mathbb{X}^{h} \times W_{\partial_{0}}^{h} \times \underline{V}_{\partial_{0}}^{h} \times \mathbb{R} \times\{0\}$ such that (5.1b), (5.1c), (5.7a) and (5.7b)(i) hold. Similarly, for volume conserving flow, we need to find $\left(\delta \vec{X}^{m+1}, \kappa^{m+1}, \vec{Y}^{m+1}, \lambda_{A}^{m+1}, \lambda_{V}^{m+1}\right) \in \mathbb{X}^{h} \times W_{\partial_{0}}^{h} \times$ $\underline{V}_{\partial_{0}}^{h} \times\{0\} \times \mathbb{R}$ such that (5.1b), (5.1c), (5.7a) and (5.7b)(ii) hold.

Adapting the strategy in Section 4.3.1 of [7], we now describe a Newton method for solving the nonlinear system (5.7), (5.1b) and (5.1c). The linear system (5.7a), (5.1b) and (5.1c), with $\left(\lambda_{A}^{m+1}, \lambda_{V}^{m+1}\right)$ in (5.7a) replaced by $\left(\lambda_{A}, \lambda_{V}\right)$, can be written as: Find $\left(\delta \vec{X}^{m+1}\left(\lambda_{A}, \lambda_{V}\right), \kappa^{m+1}\left(\lambda_{A}, \lambda_{V}\right), \vec{Y}^{m+1}\left(\lambda_{A}, \lambda_{V}\right)\right) \in \mathbb{X}^{h} \times W_{\partial_{0}}^{h} \times \underline{V}_{\partial_{0}}^{h}$ such that

$$
\mathbb{T}^{m}\left(\begin{array}{c}
\vec{Y}^{m+1}\left(\lambda_{A}, \lambda_{V}\right) \\
\delta \vec{X}^{m+1}\left(\lambda_{A}, \lambda_{V}\right) \\
\kappa^{m+1}\left(\lambda_{A}, \lambda_{V}\right)
\end{array}\right)=\left(\begin{array}{c}
\overrightarrow{\mathfrak{g}}_{1}^{m} \\
\overrightarrow{\mathfrak{g}}_{2}^{m} \\
\overrightarrow{\mathfrak{g}}_{3}^{m}
\end{array}\right)+\lambda_{A}\left(\begin{array}{c}
\underline{\overrightarrow{\mathcal{K}}}^{m} \\
0 \\
\overrightarrow{0}
\end{array}\right)+\lambda_{V}\left(\begin{array}{c}
\underline{\overrightarrow{\mathcal{N}}}^{m} \\
0 \\
\overrightarrow{0}
\end{array}\right)
$$

Assuming the linear operator $\mathbb{T}^{m}$ is invertible, we obtain that

$$
\left(\begin{array}{c}
\vec{Y}^{m+1}\left(\lambda_{A}, \lambda_{V}\right) \\
\delta \vec{X}^{m+1}\left(\lambda_{A}, \lambda_{V}\right) \\
\kappa^{m+1}\left(\lambda_{A}, \lambda_{V}\right)
\end{array}\right)=\left(\mathbb{T}^{m}\right)^{-1}\left[\left(\begin{array}{c}
\overrightarrow{\mathfrak{g}}_{1}^{m} \\
\overrightarrow{\mathfrak{g}}_{2}^{m} \\
\underline{\mathfrak{g}}_{3}^{m}
\end{array}\right)+\lambda_{V}\left(\begin{array}{c}
\underline{\mathcal{N}}^{m} \\
0 \\
\overrightarrow{0}
\end{array}\right)\right]=:\left(\mathbb{T}^{m}\right)^{-1}\left(\begin{array}{c}
\underline{\overrightarrow{\mathfrak{g}}}_{1}^{m} \\
\underline{\mathfrak{g}}_{2}^{m} \\
\underline{\mathfrak{g}}_{3}^{m}
\end{array}\right)+\lambda_{A}\left(\begin{array}{c}
\underline{\vec{s}}_{1}^{m} \\
\underline{\vec{s}}_{2}^{m} \\
\underline{s}_{3}^{m}
\end{array}\right)+\lambda_{V}\left(\begin{array}{c}
\vec{q}_{1}^{m} \\
\overrightarrow{\vec{q}}_{2}^{m} \\
\underline{q}_{3}^{m}
\end{array}\right) .
$$

It immediately follows from (5.8) that

$$
\partial_{\lambda_{A}} \vec{X}^{m+1}\left(\lambda_{A}, \lambda_{V}\right)=\underline{\vec{s}}_{2}^{m}, \quad \partial_{\lambda_{V}} \vec{X}^{m+1}\left(\lambda_{A}, \lambda_{V}\right)=\underline{\vec{q}}_{2}^{m},
$$

where $\vec{X}^{m+1}\left(\lambda_{A}, \lambda_{V}\right)=\vec{X}^{m}+\delta \vec{X}^{m+1}\left(\lambda_{A}, \lambda_{V}\right)$. Hence we can proceed as in (4.13) of [7] to define a Newton iteration for finding a solution to the nonlinear system $\left(\mathcal{P}_{A, V}^{m}\right)^{h}$. In practice this Newton iteration always converged within a couple of iterations.

\subsection{Based on $\kappa_{\mathcal{S}}^{m+1}$}

We can consider the following fully discrete approximation of (4.41).

$\left(\mathcal{P}_{\mathcal{S}}^{m}\right)^{(h)}$ Let $\vec{X}^{0}, \vec{Y}_{\mathcal{S}}^{0} \in \underline{V}_{\partial_{0}}^{h}, \kappa_{\mathcal{S}}^{0} \in V^{h}, \vec{m}^{0} \in \mathbb{R}^{2}$ and $\alpha \in \mathbb{R}_{>0}, \bar{\varkappa}, M_{0}, \alpha_{G}, \lambda, \varsigma \in \mathbb{R}, \beta \in \mathbb{R}_{\geq 0}, \vec{\zeta}: \partial_{C} I \rightarrow \mathbb{S}^{1}$ be given. For $m=0, \ldots, M-1$, find $\delta \vec{X}^{m+1} \in \mathbb{X}^{h}$, with $\vec{X}^{m+1}=\vec{X}^{m}+\delta \vec{X}^{m+1}, \kappa_{\mathcal{S}}^{m+1} \in W_{\left(\partial_{0}\right)}^{h}, \vec{Y}_{\mathcal{S}}^{m+1} \in \underline{V}_{\partial_{0}}^{h}$, with $\vec{\pi}^{h}\left[\left(\vec{X}^{m} \cdot \vec{e}_{1}\right) \vec{Y}_{\mathcal{S}}^{m+1}\right] \in \mathbb{Y}^{h}\left(2 \pi \alpha_{G} \vec{e}_{1}\right)$, and $\vec{m}^{m+1}: \partial_{M} I \rightarrow \mathbb{R}^{2}$ such that

$$
\begin{aligned}
& 2 \pi\left(\left(\vec{X}^{m} \cdot \vec{e}_{1}\right) \underline{\underline{Q}}^{m} \frac{\vec{X}^{m+1}-\vec{X}^{m}}{\Delta t_{m}}, \vec{\chi}\left|\vec{X}_{\rho}^{m}\right|\right)^{h}-\left(\left(\vec{X}^{m} \cdot \vec{e}_{1}\right)\left(\vec{Y}_{\mathcal{S}}^{m+1}\right)_{\rho}, \vec{\chi}_{\rho}\left|\vec{X}_{\rho}^{m}\right|^{-1}\right) \\
&+\left(\left(\vec{X}^{m} \cdot \vec{e}_{1}\right)\left(\vec{Y}_{\mathcal{S}}^{m}\right)_{\rho} \cdot \vec{\tau}^{m}, \vec{\chi}_{\rho} \cdot \vec{\tau}^{m}\left|\vec{X}_{\rho}^{m}\right|^{-1}\right) \\
&=-\left(\pi \vec{X}^{m} \cdot \vec{e}_{1}\left[\alpha\left[\kappa_{\mathcal{S}}^{m}-\bar{\chi}^{2}+2 \lambda+2 \beta \mathcal{A}_{\mathcal{S}}^{m} \kappa_{\mathcal{S}}^{m}\right]-\vec{Y}_{\mathcal{S}}^{m} \cdot \vec{e}_{1}, \vec{\chi}_{\rho} \cdot \vec{\tau}^{m}\right)^{(h)}\right. \\
&-\left(\left[\pi\left[\alpha\left[\kappa_{\mathcal{S}}^{m}-\vec{\chi}^{2}+2 \lambda+2 \beta \mathcal{A}_{\mathcal{S}}^{m} \kappa_{\mathcal{S}}^{m}\right]-\kappa_{\mathcal{S}}^{m} \vec{Y}_{\mathcal{S}}^{m} \cdot \vec{\nu}^{m}\right]\left|\vec{X}_{\rho}^{m}\right|-\left(\vec{Y}_{\mathcal{S}}^{m}\right)_{\rho} \cdot \vec{\tau}^{m}, \vec{\chi} \cdot \vec{e}_{1}\right)^{(h)}\right. \\
&+\left(\vec{X}^{m} \cdot \vec{e}_{1} \kappa_{\mathcal{S}}^{m}\left(\vec{Y}_{\mathcal{S}}^{m}\right)^{\perp}, \vec{\chi}_{\rho}\right)^{(h)}-\sum_{p \in \partial_{2} I \cup \partial_{F} I}\left[\left[2 \pi \varsigma+\vec{m}^{m} \cdot \vec{Y}_{\mathcal{S}}^{m}\right]\left(\vec{\chi}^{\prime} \cdot \vec{e}_{1}\right)\right](p) \quad \forall \vec{\chi} \in \mathbb{X}^{h}, \\
& 2 \pi\left(\vec{X}^{m} \cdot \vec{e}_{1}\left(\alpha\left(\kappa_{\mathcal{S}}^{m+1}-\bar{\varkappa}\right)+\beta \mathcal{A}_{\mathcal{S}}^{m}\right), \chi\left|\vec{X}_{\rho}^{m}\right|\right)^{(h)}-\left(\left(\vec{X}^{m} \cdot \vec{e}_{1}\right) \vec{Y}_{\mathcal{S}}^{m+1}, \chi \vec{\nu}^{m}\left|\vec{X}_{\rho}^{m}\right|\right)^{(h)}=0 \quad \forall \chi \in W_{\left(\partial_{0}\right)}^{h}, \\
&\left(\vec{X}^{m} \cdot \vec{e}_{1} \kappa_{\mathcal{S}}^{m+1} \vec{\nu}^{m}, \vec{\eta}\left|\vec{X}_{\rho}^{m}\right|\right)^{(h)}+\left(\vec{e}_{1}, \vec{\eta}\left|\vec{X}_{\rho}^{m}\right|\right)+\left(\left(\vec{X}^{m} \cdot \vec{e}_{1}\right) \vec{X}_{\rho}^{m+1}, \vec{\eta}_{\rho}\left|\vec{X}_{\rho}^{m}\right|^{-1}\right)
\end{aligned}
$$




$$
=\sum_{p \in \partial_{C} I}\left[\left(\vec{X}^{m} \cdot \vec{e}_{1}\right) \vec{\zeta} \cdot \vec{\eta}\right](p)+\sum_{p \in \partial_{M} I}\left[\left(\vec{X}^{m} \cdot \vec{e}_{1}\right) \vec{m}^{m+1} \cdot \vec{\eta}\right](p) \quad \forall \vec{\eta} \in \underline{V}_{\partial_{0}}^{h}
$$

where

$$
\mathcal{A}_{\mathcal{S}}^{m}=2 \pi\left(\vec{X}^{m} \cdot \vec{e}_{1} \kappa_{\mathcal{S}}^{m},\left|\vec{X}_{\rho}^{m}\right|\right)^{(h)}-M_{0}
$$

We now state the analogues of Assumptions 5.1 and 5.2.

Assumption 5.8. Let $\operatorname{dim} \operatorname{span}\left\{\left(\left(\vec{X}^{m} \cdot \vec{e}_{1}\right) \vec{\nu}^{m}, \chi\left|\vec{X}_{\rho}^{m}\right|\right)^{(h)}: \chi \in W_{\left(\partial_{0}\right)}^{h}\right\}=2$.

Assumption 5.9. Let $\vec{X}^{m}$ satisfy Assumption 4.1 with $\vec{X}^{h}$ replaced by $\vec{X}^{m}$ and be such that the following holds. If $\vec{U} \in \mathbb{Y}^{h}(\overrightarrow{0})$ with

$$
\left(\left(\vec{X}^{m} \cdot \vec{e}_{1}\right) \vec{U}_{\rho}, \vec{\chi}_{\rho}\left|\vec{X}_{\rho}^{m}\right|^{-1}\right)=0 \quad \forall \vec{\chi} \in \mathbb{X}^{h} \quad \text { and } \quad\left(\left(\vec{X}^{m} \cdot \vec{e}_{1}\right) \vec{U}, \chi \vec{\nu}^{m}\left|\vec{X}_{\rho}^{m}\right|\right)^{(h)}=0 \quad \forall \chi \in W_{\left(\partial_{0}\right)}^{h},
$$

then $\vec{U}=\overrightarrow{0}$.

Lemma 5.10. Let Assumptions 5.1 and 5.8 hold. Moreover, if $\partial_{C} I \neq \emptyset$ then let Assumptions 5.9 hold. Let $\vec{X}^{m}, \vec{Y}_{\mathcal{S}}^{m} \in \underline{V}_{\partial_{0}}^{h}, \kappa_{\mathcal{S}}^{m} \in V^{h}, \vec{m}^{m} \in \mathbb{R}^{2}$ and $\alpha \in \mathbb{R}_{>0}, \bar{\varkappa}, \lambda, M_{0}, \alpha_{G} \in \mathbb{R}, \beta \in \mathbb{R}_{\geq 0}, \vec{\zeta}: \partial_{C} I \rightarrow \mathbb{S}^{1}$ be given. Then there exists a unique solution to $\left(\mathcal{P}_{\mathcal{S}}^{m}\right)^{(h)},(5.9)$.

Proof. As we have a linear system of equations, with the same number of equations as unknowns, existence follows from uniqueness. Hence we consider a solution to the homogeneous equivalent of (5.9), and need to show that this solution is in fact zero. In particular, let $\delta \vec{X} \in \mathbb{X}^{h}, \kappa_{\mathcal{S}} \in W_{\left(\partial_{0}\right)}^{h}, \vec{Y}_{\mathcal{S}} \in \underline{V}_{\partial_{0}}^{h}$, with $\pi^{h}\left[\left(\vec{X}^{m} \cdot \vec{e}_{1}\right) \vec{Y}_{\mathcal{S}}\right] \in$ $\mathbb{Y}^{h}(\overrightarrow{0})$, and $\vec{m} \in \mathbb{R}^{2}$ be such that

$$
\begin{aligned}
2 \pi\left(\left(\vec{X}^{m} \cdot \vec{e}_{1}\right) \underline{\underline{Q}}^{m} \frac{\delta \vec{X}}{\Delta t_{m}}, \vec{\chi}\left|\vec{X}_{\rho}^{m}\right|\right)^{h}-\left(\left(\vec{X}^{m} \cdot \vec{e}_{1}\right)\left(\vec{Y}_{\mathcal{S}}\right)_{\rho}, \vec{\chi}_{\rho}\left|\vec{X}_{\rho}^{m}\right|^{-1}\right)=0 & \forall \vec{\chi} \in \mathbb{X}^{h}, \\
2 \pi \alpha\left(\vec{X}^{m} \cdot \vec{e}_{1} \kappa_{\mathcal{S}}, \chi\left|\vec{X}_{\rho}^{m}\right|\right)^{(h)}-\left(\left(\vec{X}^{m} \cdot \vec{e}_{1}\right) \vec{Y}_{\mathcal{S}}, \chi \vec{\nu}^{m}\left|\vec{X}_{\rho}^{m}\right|\right)^{(h)}=0 & \forall \chi \in W_{\left(\partial_{0}\right.}^{h} \\
\left(\vec{X}^{m} \cdot \vec{e}_{1} \kappa_{\mathcal{S}} \vec{\nu}^{m}, \vec{\eta}\left|\vec{X}_{\rho}^{m}\right|\right)^{(h)}+\left(\left(\vec{X}^{m} \cdot \vec{e}_{1}\right)(\delta \vec{X})_{\rho}, \vec{\eta}_{\rho}\left|\vec{X}_{\rho}^{m}\right|^{-1}\right)=\sum_{p \in \partial_{M} I}\left[\left(\vec{X}^{m} \cdot \vec{e}_{1}\right) \vec{m} \cdot \vec{\eta}\right](p) & \forall \vec{\eta} \in \underline{V}_{\partial_{0}}^{h} .
\end{aligned}
$$

Choosing $\vec{\chi}=\delta \vec{X}$ in (5.10a), $\chi=\kappa_{\mathcal{S}}$ in $(5.10 \mathrm{~b})$ and $\vec{\eta}=\vec{Y}_{\mathcal{S}}$ in $(5.10 \mathrm{c})$ yields, on noting $\pi^{h}\left[\left(\vec{X}^{m} \cdot \vec{e}_{1}\right) \vec{Y}_{\mathcal{S}}\right] \in$ $\mathbb{Y}^{h}(\overrightarrow{0})$, that

$$
2 \pi\left(\left.\vec{X}^{m} \cdot \vec{e}_{1} \underline{\underline{Q}}^{m} \delta \vec{X}\right|^{2},\left|\vec{X}_{\rho}^{m}\right|\right)^{h}+2 \pi \alpha \Delta t_{m}\left(\vec{X}^{m} \cdot \vec{e}_{1}\left(\kappa_{\mathcal{S}}\right)^{2},\left|\vec{X}_{\rho}^{m}\right|\right)^{(h)}=0 .
$$

It follows from (5.11) and $\kappa_{\mathcal{S}} \in W_{\left(\partial_{0}\right)}^{h}$ that $\kappa_{\mathcal{S}}=0$. Similarly, it follows from (5.11) that $\left[\delta \vec{X} \cdot \vec{v}^{m}\right]\left(q_{j}\right)=0$, $j=1, \ldots, J-1$, and, on recalling (4.7), that $\delta \vec{X}=\overrightarrow{0}$ on $\partial I \backslash \partial_{0} I$. Then choosing $\vec{\eta}=\delta \vec{X} \in \mathbb{X}^{h} \subset \underline{V}_{\partial_{0}}^{h}$ in (5.10c) yields, on recalling (2.37) and $\delta \vec{X}=\overrightarrow{0}$ on $\partial I \backslash \partial_{0} I$, that

$$
\left(\vec{X}^{m} \cdot \vec{e}_{1}\left|(\delta \vec{X})_{\rho}\right|^{2},\left|\vec{X}_{\rho}^{m}\right|^{-1}\right)=\sum_{p \in \partial_{M} I}\left[\left(\vec{X}^{m} \cdot \vec{e}_{1}\right) \vec{m} \cdot \delta \vec{X}\right](p)=0 .
$$


It follows from (5.12) that $\delta \vec{X}$ is constant in $\bar{I}$. Together with $\left[\delta \vec{X} \cdot \vec{v}^{m}\right]\left(q_{j}\right)=0, j=1, \ldots, J-1$, and Assumption 5.1 we obtain that $\delta \vec{X}=\overrightarrow{0}$. Then (5.10c) implies that $\vec{m}=\overrightarrow{0}$.

If $\partial_{C} I=\emptyset$, then we can choose $\vec{\chi}=\vec{Y}_{\mathcal{S}} \in \mathbb{Y}^{h}(\overrightarrow{0}) \subset \mathbb{X}^{h}$ in $(5.10 \mathrm{a})$, recall (3.7), to obtain that $\vec{Y}_{\mathcal{S}}$ is constant in $\bar{I}$. Then (5.10b), together with Assumption 5.8, gives that $\vec{Y}_{\mathcal{S}}=\overrightarrow{0}$. If $\partial_{C} I \neq \emptyset$, on the other hand, then Assumption 5.9 directly gives that $\vec{Y}_{\mathcal{S}}=\overrightarrow{0}$. Hence we have shown the existence of a unique solution to $\left(\mathcal{P}_{\mathcal{S}}^{m}\right)^{h}$.

Remark 5.11. Similarly to Remark 5.6, in practice the system (5.9) is easiest solved by first eliminating $\vec{m}^{m+1}$. This can be achieved by replacing the test space $\underline{V}_{\partial_{0}}^{h}$ in $(5.9 \mathrm{c})$ with $\mathbb{Y}^{h}(\overrightarrow{0})$. Having computed $\left(\delta \vec{X}^{m+1}, \kappa_{\mathcal{S}}^{m+1}, \vec{Y}_{\mathcal{S}}^{m+1}\right)$, the values $\vec{m}^{m+1}$ can then be obtained from (5.9c). For example, if $q_{0} \in \partial_{M} I$ then we have that

$$
\begin{aligned}
\left(\vec{X}^{m}\left(q_{0}\right) \cdot \vec{e}_{1}\right) \vec{m}^{m+1}\left(q_{0}\right)= & \left(\vec{X}^{m} \cdot \vec{e}_{1} \kappa_{\mathcal{S}}^{m+1}, \chi_{0}\left|\vec{X}_{\rho}^{m}\right|\right)^{(h)} \vec{\nu}^{m}\left(q_{0}\right)+\left(1, \chi_{0}\left|\vec{X}_{\rho}^{m}\right|\right) \vec{e}_{1} \\
& +\left(\vec{X}^{m} \cdot \vec{e}_{1},\left(\chi_{0}\right)_{\rho}\left|\vec{X}_{\rho}^{m}\right|^{-1}\right) \vec{X}_{\rho}^{m+1}\left(q_{0}\right)
\end{aligned}
$$

\section{Numerical RESUlts}

On recalling $(4.13),(4.15)$ and $(4.30)$, as the fully discrete energy for the two schemes $\left(\mathcal{P}^{m}\right)^{h}$ and $\left(\mathcal{P}_{\mathcal{S}}^{m}\right)^{(h)}$ we consider, respectively,

$$
\begin{aligned}
\widehat{E}^{m+1}= & \pi\left(\alpha\left[\mathfrak{K}^{h}\left(\vec{X}^{m}, \kappa^{m+1}\right)-\bar{\varkappa}^{2}+2 \lambda, \vec{X}^{m} \cdot \vec{e}_{1}\left|\vec{X}_{\rho}^{m}\right|\right)^{h}+\frac{\beta}{2}\left[2 \pi\left(\vec{X}^{m} \cdot \vec{e}_{1} \kappa^{m+1}-\vec{\nu}^{m} \cdot \vec{e}_{1},\left|\vec{X}_{\rho}^{m}\right|\right)^{h}-M_{0}\right]^{2}\right. \\
& -2 \pi \alpha_{G} \sum_{p \in \partial_{M} I} \vec{m}^{m+1}(p) \cdot \vec{e}_{1}+2 \pi \varsigma \sum_{p \in \partial_{2} I \cup \partial_{F} I} \vec{X}^{m+1}(p) \cdot \vec{e}_{1} \\
\widehat{E}_{\mathcal{S}}^{m+1}= & \pi\left(\alpha\left[\kappa_{\mathcal{S}}^{m+1}-\bar{\varkappa}^{2}+2 \lambda, \vec{X}^{m} \cdot \vec{e}_{1}\left|\vec{X}_{\rho}^{m}\right|\right)^{(h)}+\frac{\beta}{2}\left[2 \pi\left(\vec{X}^{m} \cdot \vec{e}_{1} \kappa_{\mathcal{S}}^{m+1},\left|\vec{X}_{\rho}^{m}\right|\right)^{(h)}-M_{0}\right]^{2}\right. \\
& -2 \pi \alpha_{G} \sum_{p \in \partial_{M} I} \vec{m}^{m+1}(p) \cdot \vec{e}_{1}+2 \pi \varsigma \sum_{p \in \partial_{2} I \cup \partial_{F} I} \vec{X}^{m+1}(p) \cdot \vec{e}_{1} .
\end{aligned}
$$

Given $\Gamma^{0}=\vec{X}^{0}(I)$, we define the following initial data. First, we let $\vec{\mu}^{0}$ be the true conormal to $\Gamma^{0}$, i.e. $\vec{\mu}^{0}=(-1)^{p+1} \vec{\tau}^{0}(p)$ for $p \in \partial I \backslash \partial_{0} I$, recall $(2.20)$, and then set $\vec{m}^{0}=\vec{\mu}^{0}$ on $\partial_{M} I$. Next, on recalling (4.10), we let $\vec{\kappa}^{0} \in \underline{V}_{\partial_{0}}^{h}$ be such that

$$
\left(\vec{\kappa}^{0}, \vec{\eta}\left|\vec{X}_{\rho}^{0}\right|\right)^{h}+\left(\vec{\tau}^{0}, \vec{\eta}_{\rho}\right)=\sum_{p \in \partial I \backslash \partial_{0} I}\left[\vec{\mu}^{0} \cdot \vec{\eta}\right](p) \quad \forall \vec{\eta} \in \underline{V}_{\partial_{0}}^{h},
$$

and then define $\kappa^{0}=\pi_{\partial_{0}}^{h}\left[\vec{\kappa}^{0} \cdot \vec{v}^{0}\right] \in W_{\partial_{0}}^{h}$. Moreover, we let $\vec{Y}_{\star}^{0} \in \underline{V}^{h}$ be such that

$$
\vec{Y}_{\star}^{0}=2 \pi \vec{\pi}^{h}\left[\left|\vec{\omega}^{0}\right|^{-1} \vec{X}^{0} \cdot \vec{e}_{1}\left(\alpha\left[\mathfrak{K}^{h}\left(\vec{X}^{0}, \kappa^{0}\right)-\bar{\varkappa}\right]+\beta \mathcal{A}^{0}\right) \vec{v}^{0}\right],
$$

recall (4.15), (4.5), (4.6) and (5.1d), and then define $\vec{Y}^{0} \in \mathbb{Y}^{h}\left(2 \pi \alpha_{G} \vec{e}_{1}\right)$ via

$$
\vec{Y}^{0}\left(q_{j}\right)= \begin{cases}\left(\vec{Y}_{\star}^{0}\left(q_{j}\right) \cdot \vec{e}_{2}\right) \vec{e}_{2} & q_{j} \in \partial_{0} I, \\ 2 \pi \alpha_{G} \vec{e}_{1} & q_{j} \in \partial_{M} I, \\ \vec{Y}_{\star}^{0}\left(q_{j}\right) & q_{j} \in \bar{I} \backslash\left(\partial_{0} I \cup \partial_{M} I\right) .\end{cases}
$$



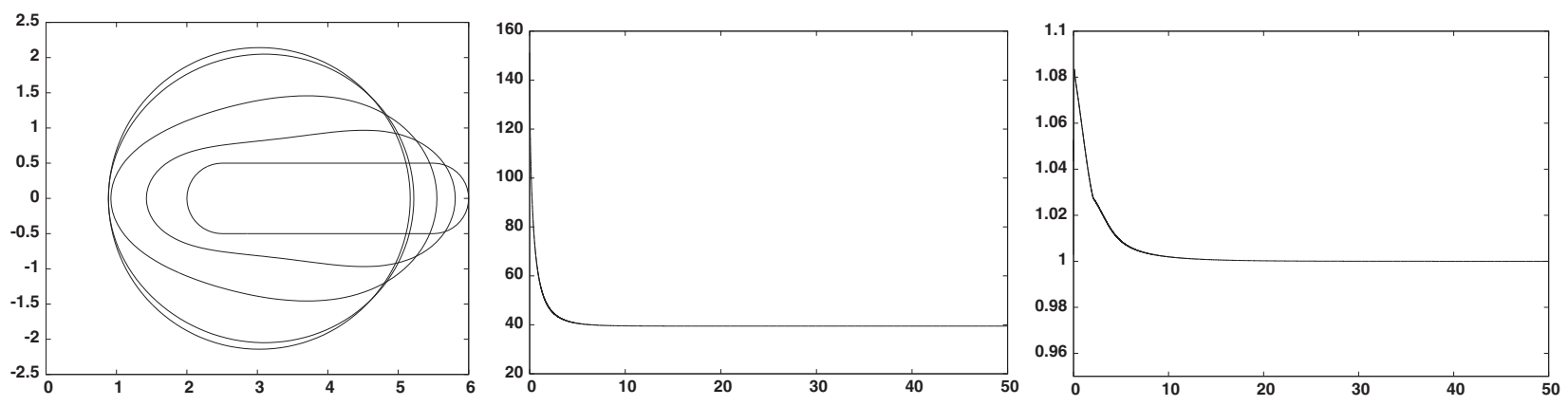

Figure 2. $\left(\mathcal{P}^{m}\right)^{h}$ Willmore flow for a torus. Solution at times $t=0,0.5,2,10,50$. Below a plot of the discrete energy (6.1a) and of the ratio (6.2).

In addition, we set $\kappa_{\mathcal{S}}^{0}=\mathfrak{K}^{h}\left(\vec{X}^{0}, \kappa^{0}\right) \in V^{h}$, and let $\vec{Y}_{\mathcal{S}, \star}^{0} \in \underline{V}^{h}$ be such that

$$
\vec{Y}_{\mathcal{S}, \star}^{0}=2 \pi \vec{\pi}^{h}\left[\left|\vec{\omega}^{0}\right|^{-1}\left(\alpha\left[\kappa_{\mathcal{S}}^{0}-\bar{\varkappa}\right]+\beta \mathcal{A}_{\mathcal{S}}^{0}\right) \vec{v}^{0}\right]
$$

recall (4.41b), (4.6) and (5.9d). Then we define $\vec{Y}_{\mathcal{S}}^{0} \in \underline{V}_{\partial_{0}}^{h}$, with $\vec{\pi}^{h}\left[\left(\vec{X}^{0} \cdot \vec{e}_{1}\right) \vec{Y}_{\mathcal{S}}^{0}\right] \in \mathbb{Y}^{h}\left(2 \pi \alpha_{G} \vec{e}_{1}\right)$, via

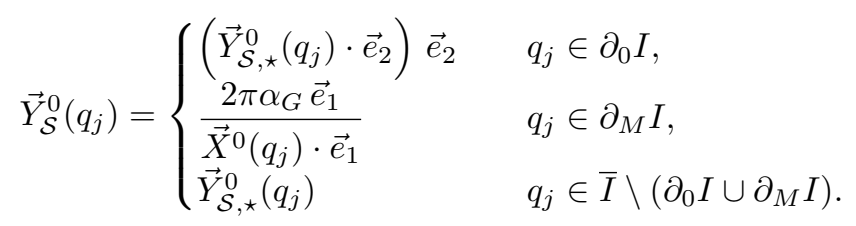

Unless otherwise stated we use $\alpha=1, \bar{\varkappa}=\lambda=\beta=\alpha_{G}=\varsigma=0$ and employ uniform time steps, $\Delta t_{m}=\Delta t$, $m=0, \ldots, M-1$.

\subsection{Surfaces without boundary}

\subsubsection{Genus 1 surfaces}

In this subsection, we consider genus 1 surfaces without boundary, so that $\partial I=\emptyset$.

Starting with an elongated cigar-like shape for $\Gamma^{0}$, we observe the evolution shown in Figure 2 for the scheme $\left(\mathcal{P}^{m}\right)^{h},(5.1)$. The discretization parameters are $J=128$ and $\Delta t=10^{-4}$. The observed final radius is 2.14, with the centre at $(3.03,0)$. Hence $R / r=3.03 / 2.14=1.414 \approx \sqrt{2}$. Here we recall that the ratio $\sqrt{2}$ characterizes the Clifford torus, the known minimizer of the Willmore energy (2.13), with $\bar{\varkappa}=0$ and $\alpha=1$, among all genus 1 surfaces, see [28], with Willmore energy equal to $4 \pi^{2}=39.478$. We note that, as expected, the energy (6.1a) is monotonically decreasing, while the ratio

$$
r^{m}=\frac{\max _{j=1, \ldots, J}\left|\vec{X}^{m}\left(q_{j}\right)-\vec{X}^{m}\left(q_{j-1}\right)\right|}{\min _{j=1, \ldots, J}\left|\vec{X}^{m}\left(q_{j}\right)-\vec{X}^{m}\left(q_{j-1}\right)\right|}
$$

approaches one as time increases.

When we repeat the simulation for the two schemes $\left(\mathcal{P}_{\mathcal{S}}^{m}\right)^{(h)}$, we note markedly different tangential motions. For the scheme with mass lumping throughout, $\left(\mathcal{P}_{\mathcal{S}}^{m}\right)^{h}$, the vertices coalesce on the left side of the curve and eventually the algorithm breaks down. For the scheme $\left(\mathcal{P}_{\mathcal{S}}^{m}\right)$, on the other hand, the density of vertices is higher on the right side of the circular shape, with the ratio (6.2) smaller than 2. We demonstrate this in Figure 3, where we also show an evolution of the ratio $(6.2)$ for the scheme $\left(\mathcal{P}_{\mathcal{S}}^{m}\right)$ over time. 

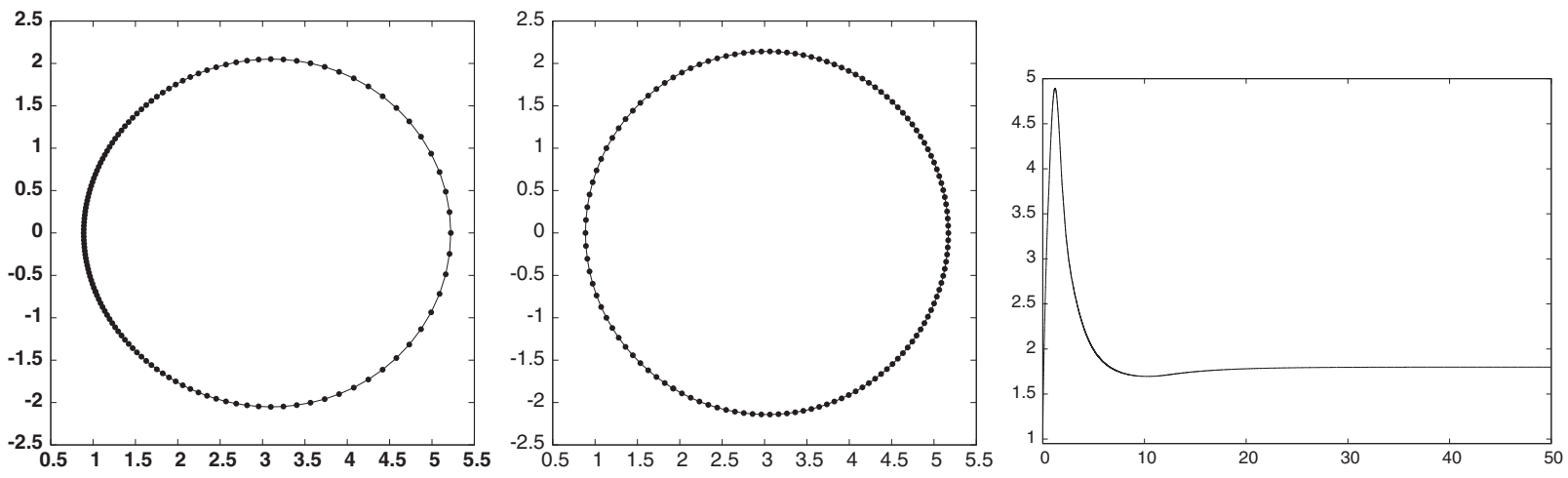

Figure 3. $\left(\mathcal{P}_{\mathcal{S}}^{m}\right)^{(h)}$ Same evolution as in Figure 2. Left: $\Gamma^{m}$ at time $t=10$ for $\left(\mathcal{P}_{\mathcal{S}}^{m}\right)^{h}$, middle: $\Gamma^{m}$ at time $t=50$ for $\left(\mathcal{P}_{\mathcal{S}}^{m}\right)$, right: the ratio $(6.2)$ over time for $\left(\mathcal{P}_{\mathcal{S}}^{m}\right)$.

Because of the bad behaviour of the scheme $\left(\mathcal{P}_{\mathcal{S}}^{m}\right)^{h}$ in practice, we will discard that scheme from now on. We will mainly concentrate on the scheme $\left(\mathcal{P}^{m}\right)^{h}$, which in practice leads to nearly equidistributed polygonal curves, and at times compare it to the scheme $\left(\mathcal{P}_{\mathcal{S}}^{m}\right)$.

\subsubsection{Genus 0 surfaces}

In this subsection, we consider genus 0 surfaces without boundary, so that $\partial_{0} I=\{0,1\}$. We will parameterize $\Gamma$ clockwise, so that $\vec{\nu}$ induces the outer normal $\vec{n}_{\mathcal{S}}$ on $\mathcal{S}$, recall (2.3) and (2.4).

We begin with a convergence experiment. To this end, we note that a sphere of radius $R(t)$, where $R(t)$ satisfies

$$
R^{\prime}(t)=-\frac{\bar{\varkappa}}{R(t)}\left(\frac{2}{R(t)}+\bar{\varkappa}\right), \quad R(0)=R_{0} \in \mathbb{R}_{>0},
$$

is a solution to (2.14). The nonlinear $\operatorname{ODE}(6.3)$, in the case $\bar{\varkappa} \neq 0$, is solved by $R(t)=z(t)-\frac{2}{\bar{\varkappa}}$, where $z(t)$ is such that $\frac{1}{2}\left(z^{2}(t)-z_{0}^{2}\right)-\frac{4}{\bar{\varkappa}}\left(z(t)-z_{0}\right)+\frac{4}{\bar{\varkappa}^{2}} \ln \frac{z(t)}{z_{0}}+\bar{\varkappa}^{2} t=0$, with $z_{0}=R_{0}+\frac{2}{\bar{\varkappa}}$. We use the solution to (6.3), with $\bar{\varkappa}=-1$, and a sequence of approximations for the unit sphere $\left(R_{0}=1\right)$ to compute the error $\left\|\Gamma-\Gamma^{h}\right\|_{L^{\infty}}=\max _{m=1, \ldots, M} \max _{j=0, \ldots, J}|| \vec{X}^{m}\left(q_{j}\right)\left|-R\left(t_{m}\right)\right|$ over the time interval $[0,1]$ between the true solution and the discrete solutions for the schemes $\left(\mathcal{P}^{m}\right)^{h}$ and $\left(\mathcal{P}_{\mathcal{S}}^{m}\right)$. In particular, we choose $\vec{X}^{0} \in \underline{V}_{\partial_{0}}^{h}$ with

$$
\vec{X}^{0}\left(q_{j}\right)=\left(\begin{array}{c}
\cos \left[\left(\frac{1}{2}-q_{j}\right) \pi+0.1 \cos \left(\left(\frac{1}{2}-q_{j}\right) \pi\right)\right] \\
\sin \left[\left(\frac{1}{2}-q_{j}\right) \pi+0.1 \cos \left(\left(\frac{1}{2}-q_{j}\right) \pi\right)\right]
\end{array}\right), \quad j=0, \ldots, J,
$$

recall (4.1), to ensure an initially non-uniform distribution of vertices. We also define the error

$$
\left\|\Gamma-\Gamma^{h}\right\|_{L^{\infty}\left(L^{2}\right)}=\max _{m=1, \ldots, M}\left[\left(|| \vec{X}^{m}\left|-R\left(t_{m}\right)\right|^{2},\left|\vec{X}_{\rho}^{m}\right|\right)^{h}\right]^{\frac{1}{2}} .
$$

Here we used the time step size $\Delta t=0.1 h_{\Gamma^{0}}^{2}$, where $h_{\Gamma^{0}}$ is the maximal edge length of $\Gamma^{0}$. The computed errors, together with their experimental order of convergence (EOC), are reported in Tables 2 and 3. We remark that repeating these simulations for the scheme $\left(\mathcal{P}_{\mathcal{S}}^{m}\right)^{h}$ failed, because of tangential motion leading to vertices in the left halfplane.

We remark that for the experiments in Table 2, the ratio (6.2), which at time $t=0$ starts off at about $r^{0}=1.22$, always decreases monotonically and approaches the value 1 , so that the final semicircle is nearly equidistributed. For the experiments in Table 3, however, this is not the case, and the distribution of vertices 
TABLE 2. Errors for the convergence test for the scheme $\left(\mathcal{P}^{m}\right)^{h}$ with $\bar{\varkappa}=-1$. We also show the ratio (6.2) at time $t=1$.

\begin{tabular}{lllllll}
\hline \hline$J$ & $h_{\Gamma^{0}}$ & $\left\|\Gamma-\Gamma^{h}\right\|_{L^{\infty}}$ & EOC & $\left\|\Gamma-\Gamma^{h}\right\|_{L^{\infty}\left(L^{2}\right)}$ & EOC & $r^{M}$ \\
\hline 32 & $1.0792 \mathrm{e}-01$ & $1.3951 \mathrm{e}-02$ & - & $7.3622 \mathrm{e}-03$ & - & 1.06 \\
64 & $5.3988 \mathrm{e}-02$ & $4.1092 \mathrm{e}-03$ & 1.76 & $1.8232 \mathrm{e}-03$ & 2.02 & 1.06 \\
128 & $2.6997 \mathrm{e}-02$ & $1.1867 \mathrm{e}-03$ & 1.79 & $4.5434 \mathrm{e}-04$ & 2.00 & 1.06 \\
256 & $1.3499 \mathrm{e}-02$ & $3.3690 \mathrm{e}-04$ & 1.82 & $1.1347 \mathrm{e}-04$ & 2.00 & 1.06 \\
512 & $6.7495 \mathrm{e}-03$ & $9.4318 \mathrm{e}-05$ & 1.84 & $2.8358 \mathrm{e}-05$ & 2.00 & 1.06 \\
\hline
\end{tabular}

TABLE 3. Errors for the convergence test for the scheme $\left(\mathcal{P}_{\mathcal{S}}^{m}\right)$ with $\bar{\varkappa}=-1$. We also show the ratio (6.2) at time $t=1$.

\begin{tabular}{lllllll}
\hline \hline$J$ & $h_{\Gamma^{0}}$ & $\left\|\Gamma-\Gamma^{h}\right\|_{L^{\infty}}$ & EOC & $\left\|\Gamma-\Gamma^{h}\right\|_{L^{\infty}\left(L^{2}\right)}$ & EOC & $r^{M}$ \\
\hline 32 & $1.0792 \mathrm{e}-01$ & $1.5595 \mathrm{e}-02$ & - & $9.8711 \mathrm{e}-03$ & - & 2.79 \\
64 & $5.3988 \mathrm{e}-02$ & $5.5381 \mathrm{e}-03$ & 1.49 & $2.7237 \mathrm{e}-03$ & 1.86 & 3.32 \\
128 & $2.6997 \mathrm{e}-02$ & $1.9703 \mathrm{e}-03$ & 1.49 & $7.5278 \mathrm{e}-04$ & 1.86 & 3.96 \\
256 & $1.3499 \mathrm{e}-02$ & $7.0062 \mathrm{e}-04$ & 1.49 & $2.0783 \mathrm{e}-04$ & 1.86 & 4.72 \\
512 & $6.7495 \mathrm{e}-03$ & $2.4888 \mathrm{e}-04$ & 1.49 & $5.7280 \mathrm{e}-05$ & 1.86 & 5.61 \\
\hline
\end{tabular}
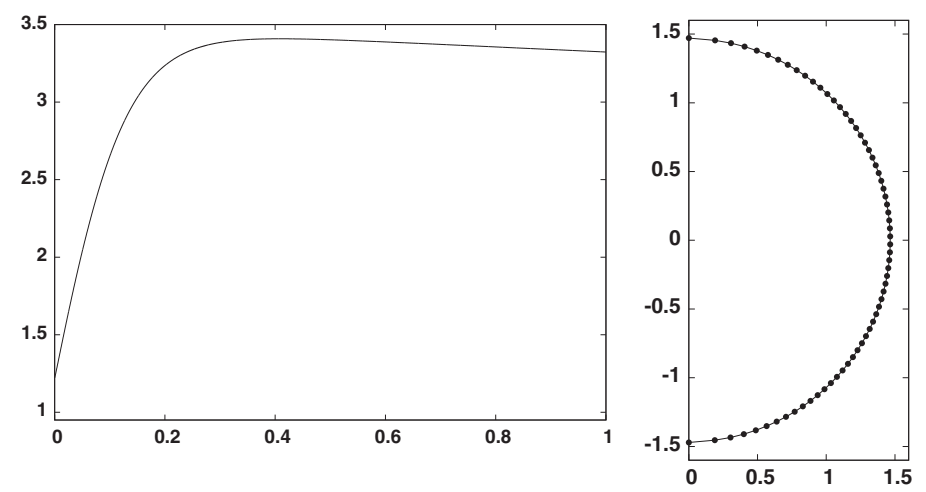

Figure 4. $\left(\mathcal{P}_{\mathcal{S}}^{m}\right)$ Generalized Willmore flow with $\bar{\varkappa}=-1$ for a sphere. A plot of (6.2) over time and a plot of the solution at time $t=1$ for the run with $J=64$ from Table 3 .

remains very nonuniform. In each case, the longest elements are the two elements touching the $\vec{e}_{2}$-axis, while the shortest elements are found far away from the axis. As an example, we show the plot of (6.2) over time, as well as the distribution of vertices at time $t=1$ for the run with $J=64$ in Figure 4, and these are generic for the behaviour for every value of $J$.

We conjecture that these long elements at the boundary are the reason for the suboptimal convergence rates seen in Table 3. We note that in $L^{\infty}\left(L^{1}\right)$ the convergence order is quadratic for the scheme $\left(\mathcal{P}_{\mathcal{S}}^{m}\right)$, although we do not display the precise numbers here.

Next we consider a numerical experiment for Helfrich flow, i.e. surface area and volume preserving Willmore flow, for a flat disc. The dimensions of the initial disc are chosen as $5 \times 1 \times 5$, so that the flow evolves towards a sharp that resembles a human red blood cell, see Figure 5. The discretization parameters for the two schemes are $J=128, \Delta t=10^{-4}$ and $T=0.2$. 

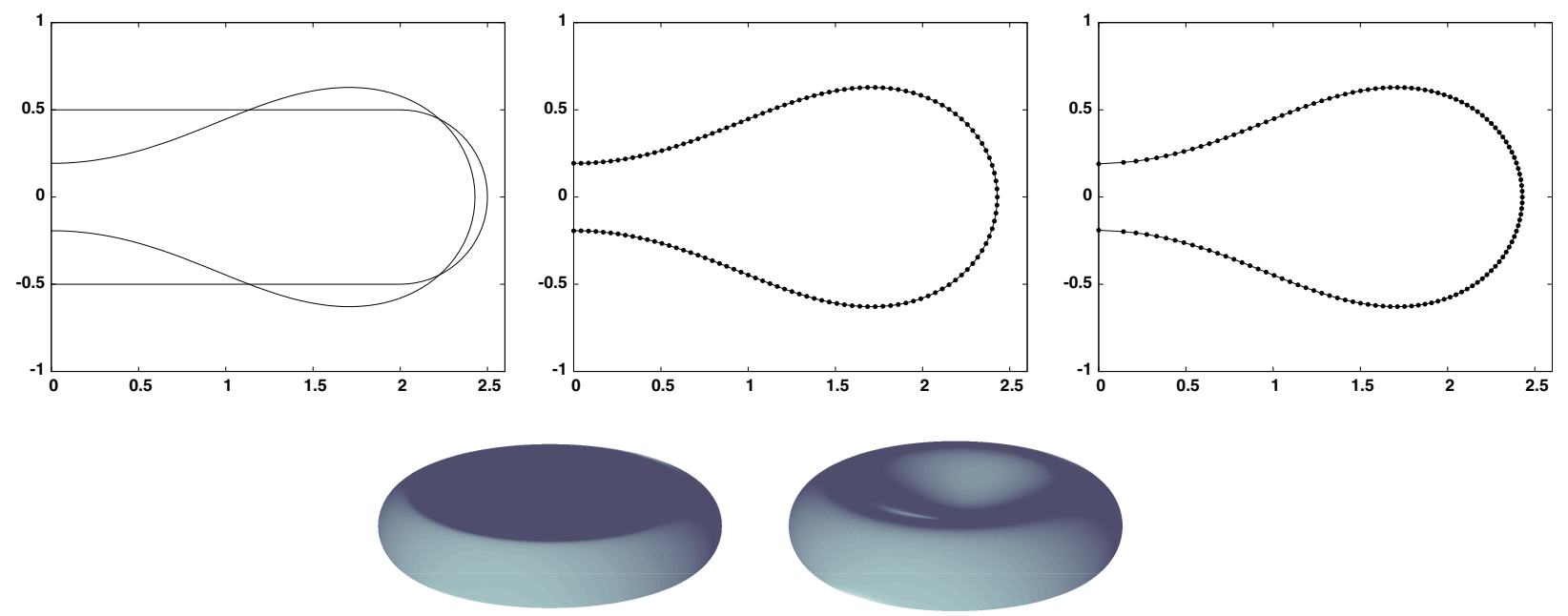

Figure 5. $\left(\mathcal{P}^{m}\right)^{h}$ Helfrich flow for a flat disc of dimension $5 \times 1 \times 5$. Solution at times $t=0,0.2$, and separately at time $t=0.2$. On the right we show the final distribution of vertices for the scheme $\left(\mathcal{P}_{\mathcal{S}}^{m}\right)$. Below we visualize the axisymmetric surfaces generated by $\Gamma^{m}$ at times $t=0$ and $t=0.2$.

\subsection{Surfaces with boundary}

In the case of clamped boundary conditions, recall (2.32), we define $\vec{\zeta}(p)$, for $p \in \partial_{C} I$, via $\vec{\zeta}(p)=$ $(\sin \vartheta(p), \cos \vartheta(p))^{T}$, where $\vartheta(p) \in \mathbb{R}, p \in \partial_{C} I$, denote the prescribed contact angle with the $x_{1}$-axis.

We recall from Lemmas 5.4 and 5.10 that we can prove existence of a unique solution to the linear systems arising at each time level in the presence of clamped boundary conditions only if Assumption 5.2 holds. This assumption is violated if $\Gamma^{m}$ is a straight line, and so the linear systems are indeed singular. In all other cases, however, the linear systems for the simulations we present in the following are nonsingular, and so we can find their unique solutions.

\subsubsection{Surfaces with one connected boundary component}

In this subsection, we consider the situation $\partial_{0} I=\{0\}$ and $\partial I=\{0,1\}$. On recalling (2.3) this means that the normal $\vec{\nu}(0, t)$ will point upwards.

We show two experiments for Navier boundary conditions in Figure 6, where we observe that depending on the sign of the spontaneous curvature, the sphere-like cap is either convex or concave. Our numerical results are in agreement with Figures 9 and 10 in [6]. As the discretization parameters we choose $J=64$ and $\Delta t=10^{-4}$.

The same simulations as in Figures 6, but for clamped boundary conditions are shown in Figure 7 .

\subsubsection{Surfaces with two connected boundary components}

In this subsection, we consider the case $\partial_{0} I=\emptyset$ and $\partial I=\{0,1\}$. We will always parameterize $\Gamma(0)$ from left to right, so that the normal $\vec{\nu}$ for a straight line points upwards. When the curve $\Gamma(0)$ is vertical, we parameterize it from top to bottom so that the normal $\vec{\nu}$ points to the right. Throughout this subsection we choose the discretization parameters $J=64$ and $\Delta t=10^{-4}$.

Navier boundary conditions for an open cylinder, when $\Gamma(0)$ is a straight vertical line, are shown in Figure 8. Here we note that for $\bar{\varkappa}=-1$ the evolution is stationary, which is in contrast to Figure 14, below.

In order to see the influence of a negative spontaneous curvature on the evolution, we start two simulations for Navier conditions for a cut cylinder with a dumbbell shape, see Figure 9. We observe that the dumbbell 

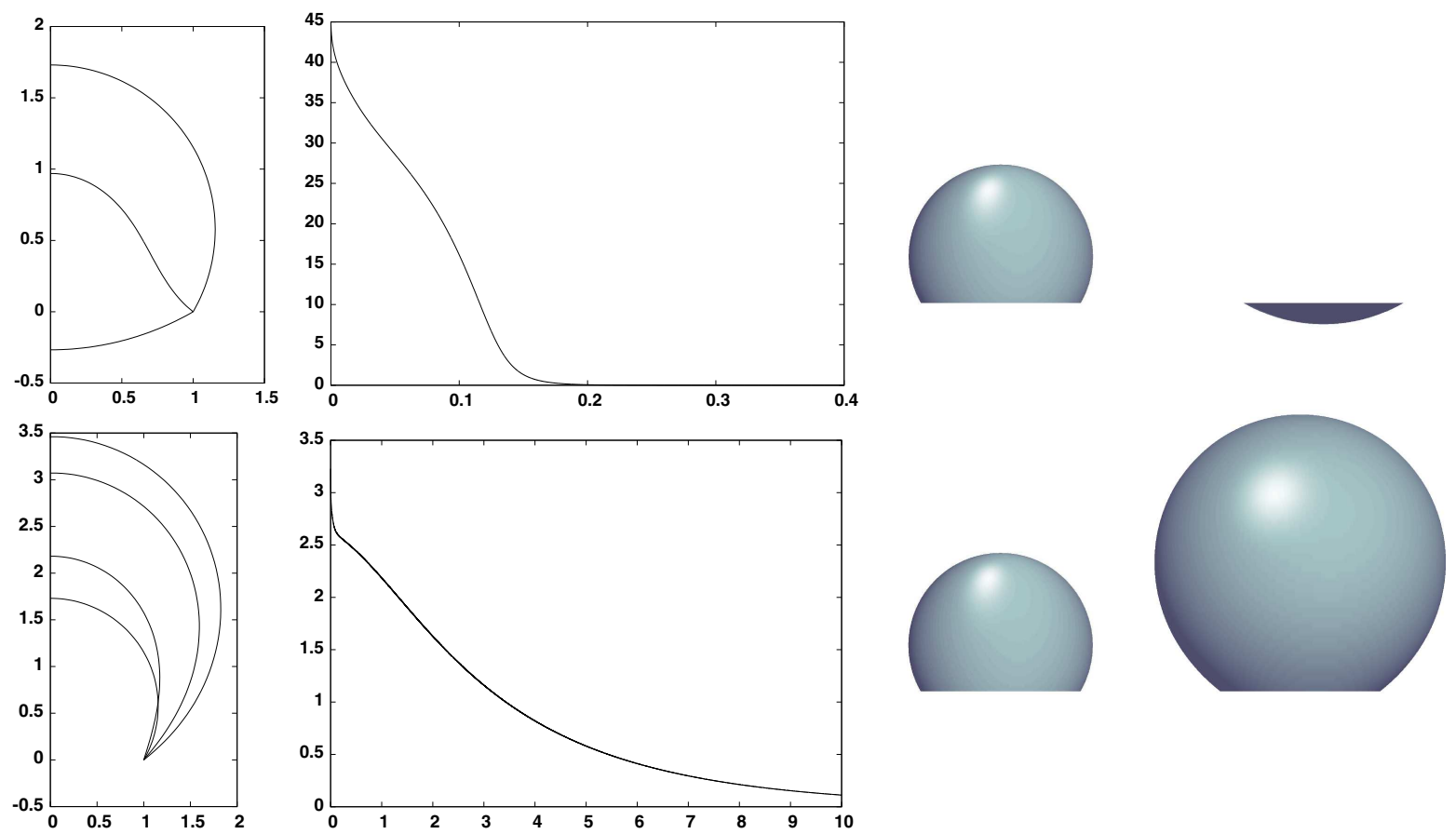

FiguRE 6. $\left(\mathcal{P}^{m}\right)^{h}$ Generalized Willmore flow with Navier boundary conditions for a spherelike cap with $\bar{\varkappa}=1$, top, and $\bar{\varkappa}=-1$, bottom. Solution at times $t=0,0.1,0.4$ (top) and $t=0,1,5,10$ (bottom), as well as a plot of the discrete energy (6.1a) over time. We also show the axisymmetric surfaces generated by $\Gamma^{0}$ and $\Gamma^{M}$.
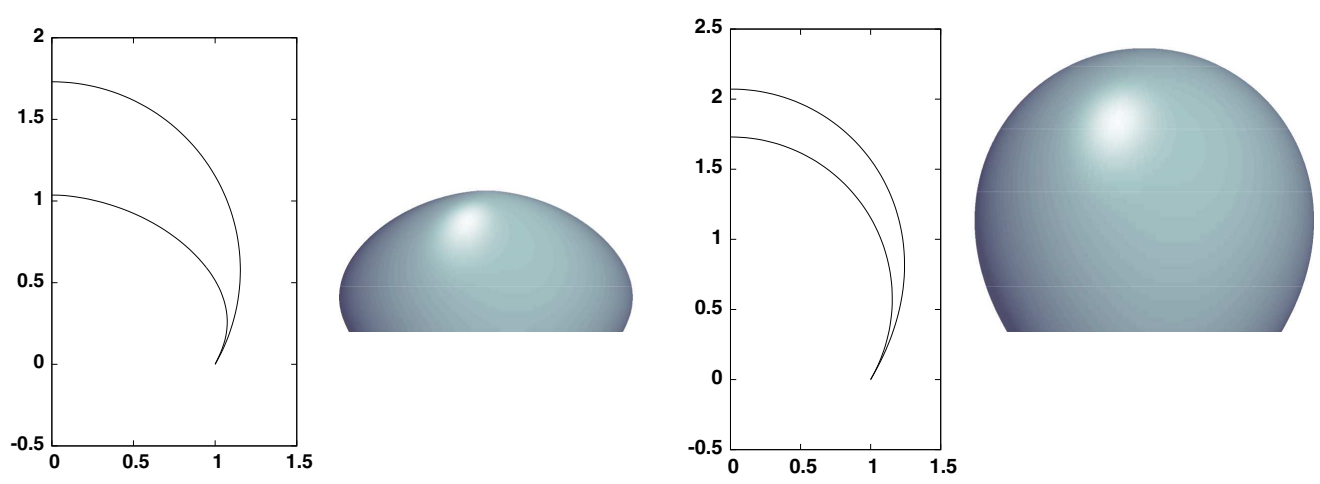

FIGURE 7. $\left(\mathcal{P}^{m}\right)^{h}$ Generalized Willmore flow with clamped boundary conditions with conormal angle $\vartheta(1)=\frac{7}{6} \pi=210^{\circ}$ for a sphere-like cap with $\bar{\varkappa}=1$, left, and $\bar{\varkappa}=-1$, right. The solutions are shown at times $t=0,0.5$, and we also visualize the axisymmetric surfaces generated by $\Gamma^{M}$. 

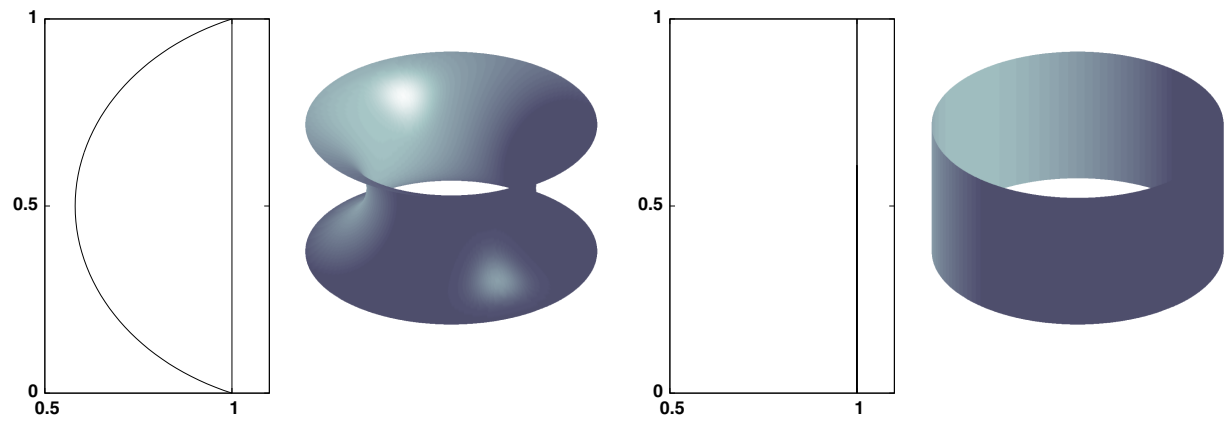

FigURE 8. $\left(\mathcal{P}^{m}\right)^{h}$ Generalized Willmore flow with Navier boundary conditions for an open cylinder. Left with $\bar{\varkappa}=1$, right with $\bar{\varkappa}=-1$. The solutions are shown at times $t=0,0.5$, and we also visualize the axisymmetric surfaces generated by $\Gamma^{M}$.
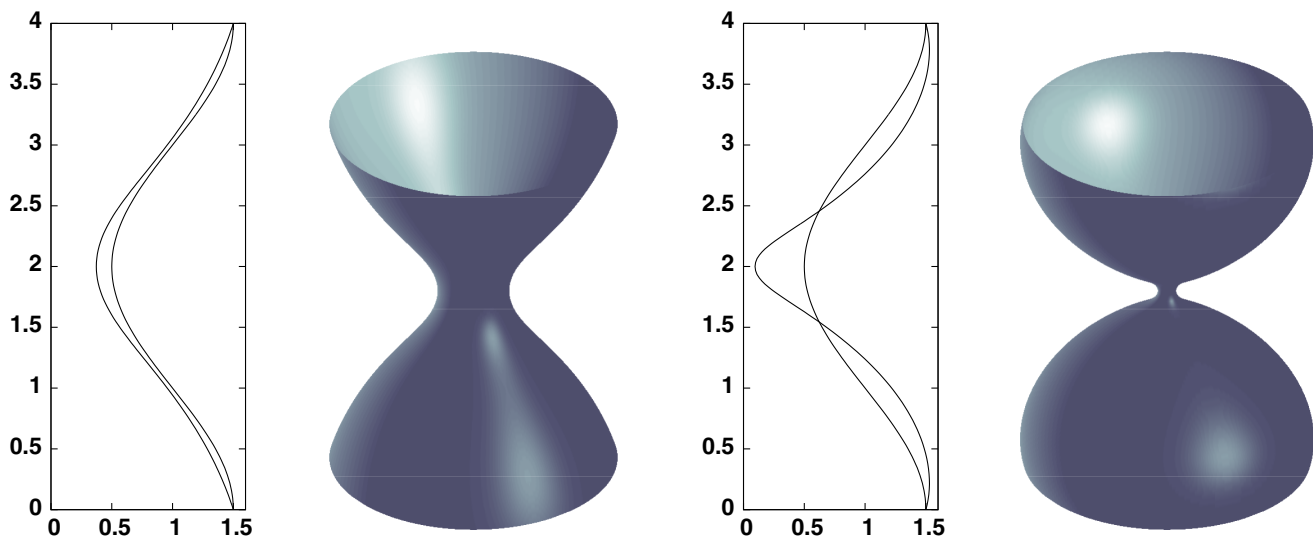

FiguRE 9. $\left(\mathcal{P}^{m}\right)^{h}$ Generalized Willmore flow with Navier boundary conditions for a dumbbelllike open cylinder shape. Left with $\bar{\varkappa}=-1$, right with $\bar{\varkappa}=-2$. The solutions are shown at times $t=0,1$, and we also visualize the axisymmetric surfaces generated by $\Gamma^{M}$.

becomes more and more pronounced, the smaller we choose $\bar{\varkappa}$. For $\bar{\varkappa}=-2$ the evolution nearly leads to a pinch-off. Choosing $\bar{\varkappa}=-3$ does indeed lead to pinch-off, which we do not show in Figure 9.

Two simulations for the same initial data, but now with $\partial_{F} I=\partial I$, are shown in Figure 10. Here the evolution for $\bar{\varkappa}=1$ appears to approach the inner half of a torus, while for $\bar{\varkappa}=-1$ the limiting shape is similar to an hourglass. In both cases the final discrete energy is approximately zero.

In the former case we can prevent the energy converging to zero by requiring the endpoints to remain on lines parallel to the $x_{1}$-axis. I.e. we use the same initial data, but now let $\partial_{S F} I=\partial_{2} I=\partial I$. The evolution is shown in Figure 11, where a numerical steady state is reached with discrete energy $\widehat{E}^{m+1}>25$. For completeness we also display the corresponding simulation with $\bar{\varkappa}=-1$, which is indistinguishable from the one in Figure 10.

Different simulations for the top part of a torus, with clamped boundary conditions at the inner ring, and free boundary conditions at the outer ring, are shown in Figure 12.

Repeating these experiments for Navier boundary conditions at the inner ring of the torus cap, and enforcing surface area preservation, leads to the simulations shown in Figure 13. Here, for $\bar{\varkappa}=1$ we observe pinch-off, and so we stop the simulation just prior to the pinch-off happening. 

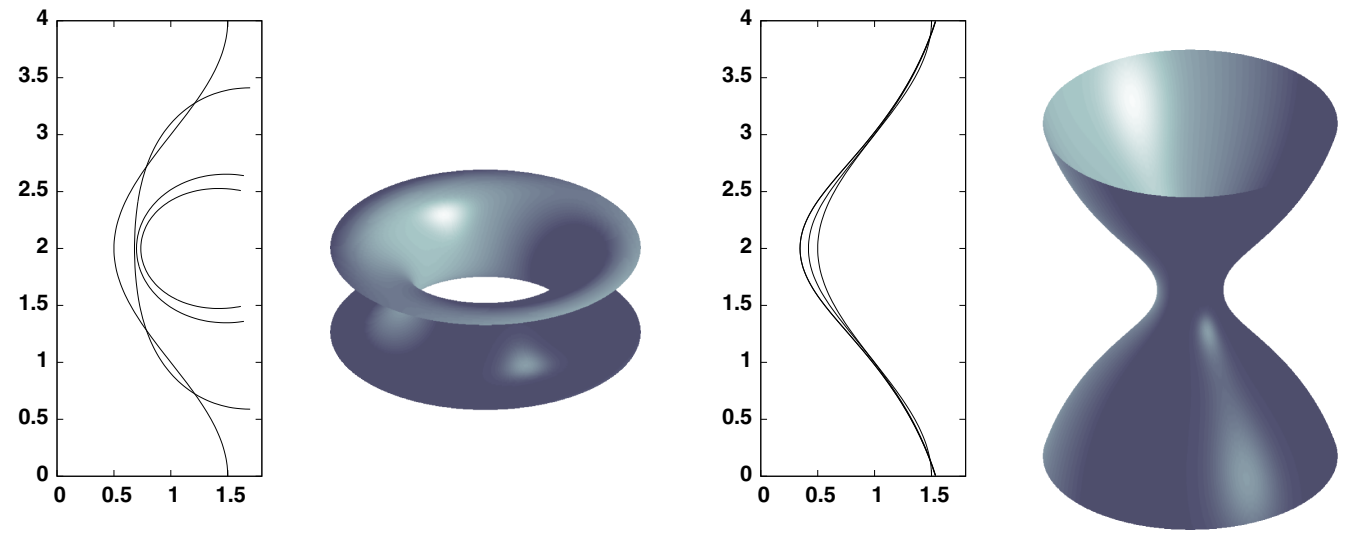

FIgURE 10. $\left(\mathcal{P}^{m}\right)^{h}$ Generalized Willmore flow with free boundary conditions for a dumbbelllike open cylinder shape. Left with $\bar{\varkappa}=1$, right with $\bar{\varkappa}=-1$. The solutions are shown at times $t=0,0.1,0.5,1$, and we also visualize the axisymmetric surfaces generated by $\Gamma^{M}$.
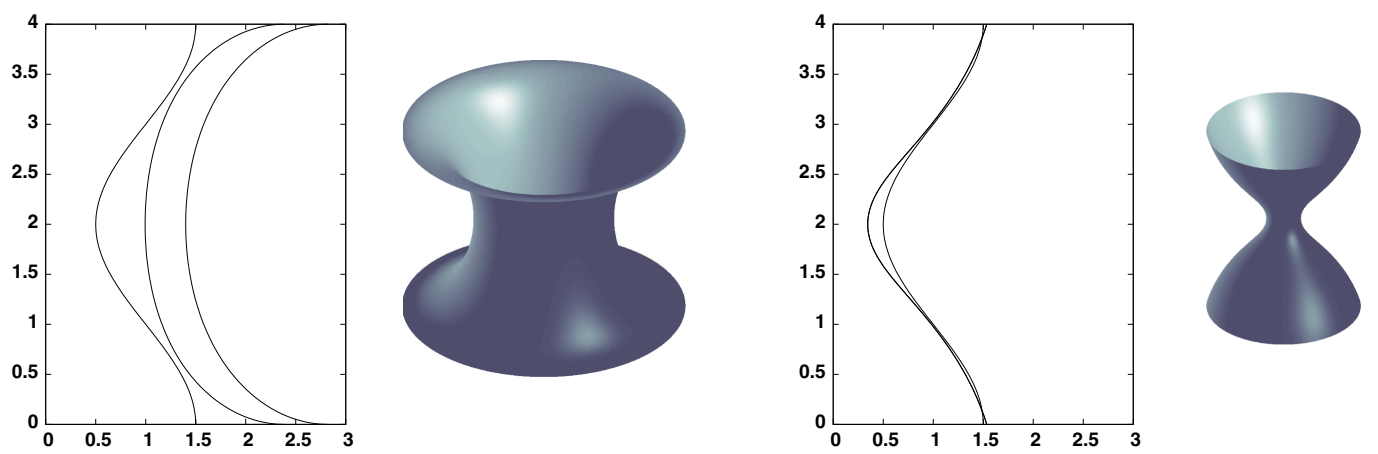

Figure 11. $\left(\mathcal{P}^{m}\right)^{h}$ Generalized Willmore flow with semifree boundary conditions for a dumbbell-like open cylinder shape. Left with $\bar{\varkappa}=1$, right with $\bar{\varkappa}=-1$. The solutions are shown at times $t=0,1,10$, and we also visualize the axisymmetric surfaces generated by $\Gamma^{M}$.
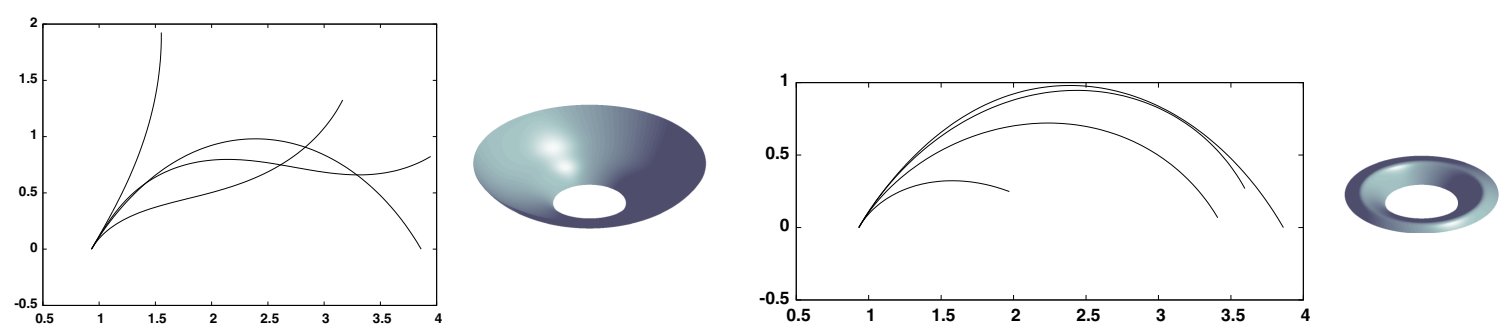

Figure 12. $\left(\mathcal{P}^{m}\right)^{h}$ Generalized Willmore flow with mixed clamped and free boundary conditions for a torus-like cap. Left with $\bar{\varkappa}=1$, right with $\bar{\varkappa}=-1$. The contact angle for the clamped node is chosen as $\vartheta(0)=\frac{7}{6} \pi=210^{\circ}$. The solutions are shown at times $t=0,0.1,1,5$, and we also visualize the axisymmetric surfaces generated by $\Gamma^{M}$. 

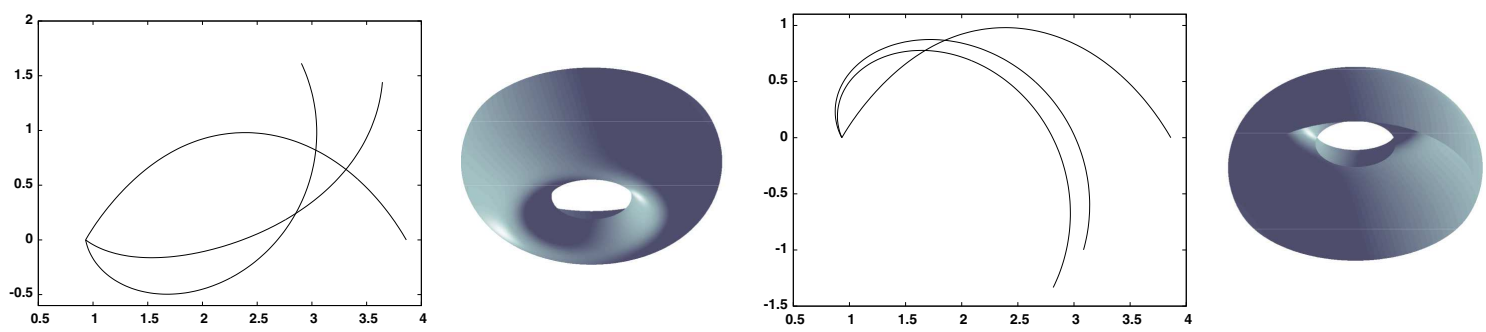

FiguRE 13. $\left(\mathcal{P}^{m}\right)^{h}$ Surface area preserving generalized Willmore flow with mixed Navier and free boundary conditions for a torus-like cap. Left with $\bar{\varkappa}=1$, right with $\bar{\varkappa}=-1$. The solutions are shown at times $t=0,1,5$ (left) and at times $t=0,5,10$ (right), and we also visualize the axisymmetric surfaces generated by $\Gamma^{M}$.
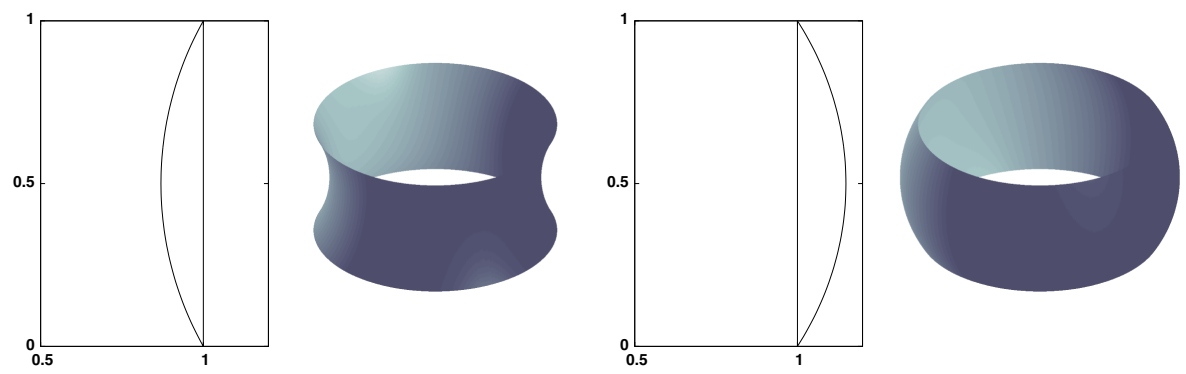

FiguRE 14. $\left(\mathcal{P}^{m}\right)^{h}$ Generalized Willmore flow, for $\alpha_{G}=-1$, with Navier boundary conditions for an open cylinder. Left with $\bar{\varkappa}=1$, right with $\bar{\varkappa}=-1$. The solutions are shown at times $t=0,0.5$, and we also visualize the axisymmetric surfaces generated by $\Gamma^{M}$.

Finally, we show two evolutions for Gaussian curvature effects. To this end, we repeat the simulations in Figure 8 , but now with $\alpha_{G}=-1$. In contrast to the earlier results, the cylindrical initial data is no longer stationary for $\bar{\varkappa}=-1$. See Figure 14 for a visualization of the results.

\section{Appendix A. Consistency of Weak formulations}

\section{A.1. Formulations based on $\varkappa$}

Here we derive the strong form for (3.22), together with possible boundary conditions for the $L^{2}$-gradient flow of (2.21). We recall from (3.12), (3.13), (3.35) and (2.7) that

$$
\vec{y} \cdot \vec{\nu}=2 \pi \vec{x} \cdot \vec{e}_{1}\left[\alpha\left(\varkappa_{\mathcal{S}}-\bar{\varkappa}\right)+\beta \mathcal{A}_{\mathcal{S}}\right], \quad \text { where } \quad \varkappa_{\mathcal{S}}=\varkappa-\frac{\vec{\nu} \cdot \vec{e}_{1}}{\vec{x} \cdot \vec{e}_{1}} \quad \text { and } \mathcal{A}_{\mathcal{S}}=2 \pi\left(\vec{x} \cdot \vec{e}_{1} \varkappa_{\mathcal{S}},\left|\vec{x}_{\rho}\right|\right)-M_{0} \text {. }
$$

We begin by re-stating (3.22a), on noting (A.1) and (3.5b), as

$$
\begin{aligned}
2 \pi\left(\left(\vec{x} \cdot \vec{e}_{1}\right) \vec{x}_{t} \cdot \vec{\nu}, \vec{\chi} \cdot \vec{\nu}\left|\vec{x}_{\rho}\right|\right)= & \left(\vec{y}_{\rho} \cdot \vec{\nu}, \vec{\chi}_{\rho} \cdot \vec{\nu}\left|\vec{x}_{\rho}\right|^{-1}\right)-\pi\left(\alpha\left[\varkappa_{\mathcal{S}}-\bar{\varkappa}\right]^{2}+2 \lambda+2 \beta \mathcal{A}_{\mathcal{S}} \varkappa, \vec{\chi} \cdot \vec{e}_{1}\left|\vec{x}_{\rho}\right|+\left(\vec{x} \cdot \vec{e}_{1}\right) \vec{\chi}_{\rho} \cdot \vec{\tau}\right) \\
& -2 \pi \alpha\left(\varkappa_{\mathcal{S}}-\bar{\varkappa},\left(\varkappa-\varkappa_{\mathcal{S}}\right) \vec{\chi} \cdot \vec{e}_{1}\left|\vec{x}_{\rho}\right|\right)-2 \pi \beta \mathcal{A}_{\mathcal{S}}\left(\vec{e}_{2}, \vec{\chi}_{\rho}\right) \\
& -2 \pi \alpha\left(\varkappa_{\mathcal{S}}-\bar{\varkappa},\left(\vec{\tau} \cdot \vec{e}_{1}\right) \vec{\chi}_{\rho} \cdot \vec{\nu}\right)+\left(\varkappa \vec{y}^{\perp}, \vec{\chi}_{\rho}\right) \\
& -2 \pi \varsigma \sum_{p \in \partial_{S F} I \cup \partial_{F} I} \vec{\chi}(p) \cdot \vec{e}_{1}=\sum_{i=1}^{7} T_{i}(\vec{\chi}) \quad \forall \vec{\chi} \in \mathbb{X} .
\end{aligned}
$$


We now set

$$
\mathcal{B}=\partial_{0} I \cup \partial_{S F} I \cup \partial_{F} I
$$

On recalling $(2.3)$ and $(3.5 \mathrm{~b})$ throughout these $T_{i}(\vec{\chi})$ calculations for any $\vec{\chi} \in \mathbb{X}$, we have that

$$
T_{1}(\vec{\chi})=\left(\left(\vec{y}_{s} \cdot \vec{\nu}\right) \vec{\nu}, \vec{\chi}_{\rho}\right)=-\left(\left[\left(\vec{y}_{s} \cdot \vec{\nu}\right) \vec{\nu}\right]_{s}, \vec{\chi}\left|\vec{x}_{\rho}\right|\right)+\sum_{p \in \mathcal{B}}(-1)^{p+1}\left[\left(\vec{y}_{s} \cdot \vec{\nu}\right) \vec{\chi} \cdot \vec{\nu}\right](p)=A_{1}(\vec{\chi})+B_{1}(\vec{\chi}) .
$$

Moreover,

$$
\begin{aligned}
\sum_{i=2}^{4} T_{i}(\vec{\chi})=- & \pi\left(\alpha\left(\varkappa_{\mathcal{S}}-\bar{\varkappa}\right)\left(2 \varkappa-\varkappa_{\mathcal{S}}-\bar{\varkappa}\right)+2 \lambda+2 \beta \mathcal{A}_{\mathcal{S}} \varkappa, \vec{\chi} \cdot \vec{e}_{1}\left|\vec{x}_{\rho}\right|\right) \\
& -\pi\left(\vec{x} \cdot \vec{e}_{1}\left(\alpha\left[\varkappa_{\mathcal{S}}-\bar{\varkappa}\right]^{2}+2 \lambda+2 \beta \mathcal{A}_{\mathcal{S}} \varkappa\right) \vec{\tau}+2 \beta \mathcal{A}_{\mathcal{S}} \vec{e}_{2}, \vec{\chi}_{\rho}\right)=A_{2}(\vec{\chi})+T_{8}(\vec{\chi}),
\end{aligned}
$$

where

$$
\begin{aligned}
T_{8}(\vec{\chi})= & \pi\left(\left[\vec{x} \cdot \vec{e}_{1}\left(\alpha\left[\varkappa_{\mathcal{S}}-\bar{\varkappa}\right]^{2}+2 \lambda+2 \beta \mathcal{A}_{\mathcal{S}} \varkappa\right) \vec{\tau}\right]_{s}, \vec{\chi}\left|\vec{x}_{\rho}\right|\right) \\
& -\pi \sum_{p \in \mathcal{B}}(-1)^{p+1}\left[\vec{x} \cdot \vec{e}_{1}\left(\alpha\left[\varkappa_{\mathcal{S}}-\bar{\varkappa}\right]^{2}+2 \lambda+2 \beta \mathcal{A}_{\mathcal{S}} \varkappa\right) \vec{\chi} \cdot \vec{\tau}+2 \beta \mathcal{A}_{\mathcal{S}} \vec{\chi} \cdot \vec{e}_{2}\right](p)=A_{3}(\vec{\chi})+B_{3}(\vec{\chi}) .
\end{aligned}
$$

In addition,

$$
T_{5}(\vec{\chi})=2 \pi \alpha\left(\left[\left(\varkappa_{\mathcal{S}}-\bar{\varkappa}\right)\left(\vec{\tau} \cdot \vec{e}_{1}\right) \vec{\nu}\right]_{S}, \vec{\chi}\left|\vec{x}_{\rho}\right|\right)-2 \pi \alpha \sum_{p \in \mathcal{B}}(-1)^{p+1}\left[\left(\varkappa_{\mathcal{S}}-\bar{\varkappa}\right)\left(\vec{\tau} \cdot \vec{e}_{1}\right) \vec{\chi} \cdot \vec{\nu}\right](p)=A_{4}(\vec{\chi})+B_{4}(\vec{\chi}) .
$$

Finally,

$$
T_{6}(\vec{\chi})=-\left(\left(\varkappa \vec{y}^{\perp}\right)_{s}, \vec{\chi}\left|\vec{x}_{\rho}\right|\right)+\sum_{p \in \mathcal{B}}(-1)^{p+1}\left[\varkappa \vec{\chi} \cdot \vec{y}^{\perp}\right](p)=A_{5}(\vec{\chi})+B_{5}(\vec{\chi}),
$$

and $B_{2}(\vec{\chi})=T_{7}(\vec{\chi})$. With the above definitions, we can write $($ A.2) as

$$
2 \pi\left(\left(\vec{x} \cdot \vec{e}_{1}\right) \vec{x}_{t} \cdot \vec{\nu}, \vec{\chi} \cdot \vec{\nu}\left|\vec{x}_{\rho}\right|\right)=\sum_{i=1}^{7} T_{i}(\vec{\chi})=\sum_{i=1}^{5} A_{i}(\vec{\chi})+\sum_{i=1}^{5} B_{i}(\vec{\chi}) \quad \forall \vec{\chi} \in \mathbb{X} .
$$

It follows from (3.23), (3.24) and

$$
\vec{\nu}_{s}=-\varkappa \vec{\tau}
$$

that

$$
\begin{aligned}
\left(\left(\vec{y}_{s} \cdot \vec{\nu}\right) \vec{\nu}+\varkappa \vec{y}^{\perp}\right)_{s} & =\left((\vec{y} \cdot \vec{\nu})_{s} \vec{\nu}+\varkappa(\vec{y} \cdot \vec{\nu}) \vec{\tau}\right)_{s}=(\vec{y} \cdot \vec{\nu})_{s s} \vec{\nu}+(\vec{y} \cdot \vec{\nu})_{s} \vec{\nu}_{s}+\varkappa(\vec{y} \cdot \vec{\nu})_{s} \vec{\tau}+(\vec{y} \cdot \vec{\nu})(\varkappa \vec{\tau})_{s} \\
& =(\vec{y} \cdot \vec{\nu})_{s s} \vec{\nu}+(\vec{y} \cdot \vec{\nu})(\varkappa \vec{\tau})_{s},
\end{aligned}
$$

and so

$$
A_{1}(\vec{\chi})+A_{5}(\vec{\chi})=-\left(\left[(\vec{y} \cdot \vec{\nu})_{s s}+\vec{y} \cdot \vec{\nu} \varkappa^{2}\right] \vec{\nu}+\vec{y} \cdot \vec{\nu} \varkappa_{s} \vec{\tau}, \vec{\chi}\left|\vec{x}_{\rho}\right|\right) .
$$

Choosing $\vec{\chi}=\chi \vec{\tau}$, for $\chi \in H_{0}^{1}(I)$, in (A.9), and combining (A.4)-(A.8) and (A.11), we obtain for the right hand side of (A.9)

$$
\sum_{i=1}^{7} T_{i}(\chi \vec{\tau})=\sum_{i=1}^{5} A_{i}(\chi \vec{\tau})=-\left(\vec{y} \cdot \vec{\nu} \varkappa_{s}+\pi\left[\alpha\left(\varkappa_{\mathcal{S}}-\bar{\varkappa}\right)\left(2 \varkappa-\varkappa_{\mathcal{S}}-\bar{\varkappa}\right)+2 \lambda+2 \beta \mathcal{A}_{\mathcal{S}} \varkappa\right] \vec{\tau} \cdot \vec{e}_{1}, \chi\left|\vec{x}_{\rho}\right|\right)
$$




$$
\begin{aligned}
& +\pi\left(\left[\vec{x} \cdot \vec{e}_{1}\left(\alpha\left[\varkappa_{\mathcal{S}}-\bar{\varkappa}\right]^{2}+2 \lambda+2 \beta \mathcal{A}_{\mathcal{S}} \varkappa\right)\right]_{S}-2 \alpha\left(\varkappa_{\mathcal{S}}-\bar{\varkappa}\right) \vec{\tau} \cdot \vec{e}_{1} \varkappa, \chi\left|\vec{x}_{\rho}\right|\right) \\
= & -\pi \alpha\left(\vec{x} \cdot \vec{e}_{1}\left(2\left(\varkappa_{\mathcal{S}}-\bar{\varkappa}\right) \varkappa_{\mathcal{S}}-\left[\left[\varkappa_{\mathcal{S}}-\bar{\varkappa}\right]^{2}\right]_{S}\right), \chi\left|\vec{x}_{\rho}\right|\right)+2 \pi \alpha\left(\left(\varkappa_{\mathcal{S}}-\bar{\varkappa}\right)\left(\varkappa_{\mathcal{S}}-2 \varkappa\right), \vec{\tau} \cdot \vec{e}_{1} \chi\left|\vec{x}_{\rho}\right|\right) \\
= & 2 \pi \alpha\left(\left(\varkappa_{\mathcal{S}}-\bar{\varkappa}\right)\left[\vec{x} \cdot \vec{e}_{1}\left(\varkappa_{\mathcal{S}}-\varkappa\right)_{s}+\vec{\tau} \cdot \vec{e}_{1}\left(\varkappa_{\mathcal{S}}-2 \varkappa\right)\right], \chi\left|\vec{x}_{\rho}\right|\right),
\end{aligned}
$$

where we have noted that $\vec{\tau}_{s} \cdot \vec{\tau}=0,($ A.10), (2.3) and (A.1). In addition, it holds, on noting (A.1), (2.3) and (A.10), that

$$
\vec{x} \cdot \vec{e}_{1}\left(\varkappa_{\mathcal{S}}-\varkappa\right)_{s}=-\vec{x} \cdot \vec{e}_{1}\left[\frac{\vec{\nu} \cdot \vec{e}_{1}}{\vec{x} \cdot \vec{e}_{1}}\right]_{s}=\frac{\left(\vec{\nu} \cdot \vec{e}_{1}\right) \vec{\tau} \cdot \vec{e}_{1}-\left(\vec{\nu}_{s} \cdot \vec{e}_{1}\right) \vec{x} \cdot \vec{e}_{1}}{\vec{x} \cdot \vec{e}_{1}}=\vec{\tau} \cdot \vec{e}_{1}\left(2 \varkappa-\varkappa_{\mathcal{S}}\right),
$$

and so substituting into (A.12) yields that $\sum_{i=1}^{7} T_{i}(\chi \vec{\tau})=0$, as expected.

Choosing $\vec{\chi}=\chi \vec{\nu}$, for $\chi \in H_{0}^{1}(I)$, in (A.9), and combining (A.4)-(A.8) and (A.11), we obtain for the right hand side of (A.9)

$$
\begin{aligned}
& \sum_{i=1}^{7} T_{i}(\chi \vec{\nu})=\sum_{i=1}^{5} A_{i}(\chi \vec{\nu})=-\left((\vec{y} \cdot \vec{\nu})_{s s}+\vec{y} \cdot \vec{\nu} \varkappa^{2}, \chi\left|\vec{x}_{\rho}\right|\right) \\
& -\pi\left(\left[\alpha\left(\varkappa_{\mathcal{S}}-\bar{\varkappa}\right)\left(2 \varkappa-\varkappa_{\mathcal{S}}-\bar{\varkappa}\right)+2 \lambda+2 \beta \mathcal{A}_{\mathcal{S}} \varkappa\right] \vec{\nu} \cdot \vec{e}_{1}, \chi\left|\vec{x}_{\rho}\right|\right) \\
& +\pi\left(\vec{x} \cdot \vec{e}_{1}\left(\alpha\left[\varkappa_{\mathcal{S}}-\bar{\varkappa}\right]^{2}+2 \lambda+2 \beta \mathcal{A}_{\mathcal{S}} \varkappa\right) \varkappa, \chi\left|\vec{x}_{\rho}\right|\right)+2 \pi \alpha\left(\left[\left(\varkappa_{\mathcal{S}}-\bar{\varkappa}\right) \vec{\tau} \cdot \vec{e}_{1}\right]_{s}, \chi\left|\vec{x}_{\rho}\right|\right) \\
& =-2 \pi\left(\left(\vec{x} \cdot \vec{e}_{1}\left[\alpha\left(\varkappa_{\mathcal{S}}-\bar{\varkappa}\right)+\beta \mathcal{A}_{\mathcal{S}}\right]\right)_{s S}+\vec{x} \cdot \vec{e}_{1}\left[\alpha\left(\varkappa_{\mathcal{S}}-\bar{\varkappa}\right)+\beta \mathcal{A}_{\mathcal{S}}\right] \varkappa^{2}, \chi\left|\vec{x}_{\rho}\right|\right) \\
& +\pi\left(\left[\alpha\left[\varkappa_{\mathcal{S}}^{2}-\bar{\varkappa}^{2}\right]-2 \lambda-2 \beta \mathcal{A}_{\mathcal{S}} \varkappa\right] \vec{\nu} \cdot \vec{e}_{1}, \chi\left|\vec{x}_{\rho}\right|\right) \\
& +\pi\left(\vec{x} \cdot \vec{e}_{1}\left[\alpha\left[\varkappa_{\mathcal{S}}-\bar{\varkappa}\right]^{2}+2 \lambda+2 \beta \mathcal{A}_{\mathcal{S}} \varkappa\right] \varkappa, \chi\left|\vec{x}_{\rho}\right|\right)+2 \pi \alpha\left(\left(\varkappa_{\mathcal{S}}\right)_{s} \vec{\tau} \cdot \vec{e}_{1}, \chi\left|\vec{x}_{\rho}\right|\right) \\
& =-2 \pi\left(\alpha \vec{x} \cdot \vec{e}_{1}\left(\varkappa_{\mathcal{S}}\right)_{s s}+\alpha \vec{\tau} \cdot \vec{e}_{1}\left(\varkappa_{\mathcal{S}}\right)_{s}+\varkappa \vec{\nu} \cdot \vec{e}_{1}\left[\alpha\left(\varkappa_{\mathcal{S}}-\bar{\varkappa}\right)+\beta \mathcal{A}_{\mathcal{S}}\right], \chi\left|\vec{x}_{\rho}\right|\right) \\
& -2 \pi\left(\vec{x} \cdot \vec{e}_{1}\left[\alpha\left(\varkappa_{\mathcal{S}}-\bar{\varkappa}\right)+\beta \mathcal{A}_{\mathcal{S}}\right] \varkappa^{2}, \chi\left|\vec{x}_{\rho}\right|\right)+\pi\left(\vec{x} \cdot \vec{e}_{1}\left[\alpha\left[\varkappa_{\mathcal{S}}^{2}-\bar{\varkappa}^{2}\right]-2 \lambda-2 \beta \mathcal{A}_{\mathcal{S}} \varkappa\right]\left(\varkappa-\varkappa_{\mathcal{S}}\right), \chi\left|\vec{x}_{\rho}\right|\right) \\
& +\pi\left(\vec{x} \cdot \vec{e}_{1}\left[\alpha\left[\varkappa_{\mathcal{S}}-\bar{\varkappa}\right]^{2}+2 \lambda+2 \beta \mathcal{A}_{\mathcal{S}} \varkappa\right] \varkappa, \chi\left|\vec{x}_{\rho}\right|\right) \\
& =-2 \pi \alpha\left(\left(\vec{x} \cdot \vec{e}_{1}\left(\varkappa_{\mathcal{S}}\right)_{s}\right)_{s}, \chi\left|\vec{x}_{\rho}\right|\right)+2 \pi \lambda\left(\vec{x} \cdot \vec{e}_{1} \varkappa_{\mathcal{S}}, \chi\left|\vec{x}_{\rho}\right|\right)+\pi \alpha\left(\vec{x} \cdot \vec{e}_{1}\left[\varkappa_{\mathcal{S}}^{2}-\bar{\varkappa}^{2}\right]\left(\varkappa-\varkappa_{\mathcal{S}}\right), \chi\left|\vec{x}_{\rho}\right|\right) \\
& \left.+4 \pi \beta \mathcal{A}_{\mathcal{S}}\left(\vec{x} \cdot \vec{e}_{1}, \varkappa_{\mathcal{S}}-\varkappa\right), \chi\left|\vec{x}_{\rho}\right|\right)+\pi \alpha\left(\vec{x} \cdot \vec{e}_{1}\left(\varkappa_{\mathcal{S}}-\bar{\varkappa}\right)\left[2\left(\varkappa_{\mathcal{S}}-\varkappa\right) \varkappa-2 \varkappa^{2}+\left(\varkappa_{\mathcal{S}}-\bar{\varkappa}\right) \varkappa\right], \chi\left|\vec{x}_{\rho}\right|\right) \\
& =-2 \pi \alpha\left(\left(\vec{x} \cdot \vec{e}_{1}\left(\varkappa_{\mathcal{S}}\right)_{s}\right)_{s}, \chi\left|\vec{x}_{\rho}\right|\right)+2 \pi \lambda\left(\vec{x} \cdot \vec{e}_{1} \varkappa_{\mathcal{S}}, \chi\left|\vec{x}_{\rho}\right|\right)-\pi \alpha\left(\vec{x} \cdot \vec{e}_{1}\left[\varkappa_{\mathcal{S}}^{2}-\bar{\varkappa}^{2}\right] \varkappa_{\mathcal{S}}, \chi\left|\vec{x}_{\rho}\right|\right) \\
& +4 \pi \beta \mathcal{A}_{\mathcal{S}}\left(\vec{x} \cdot \vec{e}_{1}, \varkappa\left(\varkappa_{\mathcal{S}}-\varkappa\right), \chi\left|\vec{x}_{\rho}\right|\right)+4 \pi \alpha\left(\vec{x} \cdot \vec{e}_{1}\left(\varkappa_{\mathcal{S}}-\bar{\varkappa}\right)\left(\varkappa_{\mathcal{S}}-\varkappa\right) \varkappa, \chi\left|\vec{x}_{\rho}\right|\right),
\end{aligned}
$$

where we have noted that $\vec{\nu}_{s} \cdot \vec{\nu}=0,(2.6),($ A.1) and (2.3). Clearly, it follows from (A.2), (A.13) and (2.7) that (2.24) holds.

It remains to show that the weak formulation (3.22) indeed enforces the desired boundary conditions. Here, we recall that apart from the axisymmetric boundary conditions (2.35) on $\partial_{0} I$, we consider the following four different types of boundary conditions on $\partial I \backslash \partial_{0} I$.

(i) $\partial \mathcal{S}(t)$ is free, i.e. $\partial_{F} \mathcal{S}(t)$, see $(2.25)$ for $\partial \mathcal{S}(t)$ and $(2.30)$ in the axisymmetric case.

(ii) $\partial \mathcal{S}(t) \subset \partial \mathcal{D}$ is semifree, i.e. $\partial_{S F} \mathcal{S}(t)$, see $(2.27)$ for $\partial \mathcal{S}(t)$ and $(2.31)$ in the axisymmetric case.

(iii) $\partial \mathcal{S}(t)$ clamped, i.e. $\partial_{C} \mathcal{S}(t)$, see $(2.28)$ for $\partial \mathcal{S}(t)$ and $(2.33)$ in the axisymmetric case.

(iv) $\partial \mathcal{S}(t)$ having Navier conditions, i.e. $\partial_{N} \mathcal{S}(t)$, see $(2.29)$ for $\partial \mathcal{S}(t)$ and (2.34) in the axisymmetric case.

Firstly, we recall from (3.22) that $\vec{x}(\cdot, t) \in \underline{V}_{\partial_{0}}$ with $\vec{x}_{t}(\cdot, t) \in \mathbb{X}$, for $t \in(0, T]$, imposes strongly that (2.31a), (2.33a), (2.34a) and (2.35a) hold. Furthermore, we note that (3.22c) weakly imposes (2.35b) and (2.33b), recall the paragraph below (3.8). This means that we still need to show (2.35c), (2.30), (2.31b), (2.31c) and (2.34b). 
We begin with (2.35c). To this end, we choose test functions $\vec{\chi}=\chi \vec{\nu}$ in (A.2), where $\chi \in H^{1}(I)$ is zero away from $\partial_{0} I$, to obtain

$$
\begin{aligned}
2 \pi & \left(\left(\vec{x} \cdot \vec{e}_{1}\right) \vec{x}_{t} \cdot \vec{\nu}, \chi\left|\vec{x}_{\rho}\right|\right)=\left(\vec{y}_{\rho} \cdot \vec{\nu}, \chi_{\rho}\left|\vec{x}_{\rho}\right|^{-1}\right)-\pi\left(\alpha\left[\varkappa_{\mathcal{S}}-\bar{\varkappa}\right]^{2}+2 \lambda+2 \beta \mathcal{A}_{\mathcal{S}} \varkappa, \chi \vec{\nu} \cdot \vec{e}_{1}\left|\vec{x}_{\rho}\right|+\left(\vec{x} \cdot \vec{e}_{1}\right) \vec{\tau} \cdot \vec{\nu}_{\rho} \chi\right) \\
& -2 \pi \alpha\left(\varkappa_{\mathcal{S}}-\bar{\varkappa},\left(\varkappa-\varkappa_{\mathcal{S}}\right) \chi \vec{\nu} \cdot \vec{e}_{1}\left|\vec{x}_{\rho}\right|\right)-2 \pi \alpha\left(\varkappa_{\mathcal{S}}-\bar{\varkappa},\left(\vec{\tau} \cdot \vec{e}_{1}\right) \chi_{\rho}\right)+\left(\varkappa \vec{y}^{\perp}-2 \pi \beta \mathcal{A}_{\mathcal{S}} \vec{e}_{2}, \chi_{\rho} \vec{\nu}+\chi \vec{\nu}_{\rho}\right) .
\end{aligned}
$$

We are interested in the boundary condition that is weakly enforced by (A.14) on $\partial_{0} I$, and so the only relevant terms are the ones involving $\chi_{\rho}$ on the right hand side of (A.14). They simplify, on noting from (2.3) that $\vec{\nu} \cdot \vec{e}_{2}=\vec{\tau} \cdot \vec{e}_{1}$, and on recalling (3.24) and (A.1), to

$$
\begin{aligned}
\left(\vec{y}_{\rho} \cdot\right. & \left.\vec{\nu}, \chi_{\rho}\left|\vec{x}_{\rho}\right|^{-1}\right)-2 \pi \alpha\left(\varkappa_{\mathcal{S}}-\bar{\varkappa},\left(\vec{\tau} \cdot \vec{e}_{1}\right) \chi_{\rho}\right)+\left(\varkappa \vec{y}^{\perp}-2 \pi \beta \mathcal{A}_{\mathcal{S}} \vec{e}_{2}, \chi_{\rho} \vec{\nu}\right) \\
& =\left(\vec{y}_{s} \cdot \vec{\nu}-2 \pi\left(\alpha\left(\varkappa_{\mathcal{S}}-\bar{\varkappa}\right)+\beta \mathcal{A}_{\mathcal{S}}\right)\left(\vec{\tau} \cdot \vec{e}_{1}\right)+\varkappa \vec{y}^{\perp} \cdot \vec{\nu}, \chi_{\rho}\right) \\
& =\left((\vec{y} \cdot \vec{\nu})_{s}-2 \pi\left(\alpha\left(\varkappa_{\mathcal{S}}-\bar{\varkappa}\right)+\beta \mathcal{A}_{\mathcal{S}}\right)\left(\vec{\tau} \cdot \vec{e}_{1}\right), \chi_{\rho}\right)=2 \pi \alpha\left(\vec{x} \cdot \vec{e}_{1}\left(\varkappa_{\mathcal{S}}\right)_{\rho}, \chi_{\rho}\left|\vec{x}_{\rho}\right|^{-1}\right) .
\end{aligned}
$$

We can now argue as in Appendix A.1 of [7] to show that despite the degenerate weight in the last term in (A.15), the identity (A.14) gives rise to the boundary condition $\left(\varkappa_{\mathcal{S}}\right)_{\rho}=0$ on $\partial_{0} I$. In particular, we note that all the integrands in all the remaining terms converge to zero for $\rho$ approaching $\partial_{0} I$, on recalling (2.10). This proves that $(3.22 \mathrm{a})$ weakly enforces $(2.35 \mathrm{c})$.

Next we recall from (2.37) and (3.15) that

$$
\vec{y}=2 \pi \alpha_{G} \vec{e}_{1} \quad \text { on } \quad \partial_{M} I=\partial_{N} I \cup \partial_{S F} I \cup \partial_{F} I .
$$

Combining with (A.1) yields that

$$
\alpha\left(\varkappa_{\mathcal{S}}-\bar{\varkappa}\right)+\beta \mathcal{A}_{\mathcal{S}}-\alpha_{G} \frac{\vec{\nu} \cdot \vec{e}_{1}}{\vec{x} \cdot \vec{e}_{1}}=0 \quad \text { on } \quad \partial_{M} I .
$$

Hence, we have that (2.30c), (2.31c) and (2.34b) are imposed strongly. Overall, we still need to show (2.30a), $(2.30 \mathrm{~b})$ and $(2.31 \mathrm{~b})$. To this end, we derive conditions that make the second sum in (A.9) vanish for all test functions $\vec{\chi} \in \mathbb{X}$. It follows from (A.4) and (A.8), on recalling (3.24) and (A.1), that

$$
\begin{aligned}
B_{1}(\vec{\chi})+B_{5}(\vec{\chi})= & \sum_{p \in \mathcal{B}}(-1)^{p+1}\left[\left(\left(\vec{y}_{s} \cdot \vec{\nu}\right) \vec{\nu}+\varkappa \vec{y}^{\perp}\right) \cdot \vec{\chi}\right](p)=\sum_{p \in \mathcal{B}}(-1)^{p+1}\left[\left((\vec{y} \cdot \vec{\nu})_{s} \vec{\nu}+\varkappa(\vec{y} \cdot \vec{\nu}) \vec{\tau}\right) \cdot \vec{\chi}\right](p) \\
= & 2 \pi \sum_{p \in \mathcal{B}}(-1)^{p+1}\left[\left(\vec{x} \cdot \vec{e}_{1}\left[\alpha\left(\varkappa_{\mathcal{S}}-\bar{\varkappa}\right)+\beta \mathcal{A}_{\mathcal{S}}\right]\right)_{s} \vec{\chi} \cdot \vec{\nu}\right](p) \\
& +2 \pi \sum_{p \in \partial_{S F} I \cup \partial_{F} I}(-1)^{p+1}\left[\varkappa\left(\vec{x} \cdot \vec{e}_{1}\left[\alpha\left(\varkappa_{\mathcal{S}}-\bar{\varkappa}\right)+\beta \mathcal{A}_{\mathcal{S}}\right]\right) \vec{\chi} \cdot \vec{\tau}\right](p),
\end{aligned}
$$

where in the last term we have noted that (2.35b) implies that $\vec{\nu} \cdot \vec{e}_{1}=0$ on $\partial_{0} I$, recall also (2.10). Looking first at the normal boundary contributions, we compute for a $\chi \in H^{1}(I)$, on noting from (2.3) that $\vec{\nu} \cdot \vec{e}_{2}=\vec{\tau} \cdot \vec{e}_{1}$, that

$$
\begin{aligned}
\sum_{i=1}^{5} B_{i}(\chi \vec{\nu})= & 2 \pi \sum_{p \in \mathcal{B}}(-1)^{p+1}\left[\left(\vec{x} \cdot \vec{e}_{1}\left[\alpha\left(\varkappa_{\mathcal{S}}-\bar{\varkappa}\right)+\beta \mathcal{A}_{\mathcal{S}}\right]\right)_{s} \chi\right](p) \\
& -2 \pi \varsigma \sum_{p \in \partial_{S F} I \cup \partial_{F} I}\left[\vec{\nu} \cdot \vec{e}_{1} \chi\right](p)-2 \pi \beta \mathcal{A}_{\mathcal{S}} \sum_{p \in \mathcal{B}}(-1)^{p+1}\left[\vec{\nu} \cdot \vec{e}_{2} \chi\right](p) \\
& -2 \pi \alpha \sum_{p \in \mathcal{B}}(-1)^{p+1}\left[\left(\varkappa_{\mathcal{S}}-\bar{\varkappa}\right) \vec{\tau} \cdot \vec{e}_{1} \chi\right](p)
\end{aligned}
$$




$$
\begin{aligned}
& =2 \pi \alpha \sum_{p \in \mathcal{B}}(-1)^{p+1}\left[\vec{x} \cdot \vec{e}_{1}\left(\varkappa_{\mathcal{S}}\right)_{s} \chi\right](p)-2 \pi \varsigma \sum_{p \in \partial_{S F} I \cup \partial_{F} I}\left[\vec{x} \cdot \vec{e}_{1} \frac{\vec{\nu} \cdot \vec{e}_{1}}{\vec{x} \cdot \vec{e}_{1}} \chi\right](p) \\
& =2 \pi \sum_{p \in \partial_{S F} I \cup \partial_{F} I}\left[\vec{x} \cdot \vec{e}_{1}\left((-1)^{p+1} \alpha\left(\varkappa_{\mathcal{S}}\right)_{s}-\varsigma \frac{\vec{\nu} \cdot \vec{e}_{1}}{\vec{x} \cdot \vec{e}_{1}}\right) \chi\right](p) \quad \forall \chi \in H^{1}(I),
\end{aligned}
$$

where in the last step we have observed (2.35c) and (A.3). This gives (2.30a) on $\partial_{F} I$, on recalling (2.2a) and (3.5b).

Next we consider the tangential components. It holds, on noting (A.16), (2.7), $\vec{\nu} \cdot \vec{e}_{1}=-\vec{\tau} \cdot \vec{e}_{2},(2.12),(2.20)$ and $(2.2 \mathrm{~b})$, that

$$
\begin{aligned}
\sum_{i=1}^{5} & B_{i}(\chi \vec{\tau})=2 \pi \sum_{p \in \partial_{S F} I \cup \partial_{F} I}(-1)^{p+1}\left[\varkappa\left(\vec{x} \cdot \vec{e}_{1}\left[\alpha\left(\varkappa_{\mathcal{S}}-\bar{\varkappa}\right)+\beta \mathcal{A}_{\mathcal{S}}\right]\right) \chi\right](p) \\
& -2 \pi \varsigma \sum_{p \in \partial_{S F} \cup \partial_{F} I}\left[\vec{\tau} \cdot \vec{e}_{1} \chi\right](p)-2 \pi \beta \mathcal{A}_{\mathcal{S}} \sum_{p \in \mathcal{B}}(-1)^{p+1}\left[\vec{\tau} \cdot \vec{e}_{2} \chi\right](p) \\
& -\pi \sum_{p \in \partial_{S F} I \cup \partial_{F} I}(-1)^{p+1}\left[\vec{x} \cdot \vec{e}_{1}\left(\alpha\left[\varkappa \mathcal{S}-\bar{\varkappa}^{2}+2 \lambda+2 \beta \mathcal{A}_{\mathcal{S}} \varkappa\right) \chi\right](p)\right. \\
= & -2 \pi \alpha_{G} \sum_{p \in \partial_{S F} I \cup \partial_{F} I}(-1)^{p+1}\left[\vec{x} \cdot \vec{e}_{1} \mathcal{K}_{\mathcal{S}} \chi\right](p)-2 \pi \varsigma \sum_{p \in \partial_{S F} \cup \partial_{F} I}(-1)^{p+1}\left[\vec{x} \cdot \vec{e}_{1} \frac{\vec{\mu} \cdot \vec{e}_{1}}{\vec{x} \cdot \vec{e}_{1}} \chi\right](p) \\
& +2 \pi \beta \mathcal{A}_{\mathcal{S}} \sum_{p \in \partial_{S F} I \cup \partial_{F} I}(-1)^{p+1}\left[\vec{x} \cdot \vec{e}_{1} \frac{\vec{\nu} \cdot \vec{e}_{1}}{\vec{x} \cdot \vec{e}_{1}} \chi\right](p) \sum_{p \in \partial_{S F} I \cup \partial_{F} I}(-1)^{p+1}\left[\vec{x} \cdot \vec{e}_{1}\left(\frac{1}{2} \alpha\left[\varkappa_{\mathcal{S}}-\bar{\varkappa}\right]^{2}+\lambda+\beta \mathcal{A}_{\mathcal{S}} \varkappa\right) \chi\right](p) \\
& -2 \pi \sum_{p \in \partial_{S F} \cup \partial_{F} I}(-1)^{p+1}\left[\vec{x} \cdot \vec{e}_{1} \frac{\vec{\mu} \cdot \vec{e}_{1}}{\vec{x} \cdot \vec{e}_{1}} \chi\right](p) \\
& -2 \pi \varsigma \sum_{p \in \partial_{S F} I \cup \partial_{F} I}(-1)^{p+1}\left[\vec{x} \cdot \vec{e}_{1}\left(\alpha_{G} \mathcal{K}_{\mathcal{S}}+\frac{1}{2} \alpha\left[\varkappa \mathcal{S}-\bar{\varkappa}^{2}+\lambda+\beta \mathcal{A}_{\mathcal{S}} \varkappa_{\mathcal{S}}\right) \chi\right](p)\right. \\
& -2 \pi \sum_{p \in \partial_{S F} \cup \partial_{F} I}(-1)^{p}\left[\vec{x} \cdot \vec{e}_{1}\left(\varsigma \frac{\vec{\mu} \cdot \vec{e}_{1}}{\vec{x} \cdot \vec{e}_{1}}+\alpha_{G} \mathcal{K}_{\mathcal{S}}+\frac{1}{2} \alpha[\varkappa \mathcal{S}-\bar{\varkappa}]^{2}+\lambda+\beta \mathcal{A}_{\mathcal{S}} \varkappa \mathcal{S}\right) \chi\right](p) \quad \forall \chi \in H^{1}(I) .
\end{aligned}
$$

This yields $(2.30 \mathrm{~b})$ on $\partial_{F} I$, on recalling $(2.2 \mathrm{a})$ and (3.5b). In order to prove $(2.31 \mathrm{~b})$ on $\partial_{S F} I$, we choose a test function $\vec{\chi} \in \mathbb{X}$ and then combine (A.17) and (A.18). For example, on $\partial_{1} I$ we choose $\vec{\chi}=\chi \vec{e}_{2}=$ $\chi\left(\vec{\nu} \cdot \vec{e}_{2}\right) \vec{\nu}+\chi\left(\vec{\tau} \cdot \vec{e}_{2}\right) \vec{\tau}=\chi(-1)^{p+1}\left(\vec{\mu} \cdot \vec{e}_{1}\right) \vec{\nu}+\chi\left(-\vec{\nu} \cdot \vec{e}_{1}\right) \vec{\tau}$, and hence we obtain the desired result. The case $\partial_{2} I$ follows analogously.

\section{A.2. Formulations based on $\varkappa_{\mathcal{S}}$}

Here we derive the strong form for (3.44), together with possible boundary conditions for the $L^{2}$-gradient flow of (2.21). We recall from (3.34) and (3.35) that

$$
\vec{y}_{\mathcal{S}} \cdot \vec{\nu}=2 \pi\left[\alpha\left(\varkappa_{\mathcal{S}}-\bar{\varkappa}\right)+\beta \mathcal{A}_{\mathcal{S}}\right], \quad \text { where } \mathcal{A}_{\mathcal{S}}=2 \pi\left(\vec{x} \cdot \vec{e}_{1} \varkappa_{\mathcal{S}},\left|\vec{x}_{\rho}\right|\right)-M_{0} .
$$

We begin by re-stating (3.44a), on noting (2.3), as 


$$
\begin{aligned}
2 \pi\left(\left(\vec{x} \cdot \vec{e}_{1}\right) \vec{x}_{t} \cdot \vec{\nu}, \vec{\chi} \cdot \vec{\nu}\left|\vec{x}_{\rho}\right|\right)= & \left(\left(\vec{x} \cdot \vec{e}_{1}\right)\left(\vec{y}_{\mathcal{S}}\right)_{\rho} \cdot \vec{\nu}, \vec{\chi}_{\rho} \cdot \vec{\nu}\left|\vec{x}_{\rho}\right|^{-1}\right) \\
& -\left(\pi \vec{x} \cdot \vec{e}_{1}\left[\alpha\left(\varkappa_{\mathcal{S}}-\bar{\varkappa}\right)^{2}+2 \lambda+2 \beta \mathcal{A}_{\mathcal{S}} \varkappa_{\mathcal{S}}\right]-\vec{y}_{\mathcal{S}} \cdot \vec{e}_{1}, \vec{\chi}_{\rho} \cdot \vec{\tau}\right) \\
& -\left(\pi\left[\alpha\left(\varkappa_{\mathcal{S}}-\bar{\varkappa}\right)^{2}+2 \lambda+2 \beta \mathcal{A}_{\mathcal{S}} \varkappa_{\mathcal{S}}\right]-\varkappa_{\mathcal{S}} \vec{y}_{\mathcal{S}} \cdot \vec{\nu}-\left(\vec{y}_{\mathcal{S}}\right)_{s} \cdot \vec{\tau}, \vec{\chi} \cdot \vec{e}_{1}\left|\vec{x}_{\rho}\right|\right) \\
& +\left(\vec{x} \cdot \vec{e}_{1} \varkappa_{\mathcal{S}} \vec{y}_{\mathcal{S}}^{\perp}, \vec{\chi}_{\rho}\right)-\sum_{p \in \partial_{S F} I \cup \partial_{F} I}\left[\left[2 \pi \varsigma+\vec{m} \cdot \vec{y}_{\mathcal{S}}\right] \vec{\chi} \cdot \vec{e}_{1}\right](p)=\sum_{i=1}^{5} S_{i}(\vec{\chi}) \quad \forall \vec{\chi} \in \mathbb{X} .
\end{aligned}
$$

On recalling (2.3), (3.5b) and (A.3) throughout these $S_{i}(\vec{\chi})$ calculations, we have that

$$
\begin{aligned}
S_{1}(\vec{\chi}) & =\left(\vec{x} \cdot \vec{e}_{1}\left(\left(\vec{y}_{\mathcal{S}}\right)_{s} \cdot \vec{\nu}\right) \vec{\nu}, \vec{\chi}_{\rho}\right) \\
& =-\left(\left[\vec{x} \cdot \vec{e}_{1}\left(\left(\vec{y}_{\mathcal{S}}\right)_{s} \cdot \vec{\nu}\right) \vec{\nu}\right]_{s}, \vec{\chi}\left|\vec{x}_{\rho}\right|\right)+\sum_{p \in \mathcal{B}}(-1)^{p+1}\left[\vec{x} \cdot \vec{e}_{1}\left(\left(\vec{y}_{\mathcal{S}}\right)_{s} \cdot \vec{\nu}\right) \vec{\chi} \cdot \vec{\nu}\right](p)=A_{1}(\vec{\chi})+B_{1}(\vec{\chi}) .
\end{aligned}
$$

Moreover, it holds that

$$
\begin{aligned}
S_{2}(\vec{\chi})= & -\left(\left[\pi \vec{x} \cdot \vec{e}_{1}\left[\alpha\left(\varkappa_{\mathcal{S}}-\bar{\varkappa}\right)^{2}+2 \lambda+2 \beta \mathcal{A}_{\mathcal{S}} \varkappa_{\mathcal{S}}\right]-\vec{y}_{\mathcal{S}} \cdot \vec{e}_{1}\right] \vec{\tau}, \vec{\chi}_{\rho}\right) \\
= & \left(\left[\left(\pi \vec{x} \cdot \vec{e}_{1}\left[\alpha\left(\varkappa_{\mathcal{S}}-\bar{\varkappa}\right)^{2}+2 \lambda+2 \beta \mathcal{A}_{\mathcal{S}} \varkappa_{\mathcal{S}}\right]-\vec{y}_{\mathcal{S}} \cdot \vec{e}_{1}\right) \vec{\tau}\right]_{s}, \vec{\chi}\left|\vec{x}_{\rho}\right|\right) \\
& -\sum_{p \in \mathcal{B}}(-1)^{p+1}\left[\left(\pi \vec{x} \cdot \vec{e}_{1}\left[\alpha\left(\varkappa_{\mathcal{S}}-\bar{\varkappa}\right)^{2}+2 \lambda+2 \beta \mathcal{A}_{\mathcal{S}} \varkappa_{\mathcal{S}}\right]-\vec{y}_{\mathcal{S}} \cdot \vec{e}_{1}\right) \vec{\chi} \cdot \vec{\tau}\right](p)=A_{2}(\vec{\chi})+B_{2}(\vec{\chi}) .
\end{aligned}
$$

In addition,

$$
S_{3}(\vec{\chi})=-\left(\left[\pi\left[\alpha\left(\varkappa_{\mathcal{S}}-\bar{\varkappa}\right)^{2}+2 \lambda+2 \beta \mathcal{A}_{\mathcal{S}} \varkappa_{\mathcal{S}}\right]-\varkappa_{\mathcal{S}} \vec{y}_{\mathcal{S}} \cdot \vec{\nu}-\left(\vec{y}_{\mathcal{S}}\right)_{s} \cdot \vec{\tau}\right] \vec{e}_{1}, \vec{\chi}\left|\vec{x}_{\rho}\right|\right)=A_{3}(\vec{\chi}) .
$$

Finally,

$$
S_{4}(\vec{\chi})=\left(\vec{x} \cdot \vec{e}_{1} \varkappa_{\mathcal{S}} \vec{y}_{\mathcal{S}}^{\perp}, \vec{\chi}_{\rho}\right)=-\left(\left[\vec{x} \cdot \vec{e}_{1} \varkappa_{\mathcal{S}} \vec{y}_{\mathcal{S}}^{\perp}\right]_{s}, \vec{\chi}\left|\vec{x}_{\rho}\right|\right)+\sum_{p \in \mathcal{B}}(-1)^{p+1}\left[\vec{x} \cdot \vec{e}_{1} \varkappa_{\mathcal{S}} \vec{\chi} \cdot \vec{y}_{\mathcal{S}}^{\perp}\right](p)=A_{4}(\vec{\chi})+B_{4}(\vec{\chi})
$$

and we also set $B_{3}(\vec{\chi})=S_{5}(\vec{\chi})$. With the above definitions, we can write (A.20) as

$$
2 \pi\left(\left(\vec{x} \cdot \vec{e}_{1}\right) \vec{x}_{t} \cdot \vec{\nu}, \vec{\chi} \cdot \vec{\nu}\left|\vec{x}_{\rho}\right|\right)=\sum_{i=1}^{5} S_{i}(\vec{\chi})=\sum_{i=1}^{4} A_{i}(\vec{\chi})+\sum_{i=1}^{4} B_{i}(\vec{\chi}) \quad \forall \vec{\chi} \in \mathbb{X} .
$$

On recalling $(3.1 \mathrm{~b})$, we observe that

$$
A_{4}(\vec{\chi})=-\left(\left[\vec{x} \cdot \vec{e}_{1} \varkappa_{\mathcal{S}} \vec{y}_{\mathcal{S}}^{\perp}\right]_{s}, \vec{\chi}\left|\vec{x}_{\rho}\right|\right)=-\left(\left[\vec{x} \cdot \vec{e}_{1} \varkappa_{\mathcal{S}}\left(\left(\vec{y}_{\mathcal{S}} \cdot \vec{\nu}\right) \vec{\tau}-\left(\vec{y}_{\mathcal{S}} \cdot \vec{\tau}\right) \vec{\nu}\right)\right]_{s}, \vec{\chi}\left|\vec{x}_{\rho}\right|\right) .
$$

Choosing $\vec{\chi}=\chi \vec{\tau}$, for $\chi \in H_{0}^{1}(I)$, in (A.25), and combining (A.21)-(A.24) and (A.26), and recalling $\vec{\tau}_{s} \cdot \vec{\tau}=0$, (A.10), (2.3), (A.19) and (2.7), we obtain for the right hand side of (A.25)

$$
\begin{aligned}
\sum_{i=1}^{5} S_{i}(\chi \vec{\tau})= & \sum_{i=1}^{4} A_{i}(\chi \vec{\tau}) \\
= & \left(\vec{x} \cdot \vec{e}_{1} \varkappa\left(\vec{y}_{\mathcal{S}}\right)_{s} \cdot \vec{\nu}, \chi\left|\vec{x}_{\rho}\right|\right)+\left(\left[\pi \vec{x} \cdot \vec{e}_{1}\left[\alpha\left(\varkappa_{\mathcal{S}}-\bar{\varkappa}\right)^{2}+2 \lambda+2 \beta \mathcal{A}_{\mathcal{S}} \varkappa_{\mathcal{S}}\right]-\vec{y}_{\mathcal{S}} \cdot \vec{e}_{1}\right]_{\mathcal{S}}, \chi\left|\vec{x}_{\rho}\right|\right) \\
& -\left(\left[\pi\left[\alpha\left(\varkappa_{\mathcal{S}}-\bar{\varkappa}\right)^{2}+2 \lambda+2 \beta \mathcal{A}_{\mathcal{S}} \varkappa_{\mathcal{S}}\right]-\varkappa_{\mathcal{S}} \vec{y}_{\mathcal{S}} \cdot \vec{\nu}-\left(\vec{y}_{\mathcal{S}}\right)_{s} \cdot \vec{\tau}\right] \vec{\tau} \cdot \vec{e}_{1}, \chi\left|\vec{x}_{\rho}\right|\right)
\end{aligned}
$$




$$
\begin{aligned}
& -\left(\vec{x} \cdot \vec{e}_{1} \varkappa_{\mathcal{S}}\left[\left(\vec{y}_{\mathcal{S}} \cdot \vec{\nu}\right)_{s}+\varkappa \vec{y}_{\mathcal{S}} \cdot \vec{\tau}\right]+\left(\vec{x} \cdot \vec{e}_{1} \varkappa_{\mathcal{S}}\right)_{s} \vec{y}_{\mathcal{S}} \cdot \vec{\nu}, \chi\left|\vec{x}_{\rho}\right|\right) \\
= & \left(\vec{x} \cdot \vec{e}_{1}\left(\varkappa-\varkappa_{\mathcal{S}}\right)\left(\vec{y}_{\mathcal{S}}\right)_{s} \cdot \vec{\nu}, \chi\left|\vec{x}_{\rho}\right|\right)+\left(\vec{x} \cdot \vec{e}_{1}\left(\pi\left[\alpha\left(\varkappa_{\mathcal{S}}-\bar{\varkappa}\right)^{2}+2 \beta \mathcal{A}_{\mathcal{S}} \varkappa_{\mathcal{S}}\right]_{s}-\left(\varkappa_{\mathcal{S}}\right)_{s} \vec{y}_{\mathcal{S}} \cdot \vec{\nu}\right), \chi\left|\vec{x}_{\rho}\right|\right) \\
& -\left(\left(\vec{y}_{\mathcal{S}}\right)_{s} \cdot\left(\vec{e}_{1}-\left(\vec{\tau} \cdot \vec{e}_{1}\right) \vec{\tau}\right), \chi\left|\vec{x}_{\rho}\right|\right) \\
= & 0
\end{aligned}
$$

as expected.

Choosing $\vec{\chi}=\chi \vec{\nu}$, for $\chi \in H_{0}^{1}(I)$, in (A.25), and combining (A.21)-(A.24) and (A.26), and on recalling (2.6), $\vec{\nu}_{s} \cdot \vec{\nu}=0,($ A.19) and (2.7), we obtain for the right hand side of (A.25)

$$
\begin{aligned}
& \sum_{i=1}^{5} S_{i}(\chi \vec{\nu})=\sum_{i=1}^{4} A_{i}(\chi \vec{\nu})=-\left(\left[\vec{x} \cdot \vec{e}_{1}\left(\left(\vec{y}_{\mathcal{S}}\right)_{s} \cdot \vec{\nu}\right)\right]_{s}, \chi\left|\vec{x}_{\rho}\right|\right) \\
& +\left(\left[\pi \vec{x} \cdot \vec{e}_{1}\left[\alpha\left(\varkappa_{\mathcal{S}}-\bar{\varkappa}\right)^{2}+2 \lambda+2 \beta \mathcal{A}_{\mathcal{S}} \varkappa_{\mathcal{S}}\right]-\vec{y}_{\mathcal{S}} \cdot \vec{e}_{1}\right] \varkappa, \chi\left|\vec{x}_{\rho}\right|\right) \\
& -\left(\left[\pi\left[\alpha\left(\varkappa_{\mathcal{S}}-\bar{\varkappa}\right)^{2}+2 \lambda+2 \beta \mathcal{A}_{\mathcal{S}} \varkappa_{\mathcal{S}}\right]-\varkappa_{\mathcal{S}} \vec{y}_{\mathcal{S}} \cdot \vec{\nu}-\left(\vec{y}_{\mathcal{S}}\right)_{s} \cdot \vec{\tau}\right] \vec{\nu} \cdot \vec{e}_{1}, \chi\left|\vec{x}_{\rho}\right|\right) \\
& -\left(\vec{x} \cdot \vec{e}_{1} \varkappa_{\mathcal{S}} \vec{y}_{\mathcal{S}} \cdot \vec{\nu}-\left[\vec{x} \cdot \vec{e}_{1} \varkappa_{\mathcal{S}} \vec{y}_{\mathcal{S}} \cdot \vec{\tau}\right]_{S}, \chi\left|\vec{x}_{\rho}\right|\right) \\
& =-\left(\left[\vec{x} \cdot \vec{e}_{1}\left(\left(\vec{y}_{\mathcal{S}} \cdot \vec{\nu}\right)_{s}+\left(\varkappa-\varkappa_{\mathcal{S}}\right) \vec{y}_{\mathcal{S}} \cdot \vec{\tau}\right)\right]_{S}, \chi\left|\vec{x}_{\rho}\right|\right) \\
& +\left(\left[\pi \vec{x} \cdot \vec{e}_{1}\left[\alpha\left(\varkappa_{\mathcal{S}}-\bar{\varkappa}\right)^{2}+2 \lambda+2 \beta \mathcal{A}_{\mathcal{S}} \varkappa_{\mathcal{S}}\right]-\vec{y}_{\mathcal{S}} \cdot \vec{e}_{1}\right] \varkappa, \chi\left|\vec{x}_{\rho}\right|\right) \\
& -\left(\left[\pi\left[\alpha\left(\varkappa_{\mathcal{S}}-\bar{\varkappa}\right)^{2}+2 \lambda+2 \beta \mathcal{A}_{\mathcal{S}} \varkappa_{\mathcal{S}}\right]+\left(\varkappa-\varkappa_{\mathcal{S}}\right) \vec{y}_{\mathcal{S}} \cdot \vec{\nu}-\left(\vec{y}_{\mathcal{S}} \cdot \vec{\tau}\right)_{S}\right] \vec{\nu} \cdot \vec{e}_{1}, \chi\left|\vec{x}_{\rho}\right|\right) \\
& -\left(\vec{x} \cdot \vec{e}_{1} \varkappa \varkappa_{\mathcal{S}} \vec{y}_{\mathcal{S}} \cdot \vec{\nu}, \chi\left|\vec{x}_{\rho}\right|\right) \\
& =-2 \pi \alpha\left(\left[\vec{x} \cdot \vec{e}_{1}\left(\varkappa_{\mathcal{S}}\right)_{s}\right]_{s}, \chi\left|\vec{x}_{\rho}\right|\right)-\left(\left[\vec{x} \cdot \vec{e}_{1}\left(\varkappa-\varkappa_{\mathcal{S}}\right) \vec{y}_{\mathcal{S}} \cdot \vec{\tau}\right]_{s}, \chi\left|\vec{x}_{\rho}\right|\right) \\
& +\left(\pi \vec{x} \cdot \vec{e}_{1}\left[\alpha\left(\varkappa_{\mathcal{S}}-\bar{\varkappa}\right)^{2}+2 \lambda+2 \beta \mathcal{A}_{\mathcal{S}} \varkappa_{\mathcal{S}}\right] \varkappa_{\mathcal{S}}, \chi\left|\vec{x}_{\rho}\right|\right)-\left(\vec{x} \cdot \vec{e}_{1}\left[\left(\varkappa-\varkappa_{\mathcal{S}}\right)^{2}+\varkappa_{\mathcal{S}}\right] \vec{y}_{\mathcal{S}} \cdot \vec{\nu}, \chi\left|\vec{x}_{\rho}\right|\right) \\
& -\left(\vec{y}_{\mathcal{S}} \cdot \vec{e}_{1} \varkappa-\left(\vec{y}_{\mathcal{S}} \cdot \vec{\tau}\right)_{s} \vec{x} \cdot \vec{e}_{1}\left(\varkappa-\varkappa_{\mathcal{S}}\right), \chi\left|\vec{x}_{\rho}\right|\right) \\
& =-2 \pi \alpha\left(\left[\vec{x} \cdot \vec{e}_{1}\left(\varkappa_{\mathcal{S}}\right)_{s}\right]_{s}, \chi\left|\vec{x}_{\rho}\right|\right)-\left(\left[\vec{x} \cdot \vec{e}_{1}\left(\varkappa-\varkappa_{\mathcal{S}}\right)\right]_{s} \vec{y}_{\mathcal{S}} \cdot \vec{\tau}, \chi\left|\vec{x}_{\rho}\right|\right) \\
& -\pi \alpha\left(\vec{x} \cdot \vec{e}_{1}\left[\left(\varkappa_{\mathcal{S}}\right)^{2}+\bar{\varkappa} \varkappa_{\mathcal{S}}+2 \varkappa^{2}-2 \varkappa_{\mathcal{S}}\right]\left(\varkappa_{\mathcal{S}}-\bar{\varkappa}\right), \chi\left|\vec{x}_{\rho}\right|\right) \\
& -\left(\vec{y}_{\mathcal{S}} \cdot \vec{e}_{1} \varkappa, \chi\left|\vec{x}_{\rho}\right|\right)+2 \pi \lambda\left(\vec{x} \cdot \vec{e}_{1} \varkappa_{\mathcal{S}}, \chi\left|\vec{x}_{\rho}\right|\right)-2 \pi \beta \mathcal{A}_{\mathcal{S}}\left(\varkappa \vec{x} \cdot \vec{e}_{1}\left(\varkappa-\varkappa_{\mathcal{S}}\right), \chi\left|\vec{x}_{\rho}\right|\right) \\
& =-2 \pi \alpha\left(\left[\vec{x} \cdot \vec{e}_{1}\left(\varkappa_{\mathcal{S}}\right)_{s}\right]_{S}, \chi\left|\vec{x}_{\rho}\right|\right)-\left(\left[\vec{x} \cdot \vec{e}_{1}\left(\varkappa-\varkappa_{\mathcal{S}}\right)\right]_{s} \vec{y}_{\mathcal{S}} \cdot \vec{\tau}, \chi\left|\vec{x}_{\rho}\right|\right) \\
& -\left(\pi \vec{x} \cdot \vec{e}_{1}\left[\alpha\left(\varkappa_{\mathcal{S}}^{2}-\bar{\varkappa}^{2}\right)-2 \lambda\right] \varkappa_{\mathcal{S}}+\vec{y}_{\mathcal{S}} \cdot \vec{e}_{1} \varkappa, \chi\left|\vec{x}_{\rho}\right|\right)+2 \pi\left(\vec { x } \cdot \vec { e } _ { 1 } \left[\alpha\left(\varkappa_{\mathcal{S}}-\bar{\varkappa}\right)\right.\right. \\
& \left.\left.+\beta \mathcal{A}_{\mathcal{S}}\right]\left(\varkappa_{\mathcal{S}}-\varkappa\right) \varkappa, \chi\left|\vec{x}_{\rho}\right|\right) \text {. }
\end{aligned}
$$

The desired result (2.24) follows from (A.20) and (A.27) on noting that

$$
\begin{gathered}
-\left[\vec{x} \cdot \vec{e}_{1}\left(\varkappa-\varkappa_{\mathcal{S}}\right)\right]_{\mathcal{S}} \vec{y}_{\mathcal{S}} \cdot \vec{\tau}-\vec{y}_{\mathcal{S}} \cdot \vec{e}_{1} \varkappa=-\left(\vec{\nu}_{S} \cdot \vec{e}_{1}\right) \vec{y}_{\mathcal{S}} \cdot \vec{\tau}-\vec{y}_{\mathcal{S}} \cdot \vec{e}_{1} \varkappa=-\left(\vec{y}_{\mathcal{S}} \cdot \vec{\nu}\right) \vec{\nu} \cdot \vec{e}_{1} \varkappa \\
=2 \pi \vec{x} \cdot \vec{e}_{1}\left[\alpha\left(\varkappa_{\mathcal{S}}-\bar{\varkappa}\right)+\beta \mathcal{A}_{\mathcal{S}}\right](\varkappa \mathcal{S}-\varkappa) \varkappa=2 \pi \vec{x} \cdot \vec{e}_{1}\left[\alpha\left(\varkappa_{\mathcal{S}}-\bar{\varkappa}\right)+\beta \mathcal{A}_{\mathcal{S}}\right] \mathcal{K}_{\mathcal{S}},
\end{gathered}
$$

where we have recalled (2.7), (A.10) and (A.19).

We now need to show that the weak formulation (3.44) enforces the claimed boundary conditions. As in Section A.1, the conditions (2.31a), (2.33a), (2.34a) and (2.35a) are enforces strongly. We also notice that (3.44c) enforces (2.35b) and (2.33b), recall the discussion below (3.9). This means that we still need to show (2.35c), (2.30), (2.31b), (2.31c) and (2.34b).

In order to show (2.35c) we argue similarly to Section A.1. Choosing $\vec{\chi}=\chi \vec{\nu}$ in (A.20), where $\chi \in H^{1}(I)$ is zero away from $\partial_{0} I$, that the term $2 \pi \alpha\left(\vec{x} \cdot \vec{e}_{1}\left(\varkappa_{\mathcal{S}}\right)_{\rho}, \chi_{\rho}\left|\vec{x}_{\rho}\right|^{-1}\right)$ equals a sum of inner products that have integrands that converge to zero for $\rho$ approaching $\partial_{0} I$. We can then again use Appendix A.1 of [7] to show that (3.44a) implies the boundary condition (2.35c). 
Next we recall from (2.37) and (3.37) that

$$
\left(\vec{x} \cdot \vec{e}_{1}\right) \vec{y}_{\mathcal{S}}=2 \pi \alpha_{G} \vec{e}_{1} \quad \text { on } \quad \partial_{M} I=\partial_{N} I \cup \partial_{S F} I \cup \partial_{F} I
$$

Combining with (A.19) and noting (2.2a) yields (A.16). Hence, we have that (2.30c), (2.31c) and (2.34b) are imposed strongly. Overall, we still need to show (2.30a), (2.30b) and (2.31b). It follows from (A.21) and (A.24), on recalling (A.10), (3.1b), (A.19), (2.7) and (2.2b), that

$$
\begin{aligned}
B_{1}(\vec{\chi})+B_{4}(\vec{\chi})= & \sum_{p \in \mathcal{B}}(-1)^{p+1}\left[\left(\vec{x} \cdot \vec{e}_{1}\right)\left(\left(\left(\vec{y}_{\mathcal{S}}\right)_{s} \cdot \vec{\nu}\right) \vec{\nu}+\varkappa_{\mathcal{S}} \vec{y}_{\mathcal{S}}^{\perp}\right) \cdot \vec{\chi}\right](p) \\
= & \sum_{p \in \mathcal{B}}(-1)^{p+1}\left[\left(\vec{x} \cdot \vec{e}_{1}\right)\left(\left[\left(\vec{y}_{\mathcal{S}} \cdot \vec{\nu}\right)_{s}+\left(\varkappa-\varkappa_{\mathcal{S}}\right)\left(\vec{y}_{\mathcal{S}} \cdot \vec{\tau}\right)\right] \vec{\nu}+\varkappa_{\mathcal{S}}\left(\vec{y}_{\mathcal{S}} \cdot \vec{\nu}\right) \vec{\tau}\right) \cdot \vec{\chi}\right](p) \\
= & \sum_{p \in \mathcal{B}}(-1)^{p+1}\left[\left[2 \pi \alpha\left(\vec{x} \cdot \vec{e}_{1}\right)\left(\kappa_{\mathcal{S}}\right)_{s}+\left(\vec{\nu} \cdot \vec{e}_{1}\right)\left(\vec{y}_{\mathcal{S}} \cdot \vec{\tau}\right)\right] \vec{\nu} \cdot \vec{\chi}\right](p) \\
& +\sum_{p \in \partial_{S F} I \cup \partial_{F} I}(-1)^{p+1}\left[\left(\vec{x} \cdot \vec{e}_{1}\right) \varkappa_{\mathcal{S}}\left(\vec{y}_{\mathcal{S}} \cdot \vec{\nu}\right) \vec{\tau} \cdot \vec{\chi}\right](p) .
\end{aligned}
$$

Looking first at the normal boundary contributions, we compute, on noting that $\vec{m}=\vec{\mu}$ and (2.20),

$$
\begin{aligned}
\sum_{i=1}^{4} B_{i}(\chi \vec{\nu}) & =\sum_{p \in \mathcal{B}}(-1)^{p+1}\left[\left[2 \pi \alpha\left(\vec{x} \cdot \vec{e}_{1}\right)\left(\kappa_{\mathcal{S}}\right)_{s}+\left(\vec{\nu} \cdot \vec{e}_{1}\right)(\vec{y} \mathcal{S} \cdot \vec{\tau})\right] \chi\right](p)-\sum_{p \in \partial_{S F} I \cup \partial_{F} I}\left[\left[2 \pi \varsigma+\vec{m} \cdot \vec{y}_{\mathcal{S}}\right]\left(\vec{\nu} \cdot \vec{e}_{1}\right) \chi\right](p) \\
& =2 \pi \alpha \sum_{p \in \mathcal{B}}(-1)^{p+1}\left[\vec{x} \cdot \vec{e}_{1}\left(\varkappa_{\mathcal{S}}\right)_{s} \chi\right](p)-2 \pi \varsigma \sum_{p \in \partial_{S F} I \cup \partial_{F} I}\left[\vec{x} \cdot \vec{e}_{1} \frac{\vec{\nu} \cdot \vec{e}_{1}}{\vec{x} \cdot \vec{e}_{1}} \chi\right](p) \\
& =2 \pi \sum_{p \in \partial_{S F} I \cup \partial_{F} I}\left[\vec{x} \cdot \vec{e}_{1}\left((-1)^{p+1} \alpha\left(\varkappa_{\mathcal{S}}\right)_{s}-\varsigma \frac{\vec{\nu} \cdot \vec{e}_{1}}{\vec{x} \cdot \vec{e}_{1}}\right) \chi\right](p) \quad \forall \chi \in H^{1}(I) .
\end{aligned}
$$

where in the last step we have observed (2.35c) and (A.3). This gives (2.30a) on $\partial_{F} I$ on recalling (2.2a) and (3.5b).

Next we consider the tangential components. It holds, on noting that $\vec{m}=\vec{\mu},(2.20),(2.7)$ and (A.28), that

$$
\begin{aligned}
\sum_{i=1}^{4} B_{i}(\chi \vec{\tau})= & \sum_{p \in \partial_{S F} I \cup \partial_{F} I}(-1)^{p+1}\left[\left(\vec{x} \cdot \vec{e}_{1}\right) \varkappa_{\mathcal{S}}\left(\vec{y}_{\mathcal{S}} \cdot \vec{\nu}\right) \chi\right](p) \\
& -\sum_{p \in \partial_{S F} I \cup \partial_{F} I}(-1)^{p+1}\left[\left(\pi \vec{x} \cdot \vec{e}_{1}\left[\alpha\left(\varkappa_{\mathcal{S}}-\bar{\varkappa}\right)^{2}+2 \lambda+2 \beta \mathcal{A}_{\mathcal{S}} \varkappa_{\mathcal{S}}\right]-\vec{y}_{\mathcal{S}} \cdot \vec{e}_{1}\right) \chi\right](p) \\
& -2 \pi \varsigma \sum_{p \in \partial_{S F} I \cup \partial_{F} I}\left[\left(\vec{\tau} \cdot \vec{e}_{1}\right) \chi\right](p)-\sum_{p \in \partial_{S F} I \cup \partial_{F} I}(-1)^{p+1}\left[\left(\vec{y}_{\mathcal{S}} \cdot \vec{\tau}\right)\left(\vec{\tau} \cdot \vec{e}_{1}\right) \chi\right](p) \\
= & \sum_{p \in \partial_{S F} I \cup \partial_{F} I}(-1)^{p+1}\left[\left(\vec{x} \cdot \vec{e}_{1}\right) \varkappa\left(\vec{y}_{\mathcal{S}} \cdot \vec{\nu}\right) \chi\right](p)-2 \pi \varsigma \sum_{p \in \partial_{S F} I \cup \partial_{F} I}\left[\left(\vec{\tau} \cdot \vec{e}_{1}\right) \chi\right](p) \\
& -\sum_{p \in \partial_{S F} I \cup \partial_{F} I}(-1)^{p+1}\left[\pi \vec{x} \cdot \vec{e}_{1}\left[\alpha\left(\varkappa_{\mathcal{S}}-\bar{\varkappa}\right)^{2}+2 \lambda+2 \beta \mathcal{A}_{\mathcal{S}} \varkappa_{\mathcal{S}}\right] \chi\right](p)
\end{aligned}
$$




$$
\begin{aligned}
= & -2 \pi \alpha_{G} \sum_{p \in \partial_{S F} I \cup \partial_{F} I}(-1)^{p+1}\left[\left(\vec{x} \cdot \vec{e}_{1}\right) \mathcal{K}_{\mathcal{S}} \chi\right](p) \\
& -\sum_{p \in \partial_{S F} I \cup \partial_{F} I}(-1)^{p+1}\left[\pi \vec{x} \cdot \vec{e}_{1}\left[\alpha\left(\varkappa_{\mathcal{S}}-\bar{\varkappa}\right)^{2}+2 \lambda+2 \beta \mathcal{A}_{\mathcal{S}} \varkappa_{\mathcal{S}}\right] \chi\right](p) \\
& -2 \pi \varsigma \sum_{p \in \partial_{S F} I \cup \partial_{F} I}(-1)^{p+1}\left[\vec{x} \cdot \vec{e}_{1} \frac{\vec{\mu} \cdot \vec{e}_{1}}{\vec{x} \cdot \vec{e}_{1}} \chi\right](p) \quad \forall \chi \in H^{1}(I) .
\end{aligned}
$$

This yields (2.30b) on $\partial_{F} I$ on recalling (2.2a). In order to prove (2.31b) on $\partial_{S F} I$, we choose a test function $\vec{\chi} \in \mathbb{X}$ and then combine (A.29) and (A.30), similarly to the previous section, Section A.1.

\section{Appendix B. Singularities for Willmore flow of Genus-1 Surfaces}

In the recent paper [17] it was shown that a torus of revolution $\mathcal{S}(0)$, with profile curve $\Gamma(0)$ such that its turning number $\mathrm{T}(\Gamma)=\frac{1}{2 \pi} \int_{I} \varkappa\left|\vec{x}_{\rho}\right| \mathrm{d} \rho$ is zero, will develop a singularity under Willmore flow. Here we recall that for an immersed curve $\Gamma \subset \mathbb{R}^{2}, T(\Gamma) \in \mathbb{Z}$ is the winding number with respect to the origin of the tangent vector $\vec{\tau}$. In particular, it is shown in Lemma 4.8 of [17] that either a singularity will develop in finite time, or as $t \rightarrow \infty$ one of the following quantities will grow unbounded: $a(t)=\sup _{I}\left[\varkappa^{2}+\left(\frac{\vec{\nu} \cdot \vec{e}_{1}}{\vec{x} \cdot \vec{e}_{1}}\right)^{2}\right]^{\frac{1}{2}}$ or $|\Gamma(t)|_{\mathbb{H}^{2}}=\left(\left(\vec{x} \cdot \vec{e}_{1}\right)^{-1},\left|\vec{x}_{\rho}\right|\right)$, where $a(t)$ is a suitable $L^{\infty}$-norm of the second fundamental form on $\mathcal{S}(t)$, and where $|\Gamma(t)|_{\mathbb{H}^{2}}$ denotes the length of the curve $\Gamma(t)$ in the hyperbolic plane $\mathbb{H}^{2}$, see e.g. (2.6a) of [10].

In this appendix, we will present numerical evidence that $T(\Gamma)=0$ appears to be a necessary condition for a singularity to develop, and that any such singularity is only attained as $t \rightarrow \infty$. To this end, we consider the case $\partial I=\emptyset$ from now on and define

$$
\left|\Gamma^{m}\right|_{\mathbb{H}^{2}, h}=\left(\left(\vec{X}^{m} \cdot \vec{e}_{1}\right)^{-1},\left|\vec{X}_{\rho}^{m}\right|\right)^{h}
$$

for $\Gamma^{m}=\vec{X}^{m}(I)$ and $\vec{X}^{m} \in \underline{V}^{h}$, as a discrete analogue to $|\Gamma(t)|_{\mathbb{H}^{2}}$. We remark that a corresponding discrete analogue of $a(t)$ in practice behaved very similarly to (B.1), and so we omit its discussion here.

We begin with two experiments for the case $T(\Gamma) \neq 0$. In particular, we choose as initial data a generating curve that is made up of a circle of radius 1 centered at $\sqrt{2} \vec{e}_{1}$ and a circle of radius $r$ centered at $(\sqrt{2} \pm(1-r)) \vec{e}_{1}$, with $r=0.1$, so that $\mathrm{T}(\Gamma)= \pm 2$. The simulations of Willmore flow for the surfaces generated by these curves is shown in Figure B.1. The discretization parameters for the scheme $\left(\mathcal{P}^{m}\right)^{h}$ are $J=512$ and $\Delta t=10^{-4}$. When the smaller circle is inscribed on the right, then the evolution immediately approaches a double-covering of the Clifford torus, recall also Figure 2. If the smaller circle is inscribed on the left, a more complicated evolution ensues, but eventually a double covering of the Clifford torus is reached. At time $t=100$, both evolutions have reached a discrete Willmore energy of $78.96 \approx 8 \pi^{2}$, i.e. about twice the Willmore energy of the Clifford torus.

Overall the results in Figure B.1 indicate that $T(\Gamma)=0$ is a necessary condition for a singularity under Willmore flow to occur.

We now concentrate on the possible onset of a singularity. To this end, we show the evolution for two initial lemniscates in Figures B.2 and B.3. The discretization parameters for the scheme $\left(\mathcal{P}^{m}\right)^{h}$ are $J=1024$ and $\Delta t=10^{-6}$. The initial data satisfy $\mathrm{T}(\Gamma)=0$, and so the result in Lemma 4.8 of [17] yields that Willmore flow will develop a singularity either in finite time or as $t \rightarrow \infty$. In the first experiment the part of the lemniscate with larger radius is close to the $x_{2}$-axis. But during the evolution that part thins and approaches the axis. In the second experiment, the initial lemniscate is rotated by 180 degrees, so that the part with the smaller radius is close to the $x_{2}$-axis. During the evolution that parts thins even more and approaches the axis.

To investigate the behaviour close to the $x_{2}$-axis further, and to help decide whether the evolution reaches a singularity in finite time, we start a refined computation from the solution at time $t=0.3$ in Figure B.3. The results for $J=4096$ and $\Delta t=10^{-8}$ are shown in Figure B.4. The discrete quantity (B.1), which we plot on the right of Figure B.4, appears to grow polynomial in time. In particular, it seems to grow only slightly 

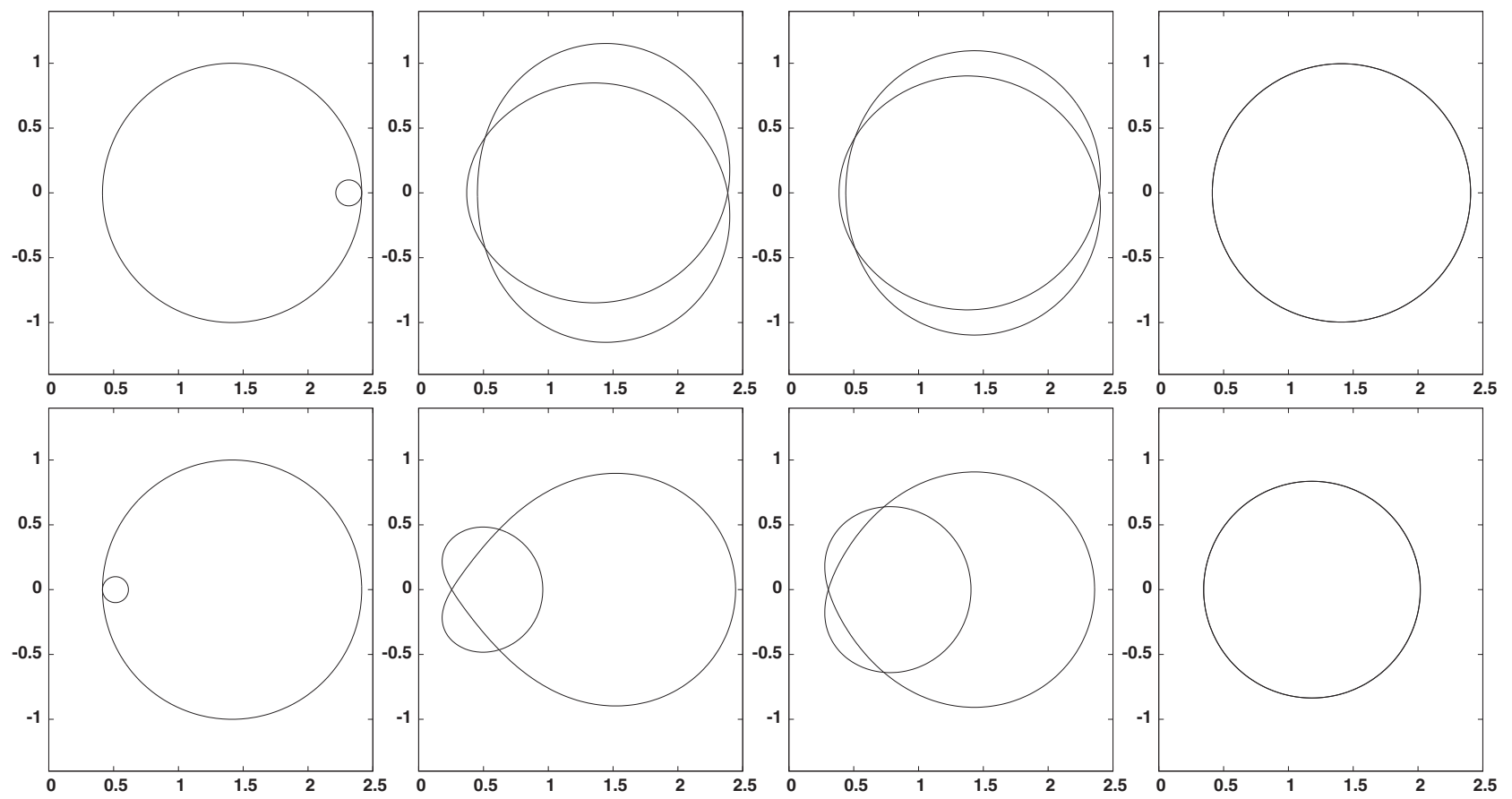

Figure B.1. $\left(\mathcal{P}^{m}\right)^{h}$ Willmore flow for $\mathrm{T}(\Gamma)= \pm 2$. We show the two evolutions at times $t=0,1,5,100$.
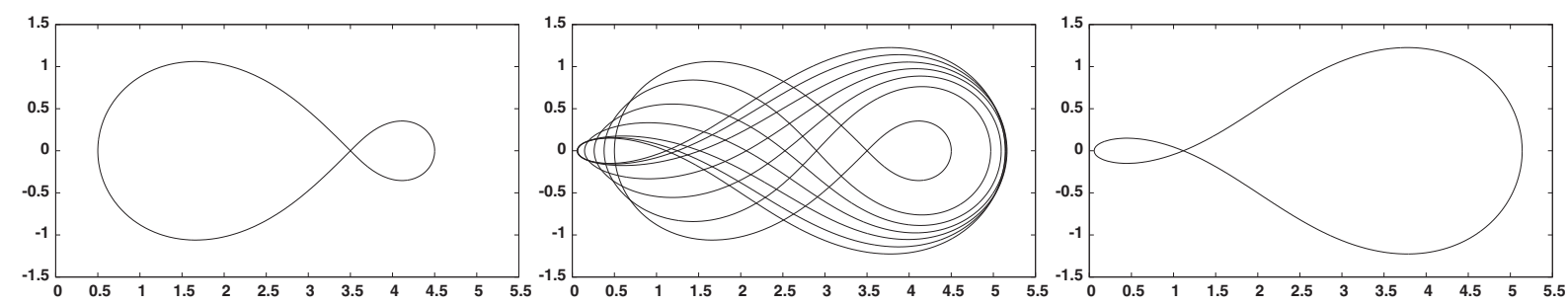

Figure B.2. $\left(\mathcal{P}^{m}\right)^{h}$ Willmore flow for $\mathrm{T}(\Gamma)=0$. We show the solution at time $t=0$, at times times $t=0,0.5, \ldots, 3$ and at time $t=3$.
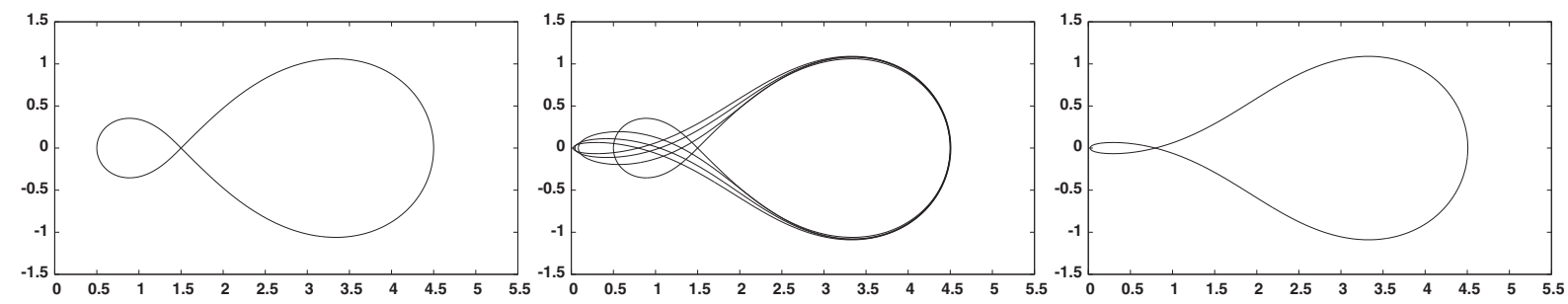

FiguRE B.3. $\left(\mathcal{P}^{m}\right)^{h}$ Willmore flow for $\mathrm{T}(\Gamma)=0$. We show the solution at time $t=0$, at times times $t=0,0.1, \ldots, 0.3$ and at time $t=0.3$. 

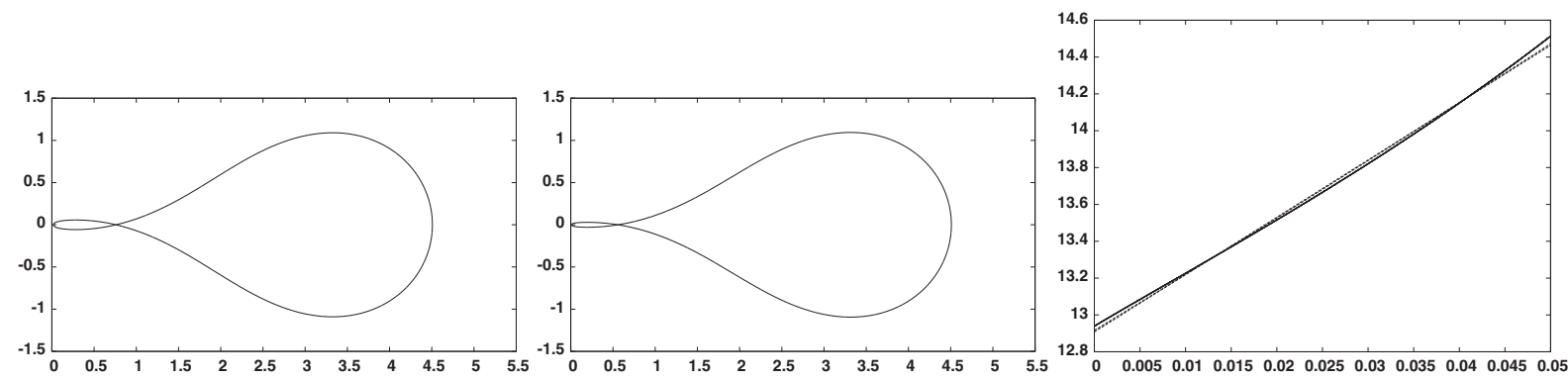

Figure B.4. $\left(\mathcal{P}^{m}\right)^{h}$ Willmore flow for $\mathrm{T}(\Gamma)=0$. The solution at times $t=0,0.05$. On the right a plot of $\left|\Gamma^{m}\right|_{\mathbb{H}^{2}, h}$, recall (B.1), over time, together with the best fits $f(t)=a(1+t)^{b}$ and $g(t)=c+d t$, with $(a, b, c, d)=(12.9,2.3,12.9,31.1)$.
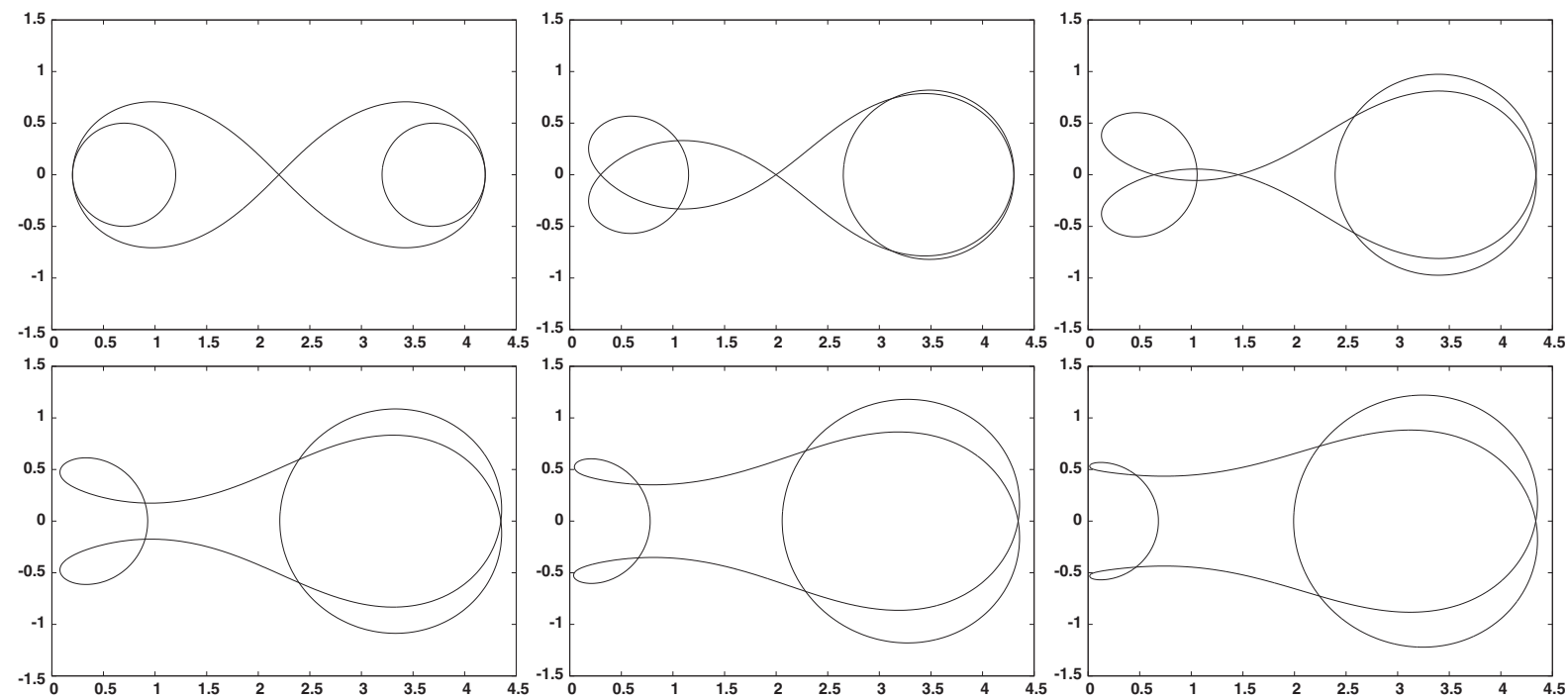

FiguRE B.5. $\left(\mathcal{P}^{m}\right)^{h}$ Willmore flow for $\mathrm{T}(\Gamma)=0$. We show the solution at times $t=$ $0,0.2, \ldots, 0.8$ and 0.9 . Here the evolution on $[0.8,0.9]$ was computed with the finer parameters $J=4096$ and $\Delta t=10^{-7}$.

faster than the best fits of the form $f(t)=a(1+t)^{b}$ and $g(t)=c+d t$, where for the best fits we observe $(a, b, c, d)=(12.9,2.3,12.9,31.1)$. It is therefore difficult to draw definite conclusions. Hence we conjecture that the singularity, where the toroidal surface closes up at the origin, is reached only as $t \rightarrow \infty$. This conjecture is in agreement with similar conclusion drawn by the authors for the onset of a singularity for a genus-0 surface that converges to two touching spheres. In particular, we stress that the evolution shown in Figure 24 in [7] closely matches the shape of the curves in Figure B.4 near the $x_{2}$-axis.

Finally, we also show a more interesting evolution for another initial data with $T(\Gamma)=0$. Here a symmetric lemniscate is inscribed with a circle on each side. The Willmore flow for a torus with such a generating curve can be seen in Figure B.5. The discretization parameters are $J=1024$ and $\Delta t=10^{-5}$, and for the final evolution on the time interval $[0.8,0.9]$ we use the finer parameters $J=4096$ and $\Delta t=10^{-7}$. We see that the circle inscribed on the right of the lemniscate grows, while the circle on the left untangles to create two loops close to the $x_{2}$-axis with large curvature. 
The discrete quantity (B.1) once again appears to grow slightly faster than the best polynomial fits, with our best fits for the final evolution in Figure B.5 given by $f(0.8+t)=a(1+t)^{b}$ and $g(0.8+t)=c+d t$, with $(a, b, c, d)=(22,1.5,22,33)$.

Overall, based on our numerical evidence, we conjecture the following: If $\mathrm{T}(\Gamma(0))=\alpha \neq 0$, then Willmore flow will converge to a $|\alpha|$-covering of the Clifford torus. If $\mathrm{T}(\Gamma(0))=0$, then Willmore flow will develop a singularity/blow-up as $t \rightarrow \infty$.

Acknowledgements. The authors gratefully acknowledge the support of the Regensburger Universitätsstiftung Hans Vielberth.

\section{REFERENCES}

[1] J.W. Barrett, H. Garcke and R. Nürnberg, A parametric finite element method for fourth order geometric evolution equations. J. Comput. Phys. 222 (2007) 441-462.

[2] J.W. Barrett, H. Garcke and R. Nürnberg, Parametric approximation of Willmore flow and related geometric evolution equations. SIAM J. Sci. Comput. 31 (2008) 225-253.

[3] J.W. Barrett, H. Garcke and R. Nürnberg, Elastic flow with junctions: Variational approximation and applications to nonlinear splines. Math. Models Methods Appl. Sci. 22 (2012) 1250037.

[4] J.W. Barrett, H. Garcke and R. Nürnberg, Parametric approximation of isotropic and anisotropic elastic flow for closed and open curves. Numer. Math. 120 (2012) 489-542.

[5] J.W. Barrett, H. Garcke and R. Nürnberg, Computational parametric Willmore flow with spontaneous curvature and area difference elasticity effects. SIAM J. Numer. Anal. 54 (2016) 1732-1762.

[6] J.W. Barrett, H. Garcke and R. Nürnberg, Stable variational approximations of boundary value problems for Willmore flow with Gaussian curvature. IMA J. Numer. Anal. 37 (2017) 1657-1709.

[7] J.W. Barrett, H. Garcke and R. Nürnberg, Finite element methods for fourth order axisymmetric geometric evolution equations. J. Comput. Phys. 376 (2019) 733-766.

[8] J.W. Barrett, H. Garcke and R. Nürnberg, Stable discretizations of elastic flow in Riemannian manifolds. SIAM J. Numer. Anal. 57 (2019) 1987-2018.

[9] J.W. Barrett, H. Garcke and R. Nürnberg, Variational discretization of axisymmetric curvature flows. Numer. Math. 141 (2019) 791-837.

[10] J.W. Barrett, H. Garcke and R. Nürnberg, Numerical approximation of curve evolutions in Riemannian manifolds. IMA J. Numer. Anal. 40 (2020) 1601-1651.

[11] J.W. Barrett, H. Garcke and R. Nürnberg, Parametric finite element approximations of curvature driven interface evolutions, edited by A. Bonito and R.H. Nochetto. In: Vol. 21 of Handb. Numer. Anal. Elsevier, Amsterdam (2020) 275-423.

[12] A.I. Bobenko and P. Schröder, Discrete Willmore flow, edited by J. Fujii. In: ACM SIGGRAPH 2005 Courses. ACM, New York, NY, SIGGRAPH '05, 5-es.

[13] P.B. Canham, The minimum energy of bending as a possible explanation of the biconcave shape of the human red blood cell. J. Theor. Biol. 26 (1970) 61-81.

[14] R. Capovilla, J. Guven and J.A. Santiago, Lipid membranes with an edge. Phys. Rev. E 66 (2002) 021607.

[15] U. Clarenz, U. Diewald, G. Dziuk, M. Rumpf and R. Rusu, A finite element method for surface restoration with smooth boundary conditions. Comput. Aided Geom. Design 21 (2004) 427-445.

[16] G. Cox and J. Lowengrub, The effect of spontaneous curvature on a two-phase vesicle. Nonlinearity 28 (2015) $773-793$.

[17] A. Dall'Acqua, M. Müller, R. Schätzle and A. Spener, The Willmore flow of tori of revolution. Preprint arXiv:2005.13500 (2020).

[18] T.A. Davis, Algorithm 832: UMFPACK V4.3 - an unsymmetric-pattern multifrontal method. ACM Trans. Math. Software 30 (2004) 196-199.

[19] K. Deckelnick and G. Dziuk, Error analysis for the elastic flow of parametrized curves. Math. Comput. 78 (2009) 645-671.

[20] K. Deckelnick and F. Schieweck, Error analysis for the approximation of axisymmetric Willmore flow by $C^{1}$-finite elements. Interfaces Free Bound. 12 (2010) 551-574.

[21] G. Dziuk, Computational parametric Willmore flow. Numer. Math. 111 (2008) 55-80.

[22] S. Germain, Recherches sur la théorie des surfaces élastiques. Veuve Courcier, Paris (1821).

[23] W. Helfrich, Elastic properties of lipid bilayers: theory and possible experiments. Z. Naturforsch C 28 (1973) $693-703$.

[24] F. Jülicher and R. Lipowsky, Shape transformations of vesicles with intramembrane domains. Phys. Rev. E 53 (1996) $2670-2683$.

[25] F. Jülicher and U. Seifert, Shape equations for axisymmetric vesicles: A clarification. Phys. Rev. E 49 (1994) $4728-4731$.

[26] G.R. Kirchhoff, Über das Gleichgewicht und die Bewegung einer elastischen Scheibe. J. Reine Angew. Math. 40 (1850) 51-88.

[27] W. Kühnel, In: Vol. 77 of Differential geometry: Curves - Surfaces - Manifolds. Student Mathematical Library. Amer. Math. Soc. Providence, RI (2015). 
[28] F.C. Marques and A. Neves, Min-max theory and the Willmore conjecture. Ann. Math. 179 (2014) 683-782.

[29] U.F. Mayer and G. Simonett, A numerical scheme for axisymmetric solutions of curvature-driven free boundary problems, with applications to the Willmore flow. Interfaces Free Bound. 4 (2002) 89-109.

[30] J.C.C. Nitsche, Boundary value problems for variational integrals involving surface curvatures. Quart. Appl. Math. 51 (1993) 363-387.

[31] S.D. Poisson, Mémoire sur les surfaces élastiques. Mémoires de l'Institut 1812 9 (1814) 167-226.

[32] R.E. Rusu, An algorithm for the elastic flow of surfaces. Interfaces Free Bound. 7 (2005) 229-239.

[33] U. Seifert, Configurations of fluid membranes and vesicles. Adv. Phys. 46 (1997) 13-137.

[34] Z.C. Tu and Z.C. Ou-Yang, Lipid membranes with free edges. Phys. Rev. E 68 (2003) 061915.

[35] X. Wang and Q. Du, Modelling and simulations of multi-component lipid membranes and open membranes via diffuse interface approaches. J. Math. Biol. 56 (2008) 347-371.

[36] T.J. Willmore, Riemannian Geometry. Oxford Science Publications, The Clarendon Press, Oxford University Press, New York (1993). 